\title{
EXPERIMENTS IN INTERMEDIATE ENERGY PHYSICS
}

\author{
Final Report
}

May 1, 1979 and August 31, 2002

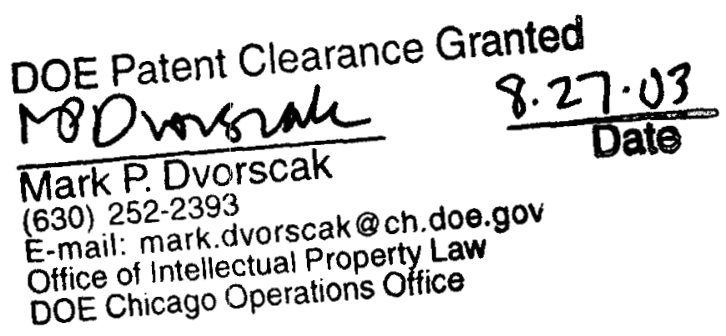

D. Dehnhard

School of Physics \& Astronomy

University of Minnesota

Minneapolis, MN 55455

February 2003 
Disclaimer: Any opinions, findings, and conclusions or recommendations expressed in this report are those of the author and do not necessarily reflect the views of the Department of Energy.

This material is based upon work supported by the U.S. Department of Energy under Award No. DE-AC02-79ER10423 and No. DE-FG02-87ER40362 


\section{DISCLAIMER}

This report was prepared as an account of work sponsored by an agency of the United States Government. Neither the United States Government nor any agency thereof, nor any of their employees, makes any warranty, express or implied, or assumes any legal liability or responsibility for the accuracy, completeness, or usefulness of any information, apparatus, product, or process disclosed, or represents that its use would not infringe privately owned rights. Reference herein to any specific commercial product, process, or service by trade name, trademark, manufacturer, or otherwise does not necessarily constitute or imply its endorsement, recommendation, or favoring by the United States Government or any agency thereof. The views and opinions of authors expressed herein do not necessarily state or reflect those of the United States Government or any agency thereof. 


\section{DISCLAIMER}

Portions of this document may be illegible in electronic image products. Images are produced from the best available original document. 


\section{SUMMARY}

Research in experimental nuclear physics was done from 1979 to 2002 primarily at intermediate energy facilities that provide pion, proton, and kaon beams. Particularly successful has been the work at the Los Alamos Meson Physics Facility (LAMPF) on unraveling the neutron and proton contributions to nuclear ground state and transition densities. This work was clone on a wide variety of nuclei and with great detail on the carbon, oxygen, and helium isotopes. Some of the investigations involved the use of polarized targets which allowed the extraction of information on the spin-dependent part of the $\Delta$-nucleon interaction. At the Indiana University Cyclotron Facility (IUCF) we studied proton-induced charge exchange reactions with results of importance to astrophysics and the nuclear few-body problem. During the first few years, the analysis of heavy-ion nucleus scattering data that had been taken prior to 1979 was completed. During the last few years we created hypernuclei by use of a kaon beam at Brookhaven National Laboratory (BNL) and an electron beam at Jefferson Laboratory (JLab). The data taken at BNL for a study of the non-mesonic weak decay of the $\Lambda$ particle in a nucleus are still under analysis by our collaborators. The work at, JLab resulted in the best resolution hypernuclear spectra measured thus far with magnetic spectrometers. 


\section{Contents}

1 Introduction $\quad 4$

2 Research Goals and Results 5

2.1 Work at LAMPF and IUCF $(1979-1997) \ldots \ldots \ldots$

2.2 Work at BNL and JLab $(1997-2002) \ldots \ldots \ldots \ldots$

3 Publications $r$

3.1 Papers published in refereed journals ................. . . 8

3.2 Papers submitted for publication . . . . . . . . . . . . . 14

3.3 Selected publications in conference proceedings . . . . . . . . . . . 15

3.4 Abstracts submitted to recent meetings (1999-2001) . . . . . . . . 16

3.5 Other publications ........................... 17

4 Advanced degrees granted $\quad 17$

5 Reprints of eleven articles published in Physical Review Letters 19 


\section{Introduction}

This is the final report on the research done under grant numbers DOE/DE-AC02-79ER10423 and DOE/DE-FG02-87ER40362 between the University of Minnesota and the Department of Energy. It covers 23 years of experimental nuclear physics research between 1979 and 2002. The principal investigator of the grants was Prof. D. Dehnhard.

The project title changed over the years:

1979 - 1981 "Nuclear Structure Studies with Pions and Heavy Ions"

1981 - 1984 "Nuclear Structure Studies with Pions and Light and Heavy Ions"

1984 - 1987 "Nuclear Structure Studies with Pions and Light Ions"

1987 - 1997 "Pion- and Proton-Nucleus Interactions at Intermediate Energy"

1997 - 2002 "Experiments in Hypernuclear Physics"

The research was done in collaboration with physicists from many different Universities and National Laboratories. In the last few years, collaborators were mostly from the following institutions: Brookhaven National Laboratory (BNL), Los Alamos National Laboratory (I.ANL), Hampton, Tohoku, and Carnegie Mellon Universities, and the Universities of Houston, Kentucky and Zagreb.

The following postdoctoral research associates contributed in various ways to the research: V. Shkolnik, D. B. Holtkamp, S. J. Seestrom-Morris, S. K. Nanda, M. A. Franey, S. M. Sterbenz, B. Brinkmoeller, M. Palarczyk, J. L. Langenbrunner, J. M. O'Donnell, J. Gerald, H. J. Juengst, and J. H. Liu. Prof. S. Chakravarti of California State University at Pomona provided help with the theoretical analysis of the data during several visits.

Publications in refereed journals, conference proceedings, abstracts submitted to recent meetings and other publications are listed in subsections 3.1-3.5. A list of advanced degrees granted to graduate students and the titles of their Ph.D. or Master of Science (M. S. ) theses is given in subsection 4 .

The research goals and results are discussed in the following section. Reprints of eleven publications in Physical Review Letters are attached as section 5. 


\section{Research Goals and Results}

Over the period of 23 years, experiments in intermediate energy physics were carried out primarily at Los Alamos Meson Physics Facility (LAMPF), the Indiana University Cyclotron. Facility (IUCF), the Brookhaven National Laboratory (BNL) and. most recently, the Jefferson National Accelerator Facility (JLab).

\subsection{Work at LAMPF and IUCF (1979-1997)}

At LAMPF, the main goal of the experiments was the study of nuclear structure using the unique selectivity of the interaction of positive and negative pions with nucleons with respect to the neutron-proton structure of nuclear ground states and of transitions to excited states. Specifically, the studies of elastic and inelastic pion scattering and single-charge exchange reactions on the carbon, oxygen, and helium isotopes provided new knowledge that was not, attainable with conventional nuclear probes such as protons. The pion work was first carried out with unpolarized targets, later with polarized targets. Some of this work was recognized by a report in "Physics News 1979" [1.] which is sponsored by the American Physical Society and presents summaries of important discoveries in a particular year. A summary of our research at LAMPF was also published in the CERN Courier[2].

At IUCF, our work on the $(p, n)$ reaction on the helium isotopes yielded information for: use in astrophysical models of mass accretion of neutron stars as well as on the structure of the mass four system and the quasifree processes that dominate the reactions on helium-3.

\section{Highlights of our research at $\mathrm{LAMPF}$ and IUCF}

- Discovery of nuclear excitations by pions consistent with pure neutron and pure proton transitions. Specifically, the transition to the first $9 / 2^{+}$state in ${ }^{13} \mathrm{C}$ was found to arise only from the promotion of a neutron from the $p_{3 / 2}$ to the $d_{5 / 2}$ shell. PRL43(1979)1091.

- Transitions in the selfconjugate nucleus ${ }^{16} \mathrm{O}$ to three $4^{-}$states showed large $\pi^{+} / \pi^{-}$ asymmetries in inelastic pion scattering. These asymmetries were shown to arise from 
three-state isospin mixing. PRL45(1980)420.

- Transitions involving a spin transfer of 1 were found to have distinctly different incident energy dependences than transitions with a spin transfer of 0. PRL46(1981)1447.

- The interference of proton and neutron transition amplitudes, for example for transitions to $2^{+}$and $4^{-}$states in ${ }^{14} \mathrm{C}$, leads to vanishingly small cross sections for $\pi^{+}$scattering and strongly enhanced cross sections for $\pi^{-}$scattering or vice versa. PRL37(1981)216.

- Only a small amount of isospin mixing was found in pion scattering from ${ }^{4} \mathrm{He}$. This result is in striking contrast to the large amount of isospin mixing inferred from the photonucleon cross section ratio, $\sigma(\gamma, p) / \sigma(\gamma, n)$. PRL57(1986)543.

- Our comprehensive data set for $\pi-{ }^{4}$ He elastic scattering uncovers inconsistencies between existing data sets and allows a determination of $\pi-{ }^{4}$ He phase shifts. PRC4419912031.

- $\pi^{+} / \pi^{-}$cross section ratios for proton and triton knockout reactions on ${ }^{4} \mathrm{He}$ cover a large range of values between 0.3 and about 50 in contrast to the expected values between 6 and 8. The results are interpreted as due to interference effects. PRC46199252 and PRL6919941508.

- First measurement of neutron spectra from the ${ }^{4} \mathrm{He}(\mathrm{p}, \mathrm{n})^{4} \mathrm{Li}$ reaction at intermediate energies resolve the controversy over the existence of narrow structures in ${ }^{4} \mathrm{Li}$. This experiment provides the data needed by current astrophysical models of solar flares and of the mass accretion of neutron stars. PLB369(1996)39.

- First measurement of asymmetries in pion scattering and pion-induced single charge exchange on polarized ${ }^{3} \mathrm{He}$. Evidence is found for the importance of the $\Delta$-neutron spin-spin interaction. PRL76(1996)3667, PRC56(1997)2607, and PRC60(1999)24001.

\subsection{Work at BNL and JLab (1997-2002)}

At BNL, we created hypernuclei using the kaon beam of the Alternating Gradient Synchroton (AGS). Specifically, we studied the formation of the hypernucleus ${ }_{\Lambda}^{12} \mathrm{~B}$ using the $\left(\mathrm{K}^{-}, \pi^{0}\right)$ 
reaction on ${ }^{12} \mathrm{C}$ (BNL experiment E907). The $\pi^{0}$ mesons were detected with the Neutral Meson Spectrometer (NMS). The analysis of E907 has been completed and a paper has been submitted for publication to Physical Review C.

In addition, we took data for a study of the nucleon-induced, non-mesonic weak decay of the $\Lambda$ in the ${ }_{\Lambda}^{4} \mathrm{H}$ hypernucleus (BNL experiment E931). The formation of a ${ }_{\Lambda}^{4} \mathrm{H}$ by the $\left(\mathrm{K}^{-}\right.$, $\pi^{0}$ ) reaction on a liquid ${ }^{4} \mathrm{He}$ target was identified by the detection of the $\pi^{0}$ mesons with the NMS. The neutrons and protons from the decay of the residual hypernucleus ${ }_{\Lambda}^{4} \mathrm{H}$ were detected by large arrays of proton and neutron detectors.

The preparation and execution of E931 has been our major effort during the past 16 months. Postdoc Henry Juengst (HGJ) supervised the work on all aspects of the experiment, spending long time periods at BNL. Postdoc Jinghua Liu (JHL) performed calculations of simulations of beam and reaction particle detection devices that proved important. in the planning of the experiments. During the last two beam periods all components of the very complex setup were working properly. These include numerous beam detection devices, the neutral meson spectrometer (NMS), arrays of neutron detectors and sets of timing scintillators and proton tracking chambers to detect the neutrons and protons from the nucleon-induced decay of the $\Lambda$, and, most importantly, the liquid helium target.

The responsibility of the analysis of the data has been taken on by the participants from Carnegie Mellon University (CMU). It will be the basis of a Ph.D. thesis of a CMU student. At the time of this report it is not possible yet to determine whether this effort has produced a result of the required accuracy.

In addition, we worked on the analysis of the high-resolution study of the electroproduction of ${ }_{\Lambda}^{12} \mathrm{~B}$ using the ${ }^{12} \mathrm{C}\left(\mathrm{e}, \mathrm{e}^{\prime} \mathrm{K}^{+}\right)$reaction at JLab (Experiment E89-009). JHL had been. instrumental in the writing of the computer codes for taking and analyzing the data.

Due to the full-time commitment of HGJ to E931, no progress was made during the last 16 months on the analysis of existing data taken with the CEBAF Large Acceptance Spectrometer (CLAS) (E89-004). However, this work is currently being continued by HG.J 
who is now a postdoctoral research associate at George Washington University.

Finally, we mention that during the first three years of the 23-year period, part of the effort was directed to completing the analysis of experimental data on heavy-ion induced reactions and scattering that had been taken with the MP Tandem accelerator at the University of Minnesota prior to 1979. In particular we cite the optical model study of elastic scattering of ${ }^{16} \mathrm{O}$ from ${ }^{28} \mathrm{Si}$ at many different incident energies. PRC28(1983)294.

\section{References}

[1] "The Roles of Neutrons and Protons in Nuclear Excitations,"

Physics News in 1979, edited by Phillip Schewe for the American Institute of Physics, p. 65 (1979).

[2] "Los Alamos, Nuclear Spectroscopy with Pions," CERN Courier 22, 13 (1982), edited by B. Southworth, G. Fraser, and H.-L. Felder.

\section{Publications}

\subsection{Papers published in refereed journals}

1. "Pure Neutron and Pure Proton Transitions in Inelastic Scattering of Pions by ${ }^{13} \mathrm{C}, "$ D. Dehnhard, S. J. Tripp, M. A. Franey, G. S. Kyle, C. L. Morris, R. L. Boudrie, J. Piffaretti and H. A. Thiessen, Phys. Rev. Lett. 43, 1091 (1979).

2. "Isospin Mixing of 4- Particle-Hole States in ${ }^{16} \mathrm{O}, "$ D. B. Holtkamp, W. J. Braithwaite, W. B. Cottingame, S. J. Greene, R. J. Joseph, C. F. Moore, C. L. Morris, J. Piffaretti, E. R. Siciliano, H. A. Thiessen and D. Dehnhard, Phys. Rev. Lett. 45, 420 (1980).

3. "Two-Proton Transfer Reaction ${ }^{48} \mathrm{Ca}\left({ }^{18} \mathrm{O},{ }^{16} \mathrm{C}\right){ }^{50} \mathrm{Ti}$ at $102 \mathrm{MeV}$;" D. Dehnhard, W. Saathoff, T. S. Bhatia, K. Wannebo, and C. A. Wiedner, Z. Physik 291, 71 (1979). 
4. "The j-Dependence of the $\left({ }^{19} \mathrm{~F},{ }^{16} \mathrm{O}\right)$ Reactions," S. Kubono, D. A. Lewis, and D. Dehnhard, Nucl. Phys. A334, 336 (1980).

5. "Identification of $\Delta S=1$ Transitions in ${ }^{13} \mathrm{C}$ by Measurement of Pion Inelastic Excitation Functions," S. J. Seestrom-Morris, D. Dehnhard, D. B. Holtkamp, and C. L. Morris, Phys. Rev. Lett. 46, 1447 (1981).

6. "Strong Cancellations of Neutron and Proton Transition Amplitudes Observed in Pion Inelastic Scattering from ${ }^{14}$ C," D. B. Holtkamp, S. J. Seestrom-Morris, S. Chakravarti, D. Dehnhard, H. W. Baer, C. L. Morris, S. J. Greene, and C. J. Harvey, Phys. Rev. Lett. 47, $216(1981)$.

7. "Elastic and Inelastic Scattering of $0.8 \mathrm{GeV}$ Protons from ${ }^{24} \mathrm{Mg}$ and ${ }^{26} \mathrm{Mg}$," G. S. Blanpied, N. M. Hintz, G. S. Kyle, M. A. Franey, S. J. Seestrom-Morris, R. K. Owen, J. W. Palm, D. Dehnhard, M. L. Barlett, C. J. Harvey, G. W. Hoffmann, J. A. McGill, R. P. Liljestrand, and L. Ray, Phys. Rev. C 25, 422 (1982).

8. "Inelastic Scattering of Pions from ${ }^{13} \mathrm{C}$ at $162 \mathrm{MeV}$, S. J. Seestrom-Morris, D. Dehnhard, M. A. Franey, G. S. Kyle, C. L. Morris, R. L. Boudrie, H. A. Thiessen, and J. Piffaretti, Phys. Rev. C 26, 954 (1982).

9. "Elastic Scattering of ${ }^{16} \mathrm{O}$ by ${ }^{28} \mathrm{Si}$, V. Shkolnik, D. Dehnhard, and M. A. Franey, Phys. Rev. C 28, 294 (1983).

10. "Collective Model Analysis of Pion Scattering from ${ }^{13}$ C," S. J. Seestrom-Morris, D. Dehnhard, M. A. Franey, G. S. Kyle, C. L. Morris, R. L. Boudrie, H. A. Thiessen, and J. Piffaretti, Phys. Rev. C 28, 1301 (1983).

11. "Measurement of the ${ }^{12} \mathrm{C}\left(\pi, \pi^{\prime} \gamma\right){ }^{12} \mathrm{C}\left(2^{+}, \mathrm{T}=0 ; 4.44 \mathrm{MeV}\right)$ Angular Correlations: A Test of the Isobar-Hole Model," F. Vogler, R. Olszewskli, M. Meyer, E. L. Mathie, G. R. Smith, E. Boschitz, S. Chakravarti, D. Dehnhard, and M. Thies, Phys. Lett. 134B, 161 (1984). 
12. "The ${ }^{13} \mathrm{C}\left(\vec{p}, p^{\prime}\right){ }^{13} \mathrm{C}$ Reaction at $\mathrm{T}_{p}=547 \mathrm{MeV}$ " S. J. Seestrom-Morris, M. A. Franey, D. Dehnhard, D. B. Holtkamp, R. L. Boudrie, J. F. Amann, G. C. Idzorek, and C. A. Goulding, Phys. Rev. C 30, 270 (1984).

13. "Excitation of the $1^{+}$State in ${ }^{48} \mathrm{Ca}(10.2 \mathrm{MeV})$ by Inelastic Scattering of $\pi^{+}$and $\pi^{-}, " \mathrm{D}$. Dehnhard, D. H. Gay, C. L. Blilie, S. J. Seestrom-Morris, M. A. Franey, C. L. Morris, R. L. Boudrie, T. S. Bhatia, C. Fred Moore, L. C. Bland, and H. Ohnuma, Phys. Rev. C 30, 242 (1984).

14. "Small Angle Pion Inelastic Scattering from ${ }^{12} \mathrm{C}$ at $162 \mathrm{MeV}$," C. L. Morris, N. Tanaka, R. L. Boudrie, L. C. Bland, H. T. Fortune, R. Gilman, S. J. Seestrom-Morris, C. Fred Moore, D. Dehnhard, Phys. Rev. C 30, 662 (1984).

15. "Isospin Structure of Transitions in ${ }^{17} \mathrm{O}$ from Inelastic Pion Scattering at $164 \mathrm{MeV}$," C. L. Blilie, D. Dehnhard, M. A. Franey, D. H. Gay, D. B. Holtkamp, S. J. SeestromMorris, P. J. Ellis, C. L. Morris, and D. J. Millener, Phys. Rev. C 30, 1989 (1984).

16. "Pion Scattering to $4^{-}$States in ${ }^{14}$ C," D. B. Holtkamp, S. J. Seestrom-Morris, D. Dehnhard, H. W. Baer, C. L. Morris, S. J. Greene, C. J. Harvey, D. Kurath, and J. A. Carr, Phys. Rev. C 31, 957 (1985).

17. "M4 Transitions Observed in Pion Inelastic Scattering on ${ }^{15}$ N," S. J. Seestrom-Morris, D. Dehnhard, C. L. Morris, L. C. Bland, R. Gilman, H. T. Fortune, D. J. Millener, D. P. Saunders, P. A. Seidl, Rex. R. Kiziah, and C. Fred Moore, Phys. Rev. C 31, 923 (1985).

18. "Large-angle elastic scattering of $\pi^{+}$and $\pi^{-}$from ${ }^{16} \mathrm{O}$ at $114 \mathrm{MeV}$," Kalvir S. Dhuga, G. R. Burleson, J. A. Faucett, R. L. Boudrie, W. B. Cottingame, S. J. Greene, C. L. Morris, N. Tanaka, Z. F. Wang, S. Nanda, D. Dehnhard, J. D. Zumbro, S. Mordechai, C. Fred Moore, and D. J. Ernst, Phys. Rev. C 32; R2208 (1985).

19. "Elastic $\pi^{+}$and $\pi^{-}$Scattering on ${ }^{14} \mathrm{C}$ at $164 \mathrm{MeV}$," Carol J. Harvey, H. W. Baer, J. A. Johnstone, C. L. Morris, S. J. Seestrom-Morris, D. Dehnhard, D. B. Holtkamp, S. J. Greene, Phys. Rev. C 33, 1454 (1986). 
20. "A Comparative Study of the ${ }^{13} \mathrm{C}(\mathrm{p}, \mathrm{p} /)^{13} \mathrm{C}$ and ${ }^{13} \mathrm{C}(\mathrm{p}, \mathrm{n}){ }^{13} \mathrm{~N}$ Reactions at $\mathrm{E}_{p}=35$ MeV," H. Ohnuma, B. A. Brown, D. Dehnhard, K. Furulawa, T. Hasegawa, S. Hayakawa, N. Hoshino, K. Ieki, M. Kabasawa, K. Maeda, K. Miura, K. Muto, T. Naliagawa, K. Nisimura, H. Orihara, T. Suehiro, T. Tohei, and M. Yasue, Nucl. Phys. A 456, 61 (1986).

21. "Measurement of Isospin Mixing in ${ }^{4} \mathrm{He}$ and its Implications for Charge Symmetry Breaking," C. L. Blilie, D. Dehnhard, D. B. Holtkamp, S. J. Seestrom-Morris, S. K. Nanda, W. B. Cottingame, D. Halderson, C. L. Morris, C. Fred Moore, P. A. Seidl, H. Ohnuma, and K. Maeda, Phys. Rev. Lett. 57, 543 (1986).

22. "Test of the $\Delta$-Hole Model through the Energy Dependence of $\left(\pi, \pi^{\prime} \gamma\right)$ Angular Correlations on ${ }^{12} \mathrm{C}^{\prime \prime}$, R. Olszewski, R. Baran, A. Hoffmann, S. Krell, H. W. Ortner, J. Orzechowski, G. Schmidtlein, F. Vogler, G. R. Smith, W. List, D. Dehnhard, and M. Thies, Phys. Rev. Lett. 57, 2143 (1986).

23. "A Large-Angle Pion-Nucleus Scattering Facility for EPICS at LAMPF", G. R. Burleson, W. V. Cottingame, K. S. Dhuga, J. A. Faucett, C. P. Fontenla, J. F. Amann, R. L. Boudrie, S. J. Greene, C. L. Morris, N. Tanaka, Z. F. Wang, D. Yusnukis, M. Brown, R. R. Kiziah, E. C. Milner, C. F. Moore, S. Mordechai, D. Oakley, P. A. Seidl, C. L. Blilie, D. Dehnhard, S. Nanda, S. J. Seestron-Morris, J. D. Zumbro, and K. Maeda, Nucl. Instr. and Meth. A247, 327 (1986).

24. "Distorted-wave impulse approximation and coupled-channels analysis of inelastic pion scattering from ${ }^{18}$ O", S. Chakravarti, D. Dehnhard, M. A. Franey, S. J. SeestromMorris, D. B. Holtkamp, C. L. Blilie, A. C. Hayes, C. L. Morris, and D. J. Millener, Phys. Rev. C 35, 2197 (1987).

25. "Neutron and proton matrix elements for $2_{1}^{+}$transitions in $\mathrm{T}=1$ nuclei from pion inelastic scattering", C. L. Morris, S. J. Seestrom-Morris, D. Dehnhard, C. L. Blilie, R. Gilman, G. P. Gilfoyle, J. D. Zumbro, M. G. Burlein, S. Mordechai, H. T. Furtune, L. C. Bland, M. Brown, D. P. Saunders, P. A. Seidl, C. Fred Moore, K. Maeda, G. S. Blanpied, and B. A. Brown, Phys. Rev. C 35, 1388 (1987). 
26. "Structure of the low-lying $2^{+}$states in ${ }^{14} \mathrm{C}$ from inelastic pion scattering", S. Chakravarti, D. Dehnhard, P. J. Ellis, D. B. Holtkamp, Li-Ping Lung, S. J. Seestrom-Morris, H. Baer, C. L. Morris, S. J. Greene, and C. J. Harvey, Phys. Rev. C 37, 1554 (1988).

27. "Pion inelastic scattering to the three lowest $2^{+}$states of ${ }^{18} \mathrm{O}$ ", S. J. Seestrom-Morris, D. Dehnhard, D. B. Holtkamp, C. L. Blilie, C. L. Morris, J. D. Zumbro, and H. T. Fortune, Phys. Rev. C 37, 2057 (1988).

28. "Excitation function of the $15.11 \mathrm{MeV}\left(1^{+}, \mathrm{T}=0\right)$ state in ${ }^{12} \mathrm{C}$ from $\left(\pi, \pi^{\prime} \gamma\right)$ angular correlations", R. Baran, J. Götz, A. Hofmann, D. Malz, R. Müller, R. Olszewski, H. W. Ortner, and D. Dehnhard, Phys. Rev. C 37, 2892 (1988).

29. "Medium effects in $\left(\pi, \pi^{\prime} \gamma\right)$ angular correlations on ${ }^{12} \mathrm{C}\left(2^{+}\right)$", R. Olszewski, R. Baran, A. Hofmann, S. Krell, H. W. Ortner, J. Orzechowsky, G. Schmidtlein, F. Vogler, W. List, G. R. Smith, D. Dehnhard, M. Thies, Phys. Rev. C 37, 2665 (1988).

30. "12 $\mathrm{C}\left(\pi^{ \pm}, \pi^{ \pm^{\prime}}, \mathrm{p}\right)^{11} \mathrm{~B}$ reaction near the giant dipole region", Sung Hoon Yoo, A. Williams, S. Mordechai, C. Fred Moore, C. L. Morris, S. J. Seestrom-Morris, M. K. Jones, S. M. Sterbenz, D. Dehnhard, D. S. Oakley, A. Fazely, Phys. Rev. Lett. 63, 738 (1989).

31. "The ${ }^{16} \mathrm{O}\left(\overrightarrow{\mathrm{p}}, \mathrm{p}^{\prime}\right)$ reaction at $35 \mathrm{MeV}$ ", H. Ohnuma, N. Hoshino, K. Ieki, M. Iwase, H. Shimizu, H. Toyokawa, T. Hasegawa, K. Nisimura, M. Yasue, H. Kabasawa, T. Nakagawa, T. Tohei, H. Orihara, S. I. Hayakawa, K. Miura and T. Suehiro, S. K. Nanda, D. Dehnhard, and M. A. Franey, Nucl. Phys. A514, 273 (1990).

32. "He $\left(\pi, \pi^{\prime} p\right)^{3} \mathrm{H}$ reaction: quasi-free and resonance scattering," M. K. Jones et al., Phys. Rev. C 42, R807 (1990).

33. "208 $\mathrm{Pb}\left(\pi^{ \pm}, \pi^{ \pm^{\prime}} \mathrm{n}\right)$ reaction near the giant Resonance region," Sung Hoon Yoo et al., J. Phys. G: Nucl. Part. Phys. 16, L89 (1990).

34. "Analyzing powers for the reaction $\pi^{-} \vec{p} \rightarrow \pi^{\circ} n$ at $T_{\pi^{-}}=161 \mathrm{MeV}$," J. J. Görgen et al., Phys. Rev. D 42, 2374 (1990). 
35. "Pion elastic scattering from polarized ${ }^{13} \mathrm{C}$ in the energy region of the $[3,3]$ resonance," Yi-Fen Yen et al., Phys. Rev. Lett. 66, 1959 (1991).

36. "Analyzing powers for pion charge exchange on polarized ${ }^{13} \mathrm{C}$," J. J. Görgen et al., Phys. Rev. Lett. 66, 2193 (1991).

37. "Elastic scattering of $\pi^{+}$and $\pi^{-}$from ${ }^{4} \mathrm{He}$ between 90 and $240 \mathrm{MeV}, \mathrm{B}$. Brinkmöller et al., Phys. Rev. C 44, 2031 (1991).

38. "Inelastic scattering of polarized protons from ${ }^{4}$ He at 500 and $800 \mathrm{MeV}$," S. M. Sterbenz et al., Phys. Rev. C 45, 2578 (1992).

39. " $\mathrm{He}\left(\pi, \pi^{\prime} \mathrm{p}\right)^{3} \mathrm{H}$ reaction near the $\mathrm{P}_{33} \pi$-nucleon resonance," M. K. Jones et al., Phys. Rev. C 46, 52 (1992).

40. "Evidence for direct triton knockout in the ${ }^{4} \mathrm{He}(\pi, \pi t)$ reaction," J. L. Langenbrunner et al., Phys. Rev. Lett. 69, 1508 (1992).

41. "Pion elastic scattering from polarized ${ }^{13} \mathrm{C}$ in the energy region of the $P_{33}$ resonance," Yi-Fen Yen et al., Phys. Rev. C 50, 897 (1994).

42. "Study of the isospin dependence of the ${ }^{4} \mathrm{He}$ continuum using the ${ }^{4} \mathrm{He}(\mathrm{p}, \mathrm{p}$ 'X) reaction," B. A. Raue et al., Phys. Rev. C 52, R445 (1995).

43. "Asymmetries for elastic scattering of $\pi^{+}$from polarized ${ }^{3} \mathrm{He}$ and the $\Delta$-neutron spinspin interaction," M. A. Espy et al., Phys. Rev. Letters 76, 3667 (1996).

44. "The ${ }^{4} \mathrm{He}(\vec{p}, \mathrm{n})^{4} \mathrm{Li}^{*}$ reaction at 100 and $200 \mathrm{MeV}$ : Implications to the mass-four system and to astrophysics," C. M. Edwards et al., Phys. Lett. B 368, 39 (1996).

45. "Asymmetries for elastic scattering of $\pi^{ \pm}$from polarized ${ }^{3} \mathrm{He}$ at $\Delta$ resonance energies," M. A. Espy et al., Phys. Rev. C 56, 2607 (1997).

46. "Cross sections and analyzing powers for the $(\vec{p}, n)$ reaction on ${ }^{3} \mathrm{He}$ and ${ }^{4} \mathrm{He}$ at 200 MeV," M. Palarczyk, C. M. Riedel, D. Dehnhard, M. A. Espy, M. A. Franey, J. L. 
Langenbrunner, L. C. Bland, D. S. Carman, B. Brinkmöller, R. Madey, Y. Wang, and J. W. Watson, Phys. Rev. C 58, 645 (1998).

47. "Zero degree differential cross sections and $D_{N N}$ values for the ${ }^{17,18} O(\vec{p}, \mathrm{n})^{17,18 \mathrm{~F}}$ reactions at $E_{p}=118 \mathrm{MeV}, "$ I. J. van Heerden, M. Palarczyl, X. Wang, J. Rapaport, D. L. Prout, C. D. Goodman, B. A. Luther, E. Sugarbaker, T. N. Taddeucci, C. C. Foster, K. Hicks, L. Wang, X. Yang, D. Dehnhard, J. M. O'Donnell, H. Ohnuma, B. K. Park, G. R. Burleson, S. Blanchard, Phys. Rev. C 59, 1488 (1999).

48. "Measurements of asymmetries of pion single charge exchange on polarized ${ }^{3} \mathrm{He}$ at 200 MeV," Q. Zhao, G. Burleson, S. Blanchard, T. Chang, W. Gibbs, J. Haas, B. Park, M. Whitton, M. Espy, D. Dehnhard, B. Larson, J. O'Donnell, M. Palarczyk, W. Cummings, P. Delheij, O. Häusser, E. Pasyuk, M. Gostkin, J. Amann, R. Boudrie, C. Riedel, C. Morris, S. Pentillä, D. Swenson, D. Tupa, J. Comfort, C. Gaulard, K. Maeda, G. Glass, I. Supek, Phys. Rev. C 60, 24001 (1999).

49. "The construction and operating characteristics of a cathode strip chamber system designed to measure the reaction vertices of a stopping kaon beam," M. W. Ahmed, D. Androic, I. Bertovic, J. Bjoraker, R. Chrien, X. Cui, D. Dehnhard, A. Empl, M. Furic, J. Gerald, R. Gill, E. V. Hungerford, H. Juengst, K. J. Lan, J. H. Liu, C. L. Morris, J. M. O’Donnell, J. C. Peng, T. Petkovic, P. Pile, M. Planinic, C. M. Riedel, A. Rusek, R. Sutter, L. Tang, H. A. Thiessen, M. Youn, V. Zeps, Nucl. Instr. Meth. A 469, 95 (2001).

\subsection{Papers submitted for publication}

1. "Experimental Study of the ${ }^{12} \mathrm{C}\left(\mathrm{K}_{\text {stopped }}, \pi^{0}\right)^{12 \wedge} \mathrm{B}$ Reaction," M. W. Ahmed, X. Cui, A. Empl, E. V. Hungerford, K. J. Lan, M. Youn, R. E. Chrien, R. Gill, P. Pile, A. Rusek, R. Sutter, J. Bjoraker, D. Dehnhard, J. M. O'Donnell, J. Gerald, H. Juengst, J. H. Liu, J. C. Peng, C. L. Morris, C. M. Riedel, H. A. Thiessen, D. Androic, I. Bertovic, M. Furic, T. Petkovic, M. Planinic, L. Tang, V. Zeps, R. J. Peterson, submitted for publication in Phys. Rev. C (2002). 
2. "Energy Calibration of the CsI Crystals of the Neutral Meson Spectrometer (NMS) using Monoenergetic $\pi^{0}$ from the Decay of Stopped $K^{+}, " H$. G. Juengst, M. Ahmed, D. Androic, R. Barber, I. Bertovic, J. Bjoraker, R. Chrien, D. Dehnhard, M. Furic, R. Gill, E. Hungerford, J. H. Liu, J. O’Donnell,J. Peng, P. Pile, C. M. Riedel, A. Rusek, M. Sarsour, R. Sutter, T. Vidnovic, M. Youn, V. Zeps, submitted for publication in Nuclear Instruments and Methods, 2002.

3. "High Resolution Spectroscopy of the ${ }_{\Lambda}^{12} \mathrm{~B}$ Hypernucleus Produced by the $\left(\mathrm{e}, \mathrm{e}^{\prime} \mathrm{K}^{+}\right)$Reaction," T. Miyoshi, (The HNSS Collaboration, 69 members), submitted for publication in Physical Review Letters, Jan. 2003.

\subsection{Selected publications in conference proceedings}

1. "Pion-Nucleus Interactions (Experiments)," D. Dehnhard, Proceedings Ninth International Conference on High Energy Physics and Nuclear Structure, Versailles, France, July 6-10, 1981, Nucl. Phys. A374, 377c (1982).

2. "Nuclear Structure Studies Using Pion Inelastic Scattering,"

D. Dehnhard and S. J. Seestrom-Morris (presented by D. D.), Proceedings International Symposium on Electromagnetic Properties of Atomic Nuclei, Tokyo, Japan, Nov. 1983, edited by H. Horie and H. Ohnuma, Tokyo Institute of Technology, p. 225 (1984).

3. "Anomalous Spin Response in the ${ }^{4} \mathrm{He}\left(\overrightarrow{\mathrm{p}}, \overrightarrow{\mathrm{p}}^{\prime}\right)^{4} \mathrm{He} \mathrm{e}^{*}$ Reaction at $500 \mathrm{MeV}$ ", S. K. Nanda, S. M. Sterbenz, D. Dehnhard, M. K. Jones, C. E. Parman, Yi-Fen Yen, and K. W. Jones, Proceedings 8th International Symposium on High Energy Spin Physics, Minneapolis, MN Sept. 6-18, 1988, ed. by K. J. Heller. AIP Conf. Proc. No. 187, p. 681, N. Y. (1989).

4. "High Energy Kaon and Pion Scattering," B. Brinkmöller, D. Dehnhard, and S. Chakravarti, Proceedings Workshop on Science at the KAON Factory, TRIUMF, Vancouver, B. C., Canada, July 23-28, 1990. 
5. "Pion Elastic Scattering on a Polarized ${ }^{13} \mathrm{C}$ Target," D. Dehnhard, Yi-Fen Yen, M. A. Franey, and S. Chakravarti (Presented by D. D.), Proceedings International Symposium on Mesons and Light Nuclei V, Prague, C.S.F.R., September 1-6, 1991, Few-Body Systems, Suppl. 5, 274 (1992).

6. "New results on spin effects in pion-nucleus scattering and single-charge exchange," D. Dehnhard, Proceedings of Meson'96 Workshop on Production, Properties and Interaction of Mesons, Cracow, Poland, May 10-14, 1996, Acta Physica Polonica B 27, $3019(1996)$.

\subsection{Abstracts submitted to recent meetings (1999 - 2001)}

1. "Energy Calibration of the CsI Crystals of the Neutral Meson Spectrometer (NMS) Using the Monoenergetic $\pi^{0}$ from the Decay of stopped $\mathrm{K}^{+}$," Henry Juengst on behalf of the E907 and E931 collaborations at BNL. 1999 Spring Meeting in Atlanta, paper VB04.11, Instrumentation for Nuclear Physics II.

2. "Recent Results from the Study of ${ }_{\Lambda}^{12} \mathrm{~B}$ Levels Using the $\left(\mathrm{K}_{\text {stopped }}^{-}, \pi^{0}\right.$ ) Reaction on ${ }^{12} \mathrm{C}$," Mohammad Ahmed on behalf of the E907 collaboration. 1999 Spring Meeting in Atlanta, paper IB04.08, Sub-Nucleonic Degrees of Freedom.

3. Electroproduction of hypernuclei at Jlab, Liguang Tang, Hampton University, E89-009 Collaboration, BAPS 45, No. 5, page 59, paper ED-2. (DNP 2000 Fall meeting).

4. "Spectroscopic study of electroproduced light $\Lambda$ hypernuclei," Y. Sato, Hampton University, E89-009 Collaboration, BAPS 45, No. 5, page 87, paper KD-10. (DNP Fall 2000 meeting).

5. "Hypernuclear spectroscopic study at TJNAF," Jinghua Liu, University of Minnesota, E89-009 Collaboration, BAPS 46 (2001), No. 2, page 118, paper Q8-5. (APS April 2001 meeting).

6. "Photoproduction of hyperon resonances," Henry G. Juengst, CLAS Collaboration, BAPS 46 (2001), No. 2, page 118, paper Q8-1. (APS April 2001 meeting). 
7. "Photoproduction of Hyperon Resonances on Hydrogen," Henry G. Juengst, University of Minnesota, CLAS Collaboration. Abstract submitted to the International Conference on Nuclear Physics, Berkeley, CA, July 30 to August 3, 2001.

\subsection{Other publications}

1. "Pion-nucleus interactions with high-energy pions", D. J. Ernst and D. Dehnhard, in PILAC Users Group Report on the Physics with PILAC, Los Alamos National Laboratory Report, LA-UR-92-150, 161 (1991).

2. "Pion probes", contribution to "Research at LAMPF", February 1992, prepared for presentation to the NSAC committee.

\section{Advanced degrees granted}

1. Susan J. Seestrom-Morris, Ph.D., Sept. 1981.

Title of Ph.D. Thesis:

"The structure of ${ }^{13} \mathrm{C}$ studied by pion scattering near the [3,3] resonance."

2. M. Garakani, M. S., June 1984.

Title of Master's Plan B paper:

"Pion scattering to the three lowest $2^{+}$states in ${ }^{14} \mathrm{C} . "$

3. Charles L. Blilie, Ph. D., February 1985.

Title of Ph. D. Thesis:

"Pion scattering from ${ }^{4} \mathrm{He} . "$

4. Li-Ping Lung, M. S., July 1986.

Title of Master's Plan B paper:

"Analysis of elastic and inelastic pion scattering from ${ }^{48} \mathrm{Ca} . "$ 
5. C. E. Parman, M.S., December 1988

Title of Master's Plan B paper:

"The reaction ${ }^{4} \mathrm{He}\left(\mathrm{p}, \mathrm{p}^{\prime}\right)^{4} \mathrm{He}$ at $\mathrm{T}_{p}=800 \mathrm{MeV}$ and analysis of the $0^{+}, 20.1 \mathrm{MeV}$ excitation".

6. Mark K. Jones, Ph.D., May 1989

Title of Ph.D. Thesis:

"Pion elastic scattering and the $(\pi, \pi \mathrm{p})$ reaction on ${ }^{4} \mathrm{He}$ in the $\Delta$ region."

7. Yi-Fen Yen, Ph. D., December 1991.

Title of Ph. D. Thesis:

"Pion elastic scattering from polarized ${ }^{13} \mathrm{C}$ in the energy region of the $\mathrm{P}_{33}$ pion-nucleon resonance."

8. Yi-Ju Yu, M. S., January 1993.

Title of Master's Plan B paper:

"Ray trace calculations for pion scattering experiments from polarized ${ }^{13} \mathrm{C}$."

9. Peter Yurek, M. S., August 1993.

Title of Master's Plan B paper:

"Feasibility of a measurement of asymmetries of the pion-induced single charge exchange reaction on polarized ${ }^{3} \mathrm{He} . "$

10. Carla M. Edwards (Riedel), Ph. D., 1995.

Title of Ph. D. Thesis:

"Proton-induced charge exchange on Helium-4 at intermediate energies."

11. Michelle A. Espy, Ph. D., 1996.

Title of Ph. D. Thesis:

"Asymmetries for elastic scattering of charged pions from polarized Helium-3 at Delta resonance energies" 
12. Brian Davis, M. S., 1996.

Title of Master's plan B paper:

"The $\left(K_{\text {stop }}^{-}, \pi^{0}\right)$ reaction: A new tool in hypernuclear physics."

13. Jefferson Bjoraker, M.S. 1998.

Title of Master's plan B paper:

"Contributions to hypernuclear experiments at Brookhaven National Laboratory." 
5 Reprints of eleven articles published in Physical Review Letters 


\title{
Pure Neutron and Pure Proton Transitions in Inelastic Scattering of Pions by ${ }^{13} \mathrm{C}$
}

\author{
D. Dehnhard, S. J. Tripp, M. A. Franey, and G. S. Kyle \\ University of Minnesota, Minneapolis, Minnesota 55455 ; \\ and \\ C. L. Morris, R. L. Boudrie, J. Piffaretti, and H. A. Thiessen \\ Los Alamos Scientific Laboratory, Los Alamos, New Mexico 87545 \\ (Received 18 July 1979)

\begin{abstract}
Differential cross sections $\sigma_{\pi^{ \pm}}(\theta)$ for elastic and inelastic scattering of $162-\mathrm{MeV}$ pions from ${ }^{13} \mathrm{C}$ were measured at momentum transfers between 0.6 and $2.1 \hbar \cdot \mathrm{fm}^{-1}$. For two inelastic transitions, enhancement of $\sigma_{\eta}$ - over $\sigma_{\pi}+$ and of $\sigma_{\pi^{+}}$over $\sigma_{\pi}$ - were observed which are consistent with the values for pion scattering from free nucleons. The results are discussed qualitatively in terms of simple shell-model and weak-coupling-model configurations.
\end{abstract}

In nuclear structure physics, high-resolution and high-intensity intermediate-energy pion beams are presently being employed with increasing success. For example, recent comparative studies ${ }^{1-4}$ of $\pi^{+}$and $\pi^{-}$scattering at pion energies near the $[3,3]$ resonance yielded new information on differences between neutron and proton groundstate and transition densities. For inelastic scattering from nuclei with a neutron excess, measured $^{2-4}$ cross-section ratios $R=\sigma_{\pi-}(\theta) / \sigma_{\pi}+(\theta)$ range from 0.8 to 1.9. These values are not as different from unity as the ratios for pion scattering from free nucleons: $R \simeq \frac{1}{9}$ for protons and $R \simeq 9$ for neutrons at the $[3,3]$ resonance. This result is not unexpected for the collectively enhanced transitions in even- $A$ nuclei on which current studies have concentrated. ${ }^{2-4}$ Results closer to the free-nucleon values may be obtained for odd-A nuclei in transitions between single-particle states or between states which differ only by a particle-hole excitation. The ${ }^{13} \mathrm{C}$ nucleus appears particularly well suited for such a study since it shows single-particle and collective features in a number of low-lying levels. ${ }^{5}$

We have therefore measured differential cross sections for elastic and inelastic scattering of $\pi^{+}$ and $\pi^{-}$from ${ }^{13} \mathrm{C}$ at $162 \mathrm{MeV}$ using the EPICS syste $\mathrm{m}^{6}$ at the Clinton P. Anderson Meson Physics Facility (LAMPF). The target ${ }^{7}$ was a $15-\mathrm{cm} \times 23$ $\mathrm{cm}$ sheet of carbon, $210 \mathrm{mg} / \mathrm{cm}^{2}$ thick, enriched in ${ }^{13} \mathrm{C}$ to $99 \%$, and covered on both sides by thin $\left(\sim 1 \mathrm{mg} / \mathrm{cm}^{2}\right)$ Kapton foils. The energy resoluticn for all scattering angles was better than $300 \mathrm{keV}$ (full width at half maximum).

The most striking result of this experiment is the very large value, $R \sim 9$, for the transition to a state at $9.5 \mathrm{MeV}$. This result is consistent with the free $\pi$-neutron value of 9 . At angles $\theta_{\text {c.m. }}$. $\geqslant 50^{\circ}$ and $T_{\pi}=162 \mathrm{MeV}$ (momentum transfers $\geqslant 1 \hbar \cdot \mathrm{fm}^{-1}$ ) we find this state quite strongly excited by $\pi^{-}$but so weak in $\pi^{+}$scattering that it is often difficult to discriminate from the background (Fig. 1). We also observe a significant enhancement of $\pi^{+}$scattering over $\pi^{-}$scattering for a group of states at about $16 \mathrm{MeV}$ excitation. Around $21 \mathrm{MeV}$ there are differences in the structure of the $\pi^{+}$and $\pi^{-}$spectra [Figs. 1(a) and 1(b)] quite similar to results of a recent experiment ${ }^{8}$ on ${ }^{12} \mathrm{C}$. The centroid of this group of states is

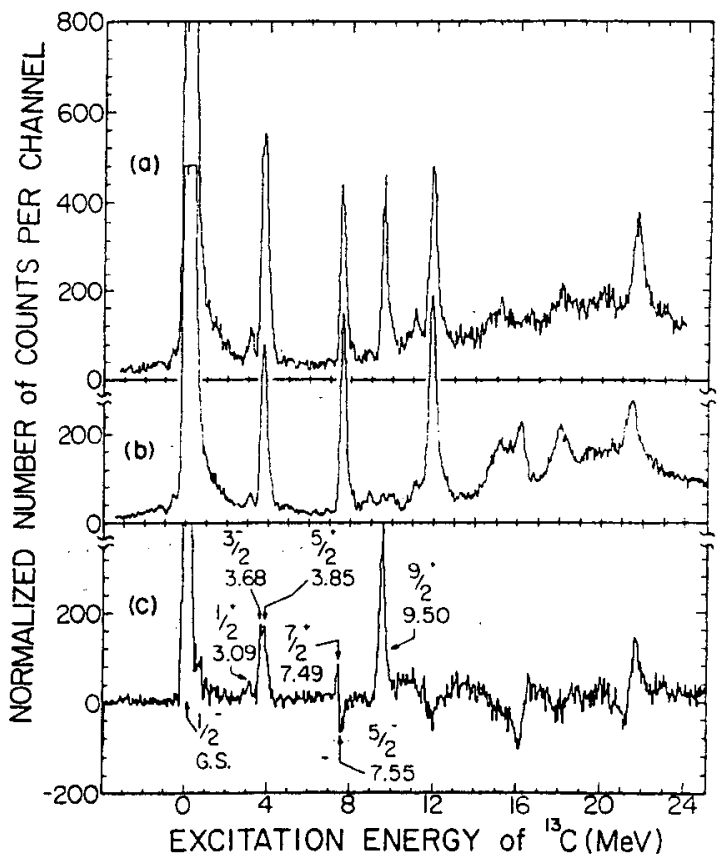

FIG. 1. Summed normalized yield spectra (a) $Y_{\pi}$ and (b) $Y_{\pi}+$ for ${ }^{13} \mathrm{C}\left(\pi^{ \pm}, \pi^{ \pm \prime}\right)$ at $T_{\pi}=162 \mathrm{MeV}\left(\theta_{1 \mathrm{ab}}=62^{\circ}\right.$, $68^{\circ}, 74^{\circ}, 80^{\circ}, 86^{\circ}$ ), and (c) difference between $Y_{\pi} \cdot$ and $Y_{\pi}{ }^{+}$. 
shifted towards lower excitation energy in the $\pi^{+}$ spectra in comparison with the $\pi^{-}$spectra as is apparent in the difference spectrum [Fig. 1(c)].

We prefer to represent the differences between the cross sections $\sigma_{\pi}$ - and $\sigma_{\pi}+$ by the parameter $A=\left(\sigma_{\pi}--\sigma_{\pi+}\right) /\left(\sigma_{\pi}-+\sigma_{\pi+}\right)$ rather than by the ratio $R=\sigma_{\pi}-/ \sigma_{\pi}+$. Values of $A$ obtained at the maxima of the differential cross sections are presented in Fig. 2. For the $9.5-\mathrm{MeV}$ state $A=+0.8 \pm 0.2$, consistent with a pure neutron excitation. For a state or a group of states at $E_{x} \approx 16 \mathrm{MeV}, A$ $=-0.6 \pm 0.2$ indicating an almost pure proton excitation. For other states the values of $A$ range from about -0.4 to +0.4 , more in line with previously observed enhancement factors. [The ratio $R=1.86 \pm 0.16$ for ${ }^{18} \mathrm{O}\left(2^{+}\right.$) (Ref. 2) corresponds to a value of $A=+0.30 \pm 0.04$.]

Of the "single-neutron states" of ${ }^{13} \mathrm{C}$ known" from ${ }^{12} \mathrm{C}(d, p)$ we resolve the weakly excited $\frac{1}{2}^{+}$ state at $3.09 \mathrm{MeV}$ and obtain $A=0.3 \pm 0.1$. The $\frac{5}{2}^{+}$ state at $3.85 \mathrm{MeV}$ was not fully resolved from the $\frac{3}{2}^{-}$state at $3.68 \mathrm{MeV}$ but we estimate cross sections from peak fitting and derive $A=0.4 \pm 0.1$. These values of $A$ are only about one-half of the free-neutron value indicating sizable contributions from the protons or from more complex reaction mechanisms.

At forward angles $\left(\theta_{\mathrm{c} . \mathrm{m} .} \leqslant 50^{\circ}\right)$ the unresolved triplet at $7.5 \mathrm{MeV}$ is expected to be dominated by the transition to the collective $\frac{5}{2}^{-}$state $(7.55 \mathrm{MeV})$. This state and the $\frac{3}{2}^{-}$state at $3.68 \mathrm{MeV}$ are often interpreted as a weak-coupling doublet obtained by coupling a $p_{1 / 2}$ neutron to the first excited $2^{+}$

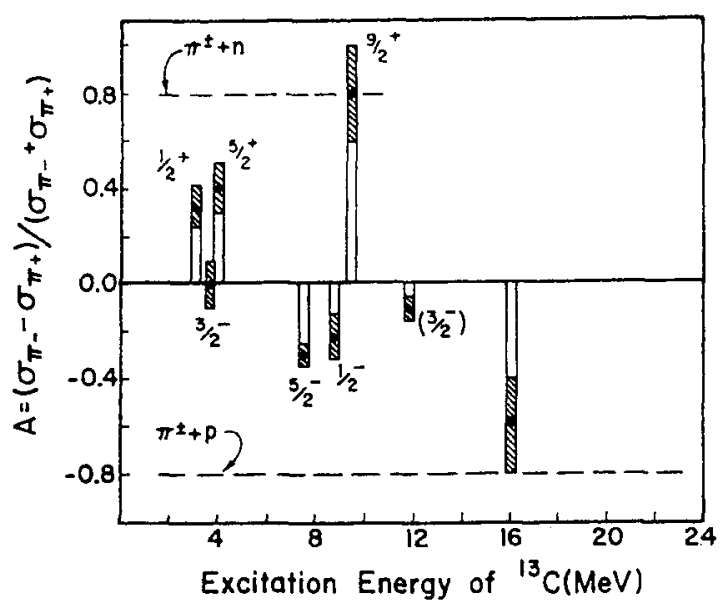

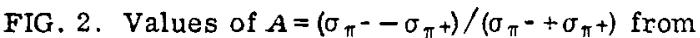
${ }^{13} \mathrm{C}\left(\pi^{ \pm}, \pi^{ \pm \prime}\right)$ at $162 \mathrm{MeV}$ at the maxima of the differential cross sections. Broken horizontal lines: values of $\boldsymbol{A}$ for $\pi^{ \pm}+n$ and $\pi^{ \pm}+p$. state of ${ }^{12} \mathrm{C}$. At forward angles the $3.68-\mathrm{MeV}$ state is almost equally excited by $\pi^{+}$and $\pi^{-}(A$ $\simeq 0$ ) but the $7.5-\mathrm{MeV}$ triplet is a factor of 2 more strongly excited by $\pi^{+}$than by $\pi^{-}(A=-0.3 \pm 0.1)$. [At larger angles $\left(\theta_{\text {c.m. }} \approx 50^{\circ}\right)$ the $7.5-\mathrm{MeV}$ triplet is nearly equally excited by $\pi^{+}$and $\pi^{-}$. $]$The different values of $A$ for the weak-coupling states imply a state dependence of the blocking of the quadrupole strength of ${ }^{12} \mathrm{C}$ by the extra neutron. This can be understood in terms of the major components of the wave functions in simple $j-j$ coupling. If the excited core $\left(2^{+}, T=0\right)$ consisted of only a $\left(p_{3 / 2}\right)^{-1}\left(p_{1 / 2}\right)^{+1}$ one-particle, one-hole $(1 \mathrm{p}-1 \mathrm{~h})$ structure, the addition of a $p_{1 / 2}$ neutron would generate $2 \mathrm{p}-1 \mathrm{~h}$ states with $J^{\pi}=\frac{1}{2}^{-}, \frac{5}{2}_{1}{ }^{-}, \frac{3}{2}_{2}^{-}$, and $\frac{5}{2}^{-}$. The isospin structure of the $\frac{1}{2}^{-}, \frac{3}{2}_{2}^{-}$, and $\frac{5}{2}^{-}$states would be such that only protons could be involved in the transition. Apparently the "collective" $\frac{5}{2}^{-}$state at $7.55 \mathrm{MeV}$ has a large component of $2 p-1$ h structure leading to the observed $\pi^{+}$enhancement. Clearly the excited core is not a pure $\left[\left(p_{3 / 2}\right)^{-1}\left(p_{1 / 2}\right)^{+1}\right]_{2}+$ state $(1 p-1 \mathrm{~h})$ and the experimental value of $A$ for the $\frac{5}{2}^{-}$state $(A=-0.3 \pm 0.1)$ is smaller than the free-proton value. Thus neutrons must also be involved in the transition. The $\frac{3}{2}_{1}$ - state can be reached by both proton and neutron excitations even if the core were a pure $1 \mathrm{p}-1 \mathrm{~h}$ state. Still, the experimental value of $A$ $\simeq 0$ requires additional neutron contributions since the simple $1 \mathrm{p}-0 \mathrm{~h}$ to $2 \mathrm{p}-1 \mathrm{~h}$ transitions would favor proton excitations.

The spin and parity assignment, $J^{\pi}=\frac{3}{2}^{-}$, for the $9.5-\mathrm{MeV}$ state is quite uncertain. ${ }^{5 \cdot 10-14}$ In low-energy electron scattering no $M 1$ strength could be detected. ${ }^{10}$ Two- and three-particletransfer reactions ${ }^{11,12}$ quite strongly suggest a high-spin state at this energy and recent highenergy electron-scattering data ${ }^{13}$ yield $J^{\pi}=\frac{9}{2}^{+}$. A $\frac{7}{2}^{+}$and ${ }^{2} \frac{9}{2}^{+}$state have been predicted around this energy by Lane ${ }^{15}$ and later by several other authors (e.g., Baker ${ }^{16}$ and Jäger et $a l .^{17}$ ) as members of a weak-coupling multiplet based on a $d_{5 / 2}$ neutron coupled to the first excited $2^{+}$state in ${ }^{12} \mathrm{C}$. Such a structure is consistent with the extremely small neutron width from ${ }^{12} \mathrm{C}+n$ elastic scattering ${ }^{14}$ and the preferential decay of the $9.5-\mathrm{MeV}$ state in ${ }^{13} \mathrm{C}$ or its mirror at $9.0 \mathrm{MeV}$ in ${ }^{13} \mathrm{~N}$ by neutron or proton emission, respectively, to the $2^{+}$state in ${ }^{12} \mathrm{C}$ (see references in Ref. 12). The wave function of the $\frac{2}{2}^{+}$member of the weakcoupling multiplet has a very large overlap with the wave function of $\mathrm{a}_{2}{ }^{+}$state at about $9.5 \mathrm{MeV}$ predicted by the intermediate-coupling model (Ref. 18). $\mathrm{A}_{\frac{2}{2}}{ }^{+}$state of similar structure was 
also found close to $9.5 \mathrm{MeV}$ in a shell-model calculation. ${ }^{19}$

We remark that an inelastic transition to the $\left[\left(2^{+}, T_{0}=0\right) \otimes\left(d_{5 / 2}\right)\right]_{1 / 2}+\ldots, 9 / 2+, T=1 / 2$ weak-coupling states should involve mainly neutrons. The ground state (g.s.) of ${ }^{13} \mathrm{C}$ may be represented by a mixture of $p$-shell $(m+1)$-particle, $m$-hole states $(m=0,1,2)$. If we assume the excited ${ }^{12} \mathrm{C}\left(2^{+}\right)$to be a mixture of $p$-shell $n$-particle, $n$ hole states $(n=1,2,3,4)$ with $T_{0}=0$, the weakly coupled $d_{5 / 2}$ nucleon can only be a neutron. This means that only the promotion of a $p$-shell neutron to the $d_{5 / 2}$ shell can contribute to the transi-tion if the g.s. and the multiplet states are connected by a single-particle operator. Thus the strong $\pi^{-}$enhancement for the $9.5-\mathrm{MeV}$ state and the $\frac{9}{2}+$ assignment from electron scattering ${ }^{13}$ suggest that this state is the the $\frac{9}{2}^{+}$member of the multiplet. The $\frac{7}{2}^{+}$member ${ }^{20}$ is probably the 7.59 $\mathrm{MeV}$ state of the unresolved triplet at $7.5 \mathrm{MeV}$. The $\pi^{+}$enhancement of the $7.5-\mathrm{MeV}$ triplet is observed only at forward angles $\left(s 50^{\circ}\right)$. At larger angles $\left(\succsim 50^{\circ}\right)$ the $\sigma_{\pi}+(\theta)$ and $\sigma_{\pi}-(\theta)$ are quite similar. This may be due to relatively large contributions from the $\frac{7}{2}^{+}$state which should be enhanced in $\pi^{-}$scattering like the $9.5-\mathrm{MeV}$ state.

Since we assume the $2^{+} \otimes d_{5 / 2}$ configuration to be excited by a one-step process (primarily by the promotion of one of several $p_{3 / 2}$ neutrons to the $d_{5 / 2}$ shell), the transition can proceed by an orbital angular momentum transfer $\Delta L$ of at most 3. Thus the minimum total angular momentum transferred to the target, $\Delta J=4$, for the $\frac{1}{2}^{-} \rightarrow^{\frac{9}{2}}+$ transition, is obtained by a spin flip of the neutron. The shape of the angular distribution for the $\frac{9}{2}^{+}$state has the appearance of a $\Delta L=5$ transition which we obtained from a spin-nonflip distorted-wave impulse-approximation (DWIA) calculation using the code ${ }^{21}$ DWPI with a complex collective form factor. However, angular distributions for spin-flip transitions are known ${ }^{22}$ to peak at larger angles than spin-nonflip transitions of the same $\Delta L$. In fact, our qualitative arguments are being confirmed by recent microscopic DWIA calculations of Lee and Kurath ${ }^{23}$ which use intermediate-coupling model wave functions ${ }^{18}$ for the g.s. and the lowest $\frac{9}{2}^{+}$state. These calculations give a good fit to our data for'the state at 9.5 $\mathrm{MeV}$ with $\Delta L=3$ and $\Delta S=1$.

States of a $2^{+} \otimes d_{5 / 2}$ configuration can also be obtained by coupling a $d_{5 / 2}$ particle to a $2^{+}, T_{0}=1$ state of the core resulting in isospins $T=\frac{1}{2}$ or $T$ $=\frac{3}{2}$. The isospin coupling coefficients for the $T$ $=\frac{1}{2}$ states are such that the protons should be in- volved in the transition more strongly than the neutrons and for the $T=\frac{3}{2}$ states neutrons and protons would be expected to contribute more equally. We suggest that the $\pi^{+}$enhanced state (or group of states) at $16 \mathrm{MeV}$ contains components of the $T=\frac{1}{2}$ multiplet states.

In summary, the inelastic scattering of $\pi^{+}$and $\pi^{-}$on ${ }^{13} \mathrm{C}$ yielded a number of striking results. Two transitions were found to be consistent with pure neutron and pure proton excitations. Qualitative arguments were presented to explain these data as transitions to states of principally $2^{+} \otimes d_{5 / 2}$ weak-coupling structure. The weak transitions to the "single-neutron states" at 3.09 and 3.85 $\mathrm{MeV}$ were only moderately enhanced in $\pi^{-}$scattering suggesting significant proton particle-hole components in the wave functions or sizable contributions from two-step processes. The excitation of the $\left[2^{+} \otimes p_{1 / 2}\right]_{3 / 2}-, 5 / 2-$ weak-coupling states showed a state dependence of the blocking of the quadrupole strength of the ${ }^{12} \mathrm{C}$ core. Finally, a difference in the structure of $\pi^{-}$and $\pi^{+}$spectra at about $21 \mathrm{MeV}$ was observed, similar to results ${ }^{8}$ for ${ }^{12} \mathrm{C}+\pi^{*}$, indicating isospin mixing between $T=\frac{1}{2}$ and $T=\frac{3}{2}$ states. The experimental angular distributions and calculations will be presented in a forthcoming publication.

The authors are indebted to Dr. D. Kurath, Dr. T.-S. H. Lee, Dr. B. F. Bayman, and Dr. P. J. Ellis for helpful discussions. The authors also wish to thank Dr. Lee and Dr. Kurath for permission to quote some of their results prior to publication, and Dr. R. J. Macek for providing the ${ }^{13} \mathrm{C}$ target.

This work was supported in part by the U. S. Department of Energy and the Swiss Institute of Nuclear Physics.

${ }^{1}$ J. P. Egger et al., Phys. Rev. Lett. 39, 1608 (1977).

${ }^{2}$ S. Iversen et al., Phys. Rev. Lett. 40, 17 (1978), and Phys. Lett. 82B, 51 (1979).

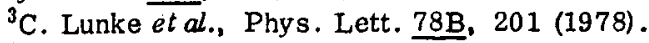

${ }^{4} \mathrm{C}$. L. Morris et al., in Proceedings of the Second International Conference on Meson-Nuclear Physics, Houston, Texas, 5-9 March 1979 (unpublished).

${ }^{5} \mathrm{~F}$. Ajzenberg-Selove, Nucl. Phys. A268, 1 (1976)

${ }^{6} \mathrm{H}$. A. Thiessen and S. Sobottka, Los Alamos Scientific Laboratory Report No. LA-4534-MS (unpublished), and references therein.

${ }^{7}$ Kindly provlded by Dr. R. Macek of group MP-13, Los Alamos Scientific Laboratory.

${ }^{8} \mathrm{C}$. L. Morris et al., to be published. 
${ }^{9}$ S. E. Darden et al., Nucl. Phys. A208, 77 (1973).

${ }^{10} \mathrm{G}$. Wittwer et al., Phys. Lett. 30B, 634 (1969).

${ }^{11}$ N. Anyas-Weis et al., Phys. Rev. 12C, 201 (1974).

${ }^{12}$ C. H. Holbrow et al., Phys. Rev. C 9, 902 (1974).

${ }^{13} \mathrm{D}$. I. Sober, private communication.

${ }^{14}$ W. Galati et al., Phys. Rev. C 5, 1508 (1972).

18 A. M. Lane, Rev. Mod. Phys. 32, 519 (1960).

${ }^{16}$ F. C. Barker, Nucl. Phys. 28,96 (1971).

${ }^{17}$ H. U. Jäger et al., Nucl. Phys. A171, 16 (1971).

${ }^{18}$ S. Cohen and D. Kurath, Nucl. Phys. 73 , 1 (1965);
D. Kurath, private communication.

${ }^{19}$ D. J. Millener and D. Kurath, Nucl. Phys. A255, $31 \overline{5}$ (1975).

${ }^{20}$ The locations of the $\frac{1}{2}^{+}, \frac{3}{2}^{+}$, and $\frac{5}{2}+^{+}$members of the multiplet are uncertain.

${ }^{21}$ R. A. Eisenstein and G. A. Miller, Comp. Phys. Commun. 11, 95 (1976).

${ }^{22}$ G. E. Walker and E. Siciliano, private communication; C. Olmer etal., Phys. Rev. Lett. 43, 612 (1979).

${ }^{23}$ T. S. H. Lee and D. Kurath, private communication.

\title{
Static Polarization Effects in the Nucleus-Nucleus Potential
}

\author{
Fl. Stancu \\ Institut de Physique, Sart Tilman, B-4000 Liège 1, Belgium
}

(Received 25 July 1979)

\begin{abstract}
This letter presents a study of a simple way to relax the frozen-configuration assumption in the calculation of the real part of the nucleus-nucleus potential. The proposed model allows the thickness of the surface layer of each nucleus to vary from the outer side to the interacting side giving rise to polarized shapes which simulate a neck formation. The result is compared to other potentials available in the literature.
\end{abstract}

The calculation of the nucleus-nucleus potential starting from an energy-density formalism is very convenient when simplifying assumptions about the density of the composite system are made. A common starting point is the frozenconfiguration assumption or the sudden approximation ${ }^{1-5}$ according to which the density of the whole system is taken as the sum of the asymptotic individual densities at any separation distance between the interacting nuclei. In practice such a simplification allows one to calculate the interaction potential between any pair of nuclei once the densities are known.

In this Letter I propose a way to relax the frozen-configuration assumption by trying to maintain the simplicity of the calculations. Under mutual influence the nuclei deviate from their spherical shape and I try to describe this polarization effect by a gradual change of the surface layer thickness from an equilibrium value $a_{0}$ at the outer side to a value $a$ at the interacting side of each nucleus. This value is found by minimizing the potential with respect to $a$ at each separation distance $s_{0}$ between the surfaces, with

$$
\mathrm{s}_{0}=R-R_{1}-R_{2} \text {, }
$$

where $R$ is the distance between the centers of the nuclei, and $R_{1}$ and $R_{2}$ are the nuclear.radii.
In this way I extend the formalism of Ref. 4 according to which one defines the nucleus-nucleus potential $\tilde{V}\left(s_{0}, a\right)$ as the difference between the binding energy of the composite system at $s_{0}$ and the binding energy of the nuclei separated at infinity. The Skyrme-interaction density functional ${ }^{6}$ is used to calculate the binding energies. For the composite system the density is taken as the sum of the individual densities and the kinetic energy densities of the composite system and of the individual nuclei are described by the ThomasFermi approximation. The advantage of these approximations is that the Skyrme-interaction energy density $H$ reduces to an algebraic function of the densities $\rho_{1}$ and $\rho_{2}$ only. Then the potential can be written as the volume integral

$$
\begin{aligned}
\tilde{V}\left(s_{0}, a\right)=\int\left[H\left(\rho_{1}{ }^{a}+\rho_{2}{ }^{a}\right)-\right. & H\left(\rho_{1}{ }^{a} 0\right) \\
& \left.-H\left(\rho_{2}{ }^{a} 0\right)\right] d v .
\end{aligned}
$$

The parameter $a_{0}$ is the equilibrium value of the surface layer thickness for an individual nucleus. As Skyrme-interaction parameters I have chosen the set $^{6}$ SII which gives for a semi-infinite slab $a_{0}=0.48 \mathrm{fm}$ and $E_{\text {s urf }}=22.38 \mathrm{MeV} / \mathrm{fm}^{2}$ when the Thomas-Fermi approximation is used for the kinetic energy density as defined in Ref. 6. At infinity the densities $\rho_{1}$ and $\rho_{2}$ are described by 


\title{
Isospin Mixing of $4^{-}$Particle-Hole States in ${ }^{16} \mathrm{O}$
}

D. B. Holtkamp, W. J. Braithwaite, W. Cottingame, S. J. Greene, R. J. Joseph, and C. Fred Moore University of Texas, Austin, Texas 78712

and

C. L. Morris, J. Piffaretti, E. R. Siciliano, and H. A. Thiessen

Los Alamos Scientific Laboratory, Los Alamos, New Mexico 87545

and

D. Dehnhard

University of Minnesota, Minneapolis, Minnesota 55455

(Received 31 March 1980)

\begin{abstract}
Transitions to $\left(4^{-}, T=0\right)$ states in ${ }^{16} \mathrm{O}$ at 17.79 and $19.80 \mathrm{MeV}$ are found to be strongly asymmetric in $\pi^{+}$vs $\pi^{-}$inelastic scattering; in contrast, the $\pi^{+}$and $\pi^{-}$cross sections for the $\left(4^{-}, T=1\right)$ state at $18.99 \mathrm{MeV}$ are equal. Three-state isospin mixing is proposed as an explanation. Off-diagonal charge-dependent mixing matrix elements of $-147 \pm 25$ and $-99 \pm 17 \mathrm{keV}$ are obtained.
\end{abstract}

PACS numbers: $21.10 . \mathrm{Hw}, 25.80 .+\mathrm{f}$

We report the first observation of isospin $\mathrm{mix}-$ ing among three nuclear states which was detected by comparing $\pi^{+}$and $\pi^{-}$inelastic scattering. This technique has been used to separate proton and neutron components of nuclear excitations at pion energies near the $(3,3)$ resonance, ${ }^{1}$ where the ratio of the cross sections for $\pi^{+}$vs $\pi^{-}$scat-tering from protons (neutrons) is about 9:1 (1:9). Furthermore, a direct comparison of $\pi^{+}$and $\pi^{-}$ inelastic scattering at these energies from selfconjugate nuclei provides a method of detecting isospin impurities of the excited nuclear states." In previous cases ${ }^{3-7}$ of strong isospin mixing $\left({ }^{8} \mathrm{Be},{ }^{12} \mathrm{C}\right.$, and $\left.{ }^{16} \mathrm{O}\right)$, only two states were believed to be involved.

Our study of $\pi^{+}$and $\pi^{-}$inelastic scattering from ${ }^{16} \mathrm{O}$ at large momentum transfer was carried out on the Energetic Pion Channel and Spectrometer (EPICS) at the Clinton P. Anderson Meson Physics Facility, which has been described in detail elsewhere. ${ }^{8}$ Ice targets of 160 and $320 \mathrm{mg} / \mathrm{cm}^{2}$ thickness were used. The incident pion energy was $164 \mathrm{MeV}$, and the angular range was from $53^{\circ}$ to $89^{\circ}$ (laboratory). Typical energy resolution was $350 \mathrm{keV}$ full width at half maximum (FWHM). The absolute cross sections were determined by scattering $\pi^{+}$and $\pi^{-}$from the hydrogen in the ice targets and using the calculated $\pi-p$ cross sections of Dodder. ${ }^{9}$ The absolute normalization is believed to be accurate to approximately $10 \%$ whereas the relative $\pi^{+} / \pi^{-}$uncertainty is about $5 \%$.

Spectra obtained from $\pi^{+}$and $\pi^{-}$scattering at a laboratory angle of $77^{\circ}$ are shown in Fig. 1 .
The $\left(4^{\circ}, T=0\right)$ states at 17.79 and $19.80 \mathrm{MeV}$ show large $\pi^{+} / \pi^{-}$asymmetries, but the $\left(4^{-}, T=1\right)$ state at $18.98 \mathrm{MeV}$ does not. Yields for these states were obtained by fitting the region between 16 and $23 \mathrm{MeV}$ with a polynomial background and four peaks of fixed line shape, determined by the line shape of the elastic peak for each thickness of target. The relative energies of the three $4^{-}$ states were fixed at values obtained in previous studies, ${ }^{10,11}$ while the state at $\sim 20.5 \mathrm{MeV}$ was fit when possible (its angular distribution appears to have a minimum near the $4^{-}$maximum and

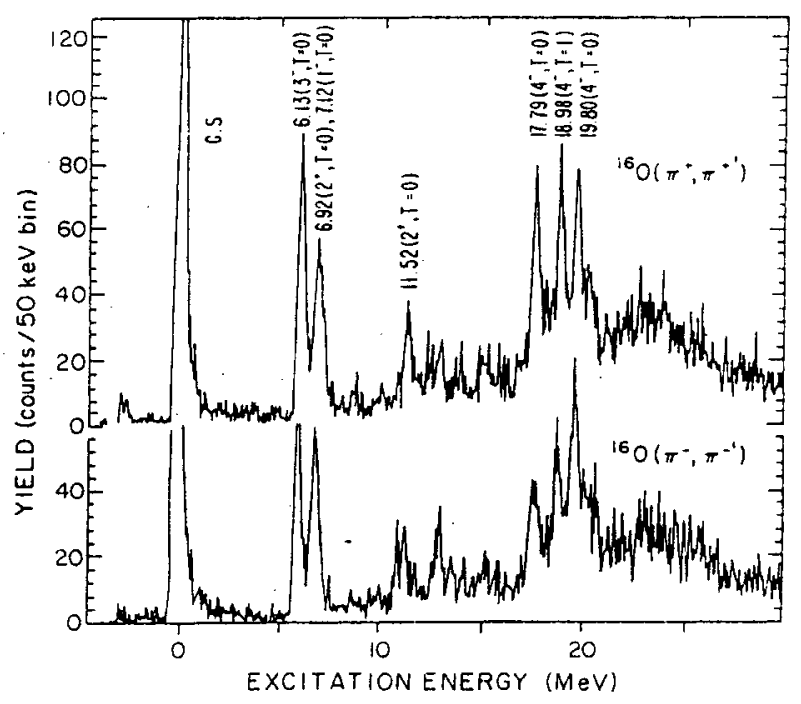

FTG. 1. Energy loss spectra for $\pi^{+}$and $\pi^{-}$at an energy of $164 \mathrm{MeV}$ and a laboratory angle of $77^{\circ}$. 
may be a $3^{-}$state). From the angular distributions shown in Fig. 2, one may obtain the following ratios of the summed yields for $\pi^{+}$vs $\pi^{-}$:

(1) $17.79 \mathrm{MeV}\left(4^{-}, T=0\right), R\left(\pi^{+} / \pi^{-}\right)=1.59 \pm 0.12$;

(2) $18.98 \mathrm{MeV}\left(4^{-}, T=1\right), R\left(\pi^{+} / \pi^{-}\right)=0.964 \pm 0.080$; and (3) $19.80 \mathrm{MeV}\left(4^{-}, T=0\right), R\left(\pi^{+} / \pi^{-}\right)=0.605$ \pm 0.049 . (Errors quoted are statistical.) From charge symmetry, these ratios are expected to be unity. For example, the low-lying $\left(3^{-}, T=0\right)$ state at $6.13 \mathrm{MeV}$ has a $\pi^{+} / \pi^{-}$cross section ratio of $0.98 \pm 0.04$ at $45^{\circ}$, which is near its maximum.

These states in ${ }^{16} \mathrm{O}$, which have a $\left(d_{5 / 2}, p_{3 / 2}{ }^{-1}\right)$ configuration, have been seen in one-nucleon transfer reactions ${ }^{10}$ and in inelastic proton scattering at medium energies. ${ }^{11}$ The $18.98-\mathrm{MeV}$ state $\left(4^{-}, T=1\right)$ has also been observed in highenergy electron scattering. ${ }^{12,13}$ The excitation energies of these states are well above particle threshold, and because medium-energy pions are strongly absorbed, one may expect $\pi^{+} / \pi^{-}$ asymmetries to result from differences in proton and neutron single-particle wave functions. These continuum effects in the reaction channels have been investigated by Siciliano and Weiss ${ }^{14}$ and cannot explain the asymmetries observed. The authors of Ref. 14 conclude that continuum effects would result in a systematic $\pi^{-}$bias in the cross sections for both $T=0$ and $T=1$ states, rather

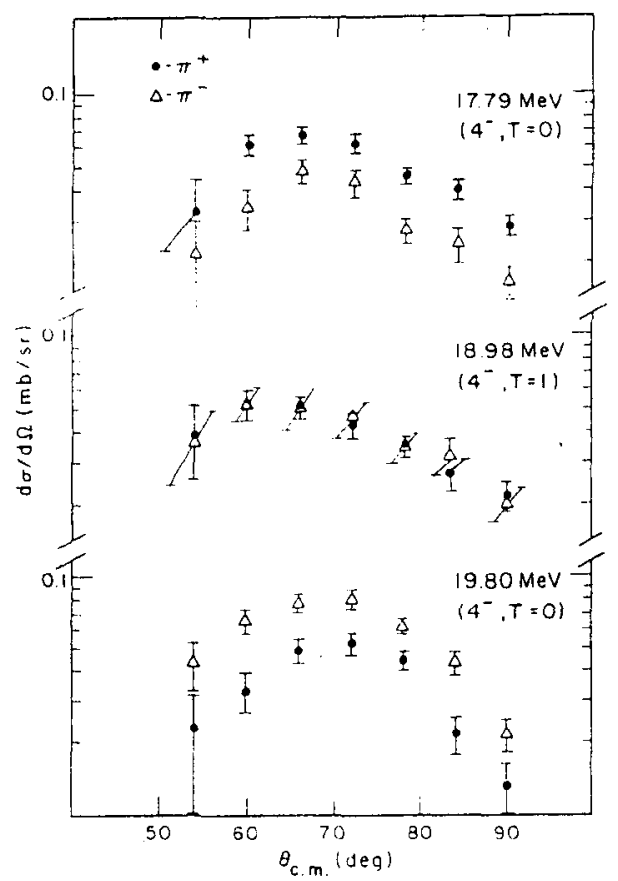

FIG. 2. Center-of-mass differential cross sections for $\pi^{ \pm}$scattering at $164 \mathrm{MeV}$. than a reversal of the $\pi^{+} / \pi^{-}$asymmetry as observed for the $T=0$ states. Our interpretation of the asymmetries for pion excitation of these states is that they are substantially mixed in isospin. In previous cases of two-state isospin mixing, ${ }^{2-7}$ the $T_{<}$state becomes mostly a proton state ( $\pi^{+}$enhanced) and the $T>$ state is predominantly a neutron state $\left(\pi^{-}\right.$enhanced). For ${ }^{16} \mathrm{O}$, three $4^{-}$ states are known within $2 \mathrm{MeV}$ so that three-state mixing has to be considered. The qualitative picture of the mixing in ${ }^{16} \mathrm{O}$ is that the two $T=0$ states mix through the $T=1$ state with nearly equal magnitudes. The lower state mixes with the middle state, resulting in the lower level becoming mostly a proton state and the middle level becoming mostly a neutron state. However; when the middle state mixes with the upper state, the energy denominator of the mixing changes sign, which results in the state above becoming primarily neutron in composition while the middle state gains in proton strength from this second mixing. Thus, the $T=1$ state retains nearly equal proton and neutron amplitudes, whereas the lower level becomes mostly a proton state and the upper level mostly a neutron state.

If we assume that the charge-dependent part of the nuclear Hamiltonian, $H_{c d}$, may be treated as a small perturbation, we may write the physical states (labeled $\left|A^{\prime}\right\rangle,\left|B^{\prime}\right\rangle$, and $\left\langle C^{\prime}\right\rangle$ ) as linear combinations of the unperturbed states of pure isospin (labeled $|A\rangle,|B\rangle$, and $|C\rangle$ ) as

$$
\begin{aligned}
& \left|A^{\prime}\right\rangle=|A\rangle+\epsilon_{1}|B\rangle+\epsilon_{3}|C\rangle, \\
& \left|B^{\prime}\right\rangle=-\epsilon_{1}|A\rangle+|B\rangle+\epsilon_{2}|C\rangle, \\
& \left|C^{\prime}\right\rangle=-\epsilon_{3}|A\rangle-\epsilon_{2}|B\rangle+|C\rangle,
\end{aligned}
$$

where $\epsilon_{1}=\left\langle A\left|H_{C d}\right| B\right\rangle /\left(E_{A}-E_{B}\right), \epsilon_{2}=\left\langle B\left|H_{C d}\right| C\right\rangle /$ $\left(E_{B}-E_{C}\right)$, and $\epsilon_{3}=\left\langle A\left|H_{c d}\right| C\right\rangle /\left(E_{A}-E_{C}\right)$. In these calculations we will ignore terms of order $\epsilon^{2}$. The unperturbed $4^{-}$states of ${ }^{16} \mathrm{O}$ must be configuration mixed as well because the single-particle, single-hole (1p-1h) configurations can result in only two states $(T=0,1)$. Therefore, the unperturbed states are written as

$$
\begin{aligned}
& |A\rangle=\alpha|1 \mathrm{p}-1 \mathrm{~h}, T=0\rangle+\sum_{i} \alpha_{i}\left|\psi_{i}, T=0\right\rangle, \\
& |B\rangle=\beta|1 \mathrm{p}-2 \mathrm{~h}, T=1\rangle+\sum_{i} \beta_{i}\left|\psi_{i}, T=1\right\rangle, \\
& |C\rangle=\gamma|1 \mathrm{p}-1 \mathrm{~h}, T=0\rangle+\sum_{i} \gamma_{i}\left|\psi_{i}, T=0\right\rangle,
\end{aligned}
$$

where $\psi_{i}$ labels possible multiparticle-multihole configurations.

At this point we make the assumption that the distorted-wave impulse approximation (DWLA) provides an adequate description of the pion-nu- 
cleus interaction. ${ }^{15}$ This assumption treats the transition operator as a one-body operator on the nuclear space. We note that even from the complicated ground state of ${ }^{10} \mathrm{O}$, which contains substantial $2 \mathrm{p}-2 \mathrm{~h}$ admixtures, ${ }^{16}$ the one-body density matrix elements for $2 p-2 h$ to $1 p-1 h$ transitions to the "stretched" 4 " states are identically zero, for $2 \mathrm{p}-2 \mathrm{~h}$ configurations from the $\rho_{1 / 2}$ shell. Also, contributions of $2 p-2 h$ to $3 p-3 h$ transitions are dramatically reduced relative to the core to $1 p-$ 1h terms. Thus, within the model, only the 1p1h amplitudes of Eq. (2) are reached.

If we further assume that the energy dependence of the $\pi$-nucleon interaction is dominated by the $(3,3)$ channel, the DWIA $\pi$-nucleus amplitude for a $1 \mathrm{p}-1 \mathrm{~h}$, unnatural-parity transition from an even-even nucleus may be written as ${ }^{14}$

$$
\Lambda_{\varphi_{z}}{ }^{T}=F\left[\left(2+\varphi_{z}\right)+(-1)^{T}\left(2-\varphi_{z}\right)\right],
$$

where $\varphi_{z}= \pm 1\left(\pi^{ \pm}\right)$and $T(=0,1)$ is the nuclear isospin. The proton and neutron single-particle wave functions are assumed to be identical, and the differences between $\pi^{+}$and $\pi^{-}$distorted waves are neglected. The factor $F$ contains all of the details common to the $\pi^{ \pm}$excitation of the unper turbed states at the same incident pion energy and momentum transfer: distorted waves, radial integrals, angular-momentum recoupling coefficients, etc. Then, to order $\epsilon_{i}$, the cross sections for $\pi^{ \pm}$scattering to the isospin mixed states may be written as

$$
\begin{aligned}
& \sigma_{\varphi_{z}{ }^{A^{\prime}}}=|\hat{F}|^{2} 16 \hat{\alpha}\left[\hat{\alpha}+2 \hat{\gamma} \epsilon_{3}+\varphi_{z} \epsilon_{1}\right], \\
& \sigma_{\varphi_{z}{ }^{\prime}}=|\hat{F}|^{2} 4\left[1+4 \varphi_{z}\left(\hat{\gamma} \epsilon_{2}-\hat{\alpha} \epsilon_{1}\right)\right], \\
& \sigma_{\varphi_{z}{ }^{C^{\prime}}}=|\hat{F}|^{2} 16 \hat{\gamma}\left[\hat{\gamma}-2 \hat{\alpha} \epsilon_{3}-\varphi_{z} \epsilon_{2}\right],
\end{aligned}
$$

where $|\hat{F}|^{2}(\hat{\alpha}, \hat{\gamma})$ is $|F|^{2}(\alpha, \gamma)$ multiplied (divided) by $\beta^{2}(\beta)$ because in the absence of a full DWIA calculation, the absolute magnitude of the $1 p-1 h$ strength cannot be determined.

The six summed cross sections for $\pi^{ \pm}$scattering to the three states were used with the above expressions to solve for the six quantities $\hat{\alpha}, \hat{\gamma}$, $\epsilon_{1}, \epsilon_{2}, \epsilon_{3}$, and $|\hat{F}|^{2}$. The results of the calculation are shown in Table I. An identical analysis which kept all terms to order $\epsilon^{2}$ yielded results unchanged from the present work.

The deduced $1 \mathrm{p}-1 \mathrm{~h}$ amplitudes for these states are in qualitative agreement with the relative spectroscopic factors obtained by Mairle ${ }^{9}$ in an ${ }^{17} \mathrm{O}(d, t){ }^{16} \mathrm{O}$ experiment when the effects of isospin mixing are included, i.e., the 17.79-MeV state has less neutron $p-h$ strength than the $19.80-\mathrm{MeV}$ state, relative to the $18.98-\mathrm{MeV}$ state. The fact that $\hat{\alpha}^{2}+\hat{\gamma}^{2} \leqslant 0.54$ indicates further fragmentation of the $T=0$ strength in the excitation spectrum. Because there is no experimental evidence for these other $T=0$ states, we assume that no more than the two observed $\left(4^{-}, T=0\right)$ levels can mix significantly with the $\left(4^{-}, T=1\right)$ state. If one assumes that $\beta$ is unity, as is suggested by the studies of Mairle, ${ }^{10}$ and neglects isospin mixing between the $1 p-1 h$ and $3 p-3 h$ configurations, the $1 \mathrm{p}-1 \mathrm{~h}\left\langle T=0\left|H_{c d}\right| T=1\right\rangle$ matrix element obtained is about $-240 \pm 40 \mathrm{keV}$. This value is somewhat uncertain, however, because small $3 \mathrm{p}-3 \mathrm{~h}$ amplitudes in the $T=1$ state may significantly affect the exact value of the $1 \mathrm{p}-1 \mathrm{~h}$ matrix element obtained, as well as the effects of isospin mixing between the $1 p-1 h$ and $3 p-3 h$ configurations. To determine $1 p-$ $1 \mathrm{~h}$ or $3 p-3 \mathrm{~h}$ matrix elements accurately, it may be necessary to interpret our results within the

TABLE I. Values for the physical and unperturbed energies, configuration-mixing parameters, and the charge-dependent mixing matrix element are shown. Errors on $\hat{\alpha}, \hat{\gamma}_{,}\left\langle A\left|H_{c d}\right| B\right\rangle,\left\langle B\left|H_{c d}\right| C\right\rangle,\left\langle A\left|H_{c d}\right| C\right\rangle$, and $|\hat{F}|^{2}$ are estimates based on the experimental errors. The DWIA parameter $|\hat{F}|^{2}=0.0625 \pm 0.0034 \mathrm{mb} / \mathrm{sr}$. The values for the unperturbed energies do not include a diagonal charge-dependent energy shift.

\begin{tabular}{ccc}
\hline $\begin{array}{c}\text { Physical energy } \\
(\mathrm{MeV})\end{array}$ & $\begin{array}{c}\text { Unperturbed energy } \\
(\mathrm{MeV})\end{array}$ & $1 \mathrm{p}$-1h amplitudes \\
\hline $17.79 \pm 0.02$ & $17.81 \pm 0.02$ & $\hat{\mathrm{u}}=0.510 \pm 0.043$ \\
$18.98 \pm 0.02$ & $18.98 \pm 0.02$ & \\
$1.8 .80 \pm 0.02$ & $19.78 \pm 0.02$ & $\hat{\gamma}=0.529 \pm 0.046$ \\
& Charge-dependent mixing matrix elements \\
& $\left\langle A\left|H_{c d}\right| B\right\rangle=-147 \pm 25 \mathrm{keV}$ & \\
& $\left\langle B\left|H_{c d}\right| C\right\rangle=-99 \pm 17 \mathrm{keV}$ & \\
& $\left.A\left|H_{c d}\right| C\right\rangle=17 \pm 30 \mathrm{keV}$ \\
\hline
\end{tabular}


framework of large-basis shell-model wave functions. Such an analysis is in progress. ${ }^{17}$

In conclusion, large asymmetries in $\pi^{+}$vs $\pi^{-}$ excitations of $4^{-}$states in ${ }^{16} \mathrm{O}$ are interpreted as evidence of substantial isospin mixing among the states. Off-diagonal charge-dependent matrix elements of $-147 \pm 25$ and $-99 \pm 17 \mathrm{keV}$ are obtained when the data are analyzed with a simple model for three-state mixing. Before definite conclusions can be reached concerning the magnitude of charge-dependent forces in nuclei more experimental and theoretical study is needed; however, intermediate-energy pions will be a useful tool in the study of this aspect of nuclear structure.

This work was supported in part by the U. S. Department of Energy, by the Robert A. Welch Foundation, and by the Swiss Institute for Nuclear Research.

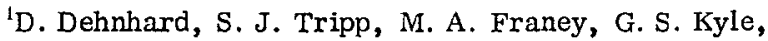
C. L. Morris, R. L. Boudrie, J. Piffaretti, and H. A. Thiessen, Phys. Rev. Lett. $\underline{43}, 1091$ (1979).

${ }^{2}$ C. L. Morris, J. Piffaretti, H. A. Thiessen, W. B. Cottingame, W. J. Braithwaite, R. J. Joseph, I. B. Moore, D. B. Holtkamp, C. J. Harvey, S. J. Greene, C. F. Moore, R. L. Boudrie, and R. J. Peterson, Phys. Lett. 86B, 31 (1979).

${ }^{3}$ J. B. Marion, P. H. Nettles, C. L. Cocke, and G. J. Stephenson, Jr., Phys. Rev. 157, 847 (1967).

${ }^{4}$ E. G. Adelberger, R. E. Marrs, K. A. Snover, and
J. E. Bussoletti, Phys. Rev. C 15, 484 (1977).

${ }^{5}$ W. J. Braithwaite, J. E. Bussoletti, F. E. Cecil, and G. T. Garvey, Phys. Rev. Lett. 29, 376 (1972).

${ }^{6} \mathrm{~J}$. M. Lind, G. T. Garvey, and R. E. Tribble, Nucl. Phys. A276, 25 (1977).

${ }^{7}$ G. J. Wagner, K. T. Knöpfle, G. Mairle, P. Doll, H. Hafner, and J. L. C. Ford, Jr., Phys. Rev. C 16, 1271 (1977).

${ }^{8}$ D. B. Holtkamp, Ph.D. thesis, University of Texas, Austin, Texas, 1979 (unpublished), and Los Alamos Scientific Laboratory Report No. LA-8231-T, 1980 (unpublished).

${ }^{9}$ D. Dodder, private communication.

${ }^{10}$ G. Mairle, G. J. Wagner, P. Doll, K. T. Knöpfle, and H. Breuer, Nucl. Phys. A299, 39 (1978).

${ }^{11}$ R. S. Henderson, B. M. Spicer, I. D. Svalbe, V. C. Officer, G. G. Shute, D. W. Devins, D. L. Friesel, W. P. Jones, and A. C. Attard, Aust. J. Phys. 32 , 411 (1979).

${ }^{12}$ I. Sick, E. B. Hughes, T. W. Donnelly, J. D. Walecke, and G. E. Walker, Phys. Rev. Lett. 23, 1117 (1969).

${ }^{13}$ R. A. Lindgren, R. Hicks, and G. A. Peterson, private communication.

${ }^{14} \mathrm{E}$. R. Siciliano and D. L. Weiss, in Proceedings of the Clinton P. Anderson Meson Physics Facility Workshop on Nuclear Structure with Intermediate Energy Probes, Los Alamos, New Mexico, 14-16 January 1980, Los Alamos Scientific Laboratory Report No. LA-8303-C, 1980 (unpublished), p. 298; and to be published.

${ }^{15}$ See, for example, H. S.-T. Lee and D. Kurath, Phys. Rev. C 21, 293 (1980).

${ }^{16}$ G. E. Walker, Phys. Rev. 174, 1290 (1968), and

Phys. Rev. C $\underline{5}, 1540$ (1972).

${ }^{17} \mathrm{~J}$. Dubach and W. Haxton, private communication. 
(1973).

${ }^{8}$ R. Rosenfelder, Nucl. Phys. A298, 397 (1978);

P. Christillin et al., Phys. Lett. 95B, 344 (1980).

${ }^{9}$ G. Bertsch and S. Tsai, Phys. Rep. 18C, 126 (1975);

S. Shlomo and G. Bertsch, Nucl. Phys. A243, 507 (1975).

${ }^{10}$ N. Auerbach and Nguyen Van Giai, Phys. Lett. 72B, 289 (1978).

${ }^{11} \mathrm{C}$. A. Engelbrecht and R. H. Lemmer, Phys. Rev. Lett. 24, 607 (1970).

${ }^{12}$ A. M. Lane and J. Martorell, Ann. Phys. (N.Y.) 129, $273(1980)$.

${ }^{13}$ T. H. R. Skyrme, Nucl. Phys. 9, 615 (1959);

D. Vautherin and D. M. Brink, Phys. Rev. C $\underline{5}, 626$ (1972).
${ }^{14} \mathrm{H}$. Krivine, J. Treiner, and O. Bohigas, Nucl. Phys. A336, 155 (1980).

${ }^{15} \mathrm{~K}$. F. Liu and Nguyen Van Giai, Phys. Lett. 65B, 23 (1976).

${ }^{16} \mathrm{M}$. Beiner, H. Flocard, Nguyen Van Giai, and P. Quentin, Nucl. Phys. A238, 29 (1975).

${ }^{17}$ N. Auerbach, V. Bernard, and Nguyen Van Giai, Nucl. Phys. A337, 143 (1980).

${ }^{18}$ A. M. Lane and A. Z. Mekjian, Phys. Rev. C 8,1981 (1973); A. Z. Mekjian and W. M. MacDonald, Phys. Rev. C 15, 531 (1977).

${ }^{19} \mathrm{O}$. Bohigas, Nguyen Van Giai, and D. Vautherin, in Proceedings of the International Conference on Nuclear Physics, Berkeley, California, 24-30 August 1980 (to be published), p. 225 .

\title{
Identification of $\Delta S=1$ Transitions in ${ }^{13} \mathrm{C}$ by Measurement of Pion Inelastic Excitation Functions
}

\author{
S. J. Seestrom-Morris, (a) D. Dehnhard, and D. B. Holtkamp \\ University of Minnesota, Minneapolis, Minnesota 55455 \\ and \\ C. L. Morris \\ Los Alamos National Laboratory, Los Alamos, New Mexico 87545
}

(Received 17 February 1981)

\begin{abstract}
Differential cross sections for ${ }^{13} \mathrm{C}\left(\pi, \pi^{\prime}\right)$ were measured between 100 and $300 \mathrm{MeV}$ for momentum transfers of $1.1 \hbar \mathrm{fm}^{-1}$ and $1.4 \hbar \mathrm{fm}^{-1}$. In this energy range the different energy dependences of the spin-dependent and spin-independent parts of the pion-nucleon interaction provide a very sensitive method of discriminating between transitions that proceed with a spin transfer $(\Delta S=1)$ or without a spin transfer $(\Delta S=0)$. Five transitions in ${ }^{13} \mathrm{C}$ were found to be dominated by the $\Delta S=1$ transition density amplitude.
\end{abstract}

PACS numbers: $25.80 .+\mathrm{f}, 27.20 .+\mathrm{n}$

The work of Moore et al. ${ }^{1}$ indicated that new information can be obtained from the measurement of inelastic pion excitation functions. In this Letter we report on the use of excitation functions of inelastic pion scattering to distinguish between $\Delta S=0$ and $\Delta S=1$ transitions, where $\Delta S$ is the spin transfer to the target nucleus. Differential cross sections for two transitions in ${ }^{13} \mathrm{C}$, known to proceed predominantly by $\Delta S=0$, were found to have. energy dependences very different from that of a recently determined ${ }^{2,3}$ pure $\Delta S=1$, pure neutron particle-hole excitation of a stretched state.

Four other transitions were found to be dominated by $\Delta S=1$. Such an effect was also seen in the work of Cottingame et al. ${ }^{4}$ in which natural and unnatural parity transitions in ${ }^{12} \mathrm{C}\left(\pi, \pi^{\prime}\right)$ had dra matically different energy dependences. The explanation $^{5}$ of these different energy dependences is based on two facts. Firstly, at energies near the $[3,3]$ resonance the spin-dependent and spin- independent parts of the pion-nucleus scattering amplitude have quite different energy dependences for a given momentum transfer. Secondly, transitions that involve a spin transfer, $\Delta S=1$, to the target can be caused only by the spin-dependent part of the force and transitions without a spin transfer, $\Delta S=0$, are predominantly due to the spin-independent part of the force.

The pion-micleon scattering amplitude can be written in the following form if the interaction is dominated by the $[3,3]$ resonance, ${ }^{6}$

$$
f\left(k, k^{\prime}\right)=\alpha(k)(2 \cos \theta+i \vec{\sigma} \cdot \hat{n} \sin \theta),
$$

where $k\left(k^{\prime}\right)$ is the pion momentum before (after) the collision, $\theta$ is the scattering angle in the pion-nucleon center-of-mass frame, $\vec{\sigma}$ is the spin operator for the nucleon, and $\hat{n}$ is the normal to the scattering plane. The coefficient $\alpha(k)$ is given in terms of the pion-nucleon phase shift $\delta$ by $\alpha(k)=k^{-1} \exp (i \delta) \sin \delta$. Only the operator $\vec{\sigma} \cdot \hat{n}$ can 
lead to a spin transfer $(\Delta S=1)$. Siciliano and Walker have shown that a similar expression holds for pion-nucleus scattering. ${ }^{5}$ At a momentum transfer $q \simeq q_{0}$, i.e., near the maximum of the differential cross section $\sigma(\theta)$ for pion-nucleus scattering,

$$
\sigma(\theta)=\Gamma(E)\left[4 M^{2}\left(q_{0}\right) \cos ^{2}(\theta)+S^{2}\left(q_{0}\right) \sin ^{2}(\theta)\right],
$$

if we assume dominance of the $[3,3]$ resonance, the validity of the fixed-scatterer impulse approximation and a one-step reaction mechanism. Here $E$ is the pion energy and $\theta$ is the scattering angle in the pion-nucleus laboratory frame. Both the spin-independent form factor $M\left(q_{0}\right)$ and the spin-dependent form factor $S\left(q_{0}\right)$ contribute to natural parity transitions, but these transitions are usually dominated by $M\left(q_{0}\right)$. Only $S\left(q_{0}\right)$ can contribute to $\Delta S=1$ transitions (if Fermi motion corrections are neglected). $\Gamma(E)$ contains the energy dependence of the pion-nucleon amplitudes, of the distortions, and the phase-space factors. ${ }^{5}$

At a constant momentum transfer $q$, the scattering angle $\theta$ is a simple function of the incident energy or wave number $k, \theta=2 \sin ^{-1}(q / 2 k)$. For example, to keep the momentum transfer constant at a $q$ value near the maximum in the angular distribution for a $\Delta L=2$ transition in ${ }^{13} \mathrm{C}$, the scattering angle must be decreased from about $72^{\circ}$ at $T_{\pi}=100 \mathrm{MeV}$ to about $32^{\circ}$ at $T_{\pi}=300 \mathrm{MeV}$. (Because of its large width the $[3,3]$ resonance is dominant over this whole energy range.) Thus the energy dependence of $\sigma(\theta)$ for $\Delta S=1$ transitions and $\Delta S=0$ transitions measured at constant momentum transfer will be very different simply because of the difference between the $\sin ^{2} \theta$ and $\cos ^{2} \theta$ functions. The energy dependences of the $\sin ^{2} \theta$ and $\cos ^{2} \theta$ functions are expected to dominate the shapes of the excitation functions since $\Gamma(E)$ depends only relatively weakly on the energy for constant $a$. Consequently, the cross sections for $\Delta S=1$ transitions should decrease with increasing energy since $\sin ^{2} \theta$ decreases between $100 \mathrm{MeV}\left(\theta \simeq 72^{\circ}\right)$ and $300 \mathrm{MeV}\left(\theta \simeq 32^{\circ}\right)$. In contrast, the cross sections for $\Delta S=0$ transitions should increase with increasing energy due to the associated $\cos ^{2} \theta$ dependence.

We have measured excitation functions for inelastic scattering of pions from ${ }^{13} \mathrm{C}$ at incident energies between 100 and $300 \mathrm{MeV}$ using the EPICS spectrometer at the Clinton $P$. Anderson Meson Physics Facility (LAMPF). The target was a $15 \mathrm{~cm} \times 23 \mathrm{~cm}$ sheet of carbon, of areal density $210 \mathrm{mg} / \mathrm{cm}^{2}$, enriched in ${ }^{13} \mathrm{C}$ to $99 \%$ and covered on both sides by thin $\left(\approx 1 \mathrm{mg} / \mathrm{cm}^{2}\right) \mathrm{Kap}-$ ton foils. The energy resolution width was about $250 \mathrm{keV}$ [full width at half maximum (FWHM)] (see Ref. 2 for typical pion spectra). At each energy the scattering angles were chosen to keep the momentum transfer fixed at either $1.1 \hbar \mathrm{fm}^{-1}$ or $1.4 \hbar \mathrm{fm}^{-1}$. The former value is close to the first maximum in the angular distribution for a $\Delta L=2$ transition and the latter is close to the maximum in the angular distribution for the strongly $\pi^{-}$-enhanced transition to a state at 9.5 $\mathrm{MeV} .^{2}$ At the smaller momentum transfer only $\pi^{+}$data were taken; at the larger momentum transfer both $\pi^{+}$and $\pi^{-}$data were taken and only the excitation function having the best statistics for each state (either $\pi^{+}$or $\pi^{-}$) is presented here. To determine the overall normalization of the $a b-$ solute cross sections, yields were nieasured for $\pi^{ \pm}+p$ as a function of energy at $\theta_{\mathrm{lab}}=40^{\circ}$ and compared with cross sections calculated with use of the pion-nucleon phase shifts of Rowe; Saloman, and Landau. ${ }^{7}$ The error in the normalization is estimated as $\pm 10 \% .^{8}$ This error is not included in the error bars of the figures which represent only the statistical and the fitting errors.

The $\left(\pi^{+}, \pi^{+}\right)$cross sections for the transitions to the strongly excited $\frac{3}{2}^{-}$state $(3.68 \mathrm{MeV})$ and the unresolved $\frac{5}{2}^{-}, \frac{7}{2}^{+}$doublet $(7.5 \mathrm{MeV})$ were measured at $q=1.1 \hbar \mathrm{fm}^{-1}$ and found to increase uniformly with energy (Fig. 1). The $\frac{3}{2}^{-}$and $\frac{5}{2}^{-}$. states are collectively enhanced and predicted to be dominated by the $\Delta L=2, \Delta S=0$ transition amplitude. ${ }^{9}$ At $q=1.1 \hbar \mathrm{fm}^{-1}$ the cross section measured for the doublet at $7.5 \mathrm{MeV}$ is expected to be due mostly to the $\frac{5}{2}^{-}$state and thus the excitation function for this group should show the features of a $\Delta S=0$ transition, like the excitation function for the transition to the $\frac{3}{2}^{-}$state. Indeed, for both states the simple $\cos ^{2} \theta$ relation (Fig. 1, solid lines) predicts the trend of the data quite well. A distorted-wave impulse approximation (DWIA) calculation of the $\frac{3}{2}^{-}$state with use of the code DWPI of Eisenstein and Miller ${ }^{10}$ with an "energyshifted" optical potential" and a simple collective form factor reproduces the shape of the excitation function in detail (Fig. 1, broken line). The relatively small differences between the $\cos ^{2} \theta$ function and the DWIA result verify the claims made above regarding the weakness of the energy dependence of $\Gamma$.

The $\left(\pi^{-}, \pi^{-\prime}\right)$ excitation function for the transition to the $9.5-\mathrm{MeV}$ state was measured at $q$ $=1.4 \hbar \mathrm{fm}^{-1}$ and found to decrease uniformly with energy (Fig. 2), in sharp contrast to the data for 


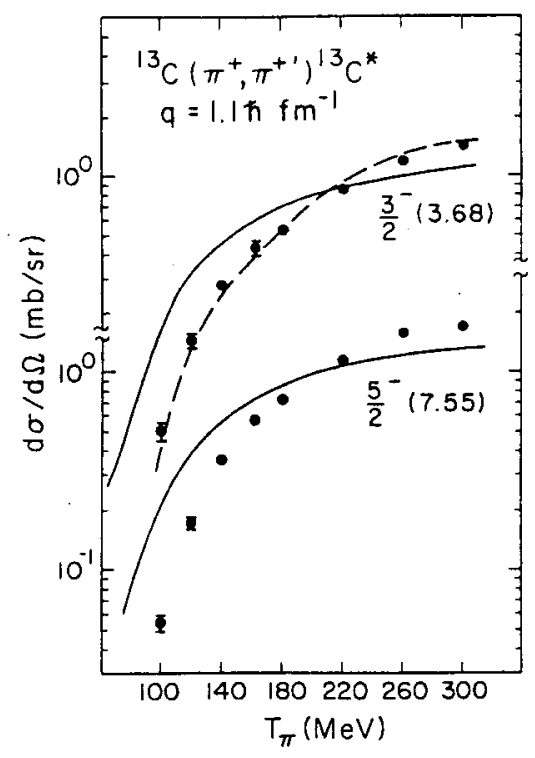

FIG. 1. Excitation functions for ${ }^{13} \mathrm{C}\left(\pi^{+}, \pi^{+1}\right)$ to the $\frac{3}{2}^{-}(3.68 \mathrm{MeV})$ and $\frac{5}{2}^{-}(7.55 \mathrm{MeV})$ states measured at $q=1.1 \hbar \mathrm{fm}^{-1}$. The solid lines are the $\cos ^{2} \theta$ dependences arbitrarily normalized to the data at $220 \mathrm{MeV}$. The dashed line is the result of a DWIA calculation for the $\frac{i^{-}}{2}$ state.

the $\Delta S=0$ transitions discussed above. The energy dependence is very well accounted for by $\sin ^{2} \theta$ (solid line).

It has been proposed that this state is the $\frac{9+}{2}$. member of a weak-coupling multiplet ${ }^{12}$ based on a $1 d_{5 / 2}$ neutron coupled to the first excited $2^{+}$ state in ${ }^{12} \mathrm{C}$. In this model the large $\pi^{-}$asymme$\operatorname{try}, A=\left[\sigma\left(\pi^{-}\right)-\sigma\left(\pi^{+}\right)\right] /\left[\sigma\left(\pi^{-}\right)+\sigma\left(\pi^{+}\right)\right]=0.8 \pm 0.2$, can be explained as due to a pure neutron $1 p_{3 / 2}$ $\rightarrow 1 d_{5 / 2}$ particle-hole excitation. ${ }^{2}$ The shell-model calculations of Millener and Kurath ${ }^{13}$ predict a $\frac{9+}{2}$ state at $9.5 \mathrm{MeV}$ which has a very large overlap with the weak-coupling state. The DWIA calculations of Lee and Kurath ${ }^{3}$ with use of the Mjllener-Kurath wave functions reproduce not onIy the $\pi^{-}$enhancement very well but also the absolute cross sections ${ }^{8}$ for exciting this state to within about $20 \%$. According to this model; the $\frac{9}{2}^{+}$ state is reached solely by an unnatural parity transition with a total-angular-momentum trans-. fer $\Delta J=4$, an orbital-angular-momentum transfer $\Delta L=3$, and a spin transfer $\Delta S=1$. The experimental excitation function for this state from the present work is characteristic of a $\Delta S=1$ transition, in excellent agreement with the shellmodel calculation. ${ }^{3,13}$

We remark that the enhancement of $\Delta S=1 \mathrm{tran}$ -

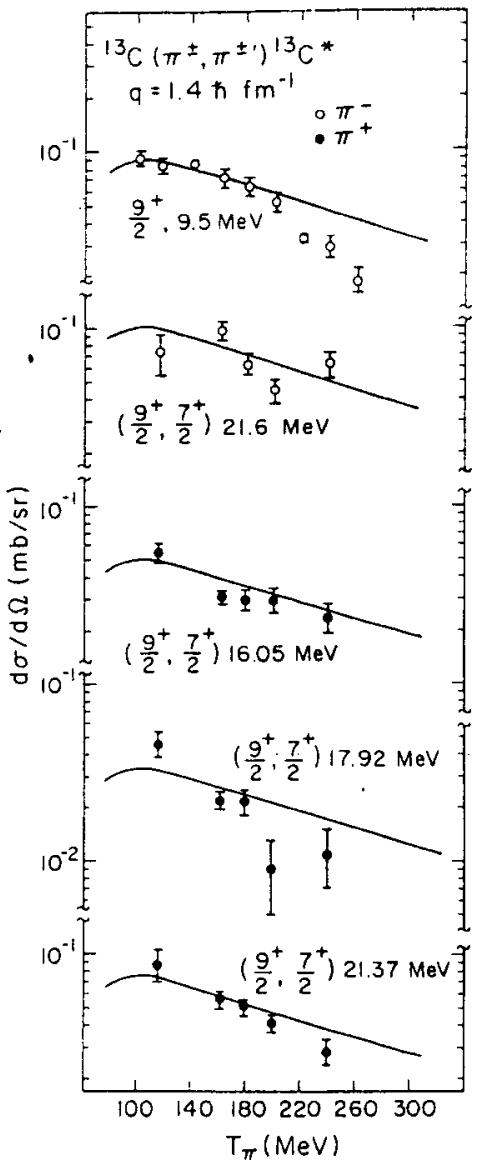

FIG. 2. Excitation functions for ${ }^{13} \mathrm{C}\left(\pi^{4}, \pi^{ \pm \prime}\right)$ to states at 9.5 and $21.60 \mathrm{MeV}$ (with $\pi^{-}$), and at $16.05,17.92$, and $21.37 \mathrm{MeV}$ (with $\pi^{+}$), measured at $q=1.4 \hbar \mathrm{fm}^{-1}$. The solid lines are simple $\sin ^{2} \theta$ dependences.

sitions relative to $\Delta S=0$ transitions at low pion energies $(\simeq 100 \mathrm{MeV})$ is of considerable help in searches for weak unnatural parity transitions. At or above resonance ( $>180 \mathrm{MeV}$ ) such transitions may be barely visible against a background of strong $\Delta S=0$ transitions.

Four states, or groups of states, in the excitation energy region from 12 to $24 \mathrm{MeV}$ were found to be strongly excited at large momentum transfer. Their angular distributions are fairly structureless and peak at large angles characteristic of a large angular-momentum transfer to highspin states. ${ }^{8}$ The centroids of these groups are at $16.05,17.92,21.37$, and $21.60 \mathrm{MeV}$. Their excitation functions measured at $q=1.4 \hbar \mathrm{fm}^{-1}$ are very similar to that of the $\frac{9}{2}^{+}$state at $9.5 \mathrm{MeV}$ and they are also very well described by $\sin ^{2} \theta$ (Fig. 2). Thus the transitions to these states must be dominated by $\Delta S=1$. The $16.05-\mathrm{MeV}$ 
state is $\pi^{+}$enhanced, $A=-0.6 \pm 0.2$ (Ref. 2), in agreement with the shell-model prediction ${ }^{3,13}$ for a $\frac{9+}{2}^{+}$state close to $16 \mathrm{MeV}$. The complex close to $21.5-\mathrm{MeV}$ peaks at $21.37 \mathrm{MeV}$ in $\pi^{+}$scattering and at $21.60 \mathrm{MeV}$ in $\pi^{-}$scattering. The state or group of states at $17.92 \mathrm{MeV}$ is excited about equally by both $\pi^{+}$and $\pi^{-}$. An interpretation of these results in terms of shell-model predictions is difficult because of the large number of states involved. The $21.5-\mathrm{MeV}$ complex probably contains several $\frac{7}{2}^{+}$and $\frac{y}{2}^{+}$states. Furthermore, the $T=\frac{1}{2}$ and $T=\frac{3}{2}$ states at these energies are most likely mixed in isospin. Isospin mixing was not included in Ref. 13 but its importance is suggested by the similarity of the pion spectra ${ }^{2}$ close to $21.5 \mathrm{MeV}$ in ${ }^{13} \mathrm{C}$ with those close to $19 \mathrm{MeV}$ in ${ }^{12} \mathrm{C}$ (Ref. 14).

The $180^{\circ}$ electron scattering data of Hicks et $a l .{ }^{15}$ and Dubach et al. ${ }^{16}$ are in agreement with our $\Delta S=1$ assignments for some of these transitions. They found $M 4$ transitions to states at $9.49,16.06$, and $21.43 \mathrm{MeV}$ but did not see any significant strength at $\simeq 17.9 \mathrm{MeV}$. A comparison of the different excitation strengths from $\left(\pi^{+}, \pi^{+1}\right),\left(\pi^{-}\right.$, $\left.\pi^{-1}\right)$, and $\left(e, e^{\prime}\right)$ allows conclusions about the isospin transfer $\Delta T$ since the transverse $\left(e, e^{\prime}\right)$ form factor is dominated by $\Delta T=1$. The near equality of $\sigma\left(\pi^{-}\right)$and $\sigma\left(\pi^{+}\right)$for the group at $17.92 \mathrm{MeV} \mathrm{sug-}$ gests a pure isospin transfer of either $\Delta T=0$ or $\Delta T=1$, but the lack of any significant $M 4$ strength from $\left(e, e^{\prime}\right)$ strongly supports a pure $\Delta T=0$ transition. The excitation energy of one of the strong $M 4$ transitions from $\left(e, e^{\prime}\right), E_{x}=21.43 \pm 0.03$ $\mathrm{MeV},{ }^{16}$ is close to the centroid of the peak seen in $\left(\pi^{+}, \pi^{+\prime}\right)$. This suggests a concentration of the $\Delta T=1$ strength in the lower-energy part of the 21.5-MeV complex.

In summary, these data have demonstrated the utility of measurements of excitation functions of inelastic pion scattering in the identification of $\Delta S=1$ transitions. This technique has been used to confirm that the pure transition to the $\frac{9}{2}^{+}$state at $9.5 \mathrm{MeV}$ involves $\Delta S=1$ and to identify four $\Delta S$ $=1$ transitions to states in the region from 12 to $24 \mathrm{MeV}$ in ${ }^{13} \mathrm{C}$. It is anticipated that this tech- nique can be used to separate the $\Delta S=0$ and $\Delta S$ $=1$ components of mixed transitions. This will allow stringent tests of shell-model predictions.

The authors are indebted to Dr. E. R. Siciliano, Dr. G. E. Walker, Dr. M. A. Franey, and Dr. T.S. H. Lee for helpful discussions and to Dr. R. L. Boudrie, Dr. G. Kyle, Dr. J. Piffaretti, and Dr. H. A. Thiessen for assistance in the early stages of data taking. The authors would also like to thank Dr. R. J. Macek for providing the ${ }^{13} \mathrm{C}$ target. This work was supported in part by the U. S. Department of Energy.

\footnotetext{
(a) Present address: Los Alamos National Laboratory, Los Alamos, N. Mex.

${ }^{1}$ C. Fred Moore et al ., Phys. Lett. 80B, 38 (1978).

${ }^{2} \mathrm{D}$. Dehnhard et al., Phys. Rev. Lett. 43, 1091 (1979).

${ }^{3}$ T.-S. H. Lee and D. Kurath, Phys. Rev. C 22,1670 (1980).

${ }^{4}$ W. Cottingame et al ., Bull. Am. Phys. Soc. 24, 821 (1979).

${ }^{5}$ E. R. Siciliano and G. E. Walker, Phys. Rev. C (to be published).

${ }^{6} \mathrm{D}$. S. Koltun, in Advances in Nuclear Science, edited by M. Baranger and E. Vogt (Plenum, New York, 1969), Vol. 3, pp. 71, ff.

${ }^{7}$ G. Rowe, M. Saloman, and R. H. Landau, Phys.

Rev. C 18, 384 (1978).

${ }^{8}$ S. J. Seestrom-Morris, Ph.D. dissertation, University of Minnesota, 1981 (unpublished).

${ }^{9}$ T.-S. H. Lee and D. Kurath, Phys. Rev. C 21, 293 (1980).

${ }^{10}$ R. A. Eisenstein and G. A. Miller, Comput. Phys. Commun. 11, 95 (1976).

${ }^{11}$ W. B. Cottingame and D. B. Holtkamp, Phys. Rev. Lett. 45,1828 (1980).

${ }^{12}$ A. M. Lane, Rev. Mod. Phys. 32, 519 (1960).

${ }^{13}$ D. J. Millener and D. Kurath, Nucl. Phys. A255, 315 (1975); D. Kurath, private communication.

${ }^{14}$ C. L. Morris et al ., Phys. Lett. 86B, 31 (1979).

${ }^{15}$ R. S. Hicks ot al., in Proceedings of the Workshop on Nuclear Structure with Intermediate Energy Probes, Los Alamos, New Mexico, 1980, edited by H. A. Thiessen (unpublished), p. 292, LAMPF Report No. LA8303-C, 1980.

${ }^{16} \mathrm{~J}$. Dubach et al ., to be published.
} 


\footnotetext{
${ }^{15}$ This assumes, of course, the $c \rightarrow s$ transition to be dominant. If on the other hand the higher KobayashiMaskawa angles are large, the $c \rightarrow b$ transition would be enhanced at the cost of $c \rightarrow s$; and the $5 \%$ rise from in-
}

trinsic charm would be spread over even a wider range of energy.

${ }^{16}$ V. Barger, W. Y. Keung, and R. J. N. Phillips, Phys. Rev. D 24, 244 (1981).

\title{
Strong Cancellations of Neutron and Proton Transition Amplitudes Observed in Pion Inelastic Scattering from ${ }^{14} \mathrm{C}$
}

D. B. Holtkamp, S. J. Seestrom-Morris, S. Chakravarti, and D. Dehnhard University of Minnesota, Minneapolis, Minnesota 5.54 .55

and

Helmut W. Baer and C. L. Morris

Los Alamos National Laboratory, Los Alamos, New Mexico 87545

and

S. J. Greene

New Mexico State University, Las Cruces, New Mexico 8800.3

and

\author{
Carol J. Harvey \\ University of Texas, Austin, Texas 78712
}

(Received 24 April 1981)

\begin{abstract}
Differential cross sections were measured for inelastic scattering of $164-\mathrm{MeV} \pi^{+}$and $\pi^{-}$from ${ }^{14} \mathrm{C}$. Large cross-section ratios, $R=\sigma\left(\pi^{+}\right) / \sigma\left(\pi^{-}\right)$(or $R^{-1}$ ), were observed, two being significantly larger than the free $\pi$-nucleon value. Lower limits are $R>26, R^{-1}$ $>17$, and $R>11$ for states at $8.32 \mathrm{MeV}\left(2^{+}\right), 11.67 \mathrm{MeV}\left(4^{-}\right)$, and $17.26 \mathrm{MeV}\left(4^{-}\right)$, respectively. These values are interpreted as being due to strong cancellations of the neutron and proton components of the transition amplitudes.
\end{abstract}

PACS numbers: $25.80 .+\mathrm{f}, 21.10 . \mathrm{Hw}$

At energies near the $(3,3) \pi-N$ (pion-nucleon) resonance, the ratio, $R=\sigma\left(\pi^{+}\right) / \sigma\left(\pi^{-}\right)$, of the cross sections for $\pi^{+}$and $\pi^{-}$scattering from free protons (neutrons) is about $9\left(\frac{1}{9}\right)$. Thus, comparisons of $\pi^{+}$and $\pi^{-}$inelastic scattering from nuclei should provide a sensitive method of separating the proton and neutron components of inelastic transitions. Cross-section ratios near the free $\pi-N$ values are expected for pure proton or pure neutron particle-hole $(p-h)$ excitations if the impulse approximation is valid. However, in previous studies on even- $A, N>Z$ nuclei, such as ${ }^{18} \mathrm{O}$ (Ref. 1) and ${ }^{26} \mathrm{Mg}$ (Ref. 2), values of $R$ (or $R^{-1}$ ) in the range of 1.0 to 2.0 were found. These small values have been attributed to the large amount of core excitation present in these nuclei. Deviations from $R=1$ for $N=Z$ nuclei, which arise from charge symmetry violation (e.g., due to the Coulomb force), have been observed and interpreted successfully as due to transitions to an isospin-mixed doublet in ${ }^{12} \mathrm{C}$ (Ref. 3) and a triplet in ${ }^{16} \mathrm{O}$ (Ref. 4). The free $\pi$-neutron value of $R^{-1} \simeq 9$ was detected ${ }^{5}$ so far only for a transition in the odd $-A$ nucleus ${ }^{13} \mathrm{C}$ and was irterpreted as the result of a pure neutron $\mathrm{p}-\mathrm{h}$ transition to a "stretched" state.

In this Letter, we report on the first observation of ratios $R$ significantly larger than 9 in a measurement of inelastic pion scattering on ${ }^{14} \mathrm{C}$ - at an incident energy $T_{n}=164 \mathrm{MeV}$. The large differences in $\pi^{+}$and $\pi^{-}$cross sections are seen to provide unusually transparent signatures of the shell-model structure of the transitions. As discussed in detail below, the near vanishing of either $\pi^{+}$or $\pi^{-}$cross sections (but not both) for a specific state comes about from the strong cancellation of the proton and neutron transition amplitudes.

The experiment was performed at the Clinton P. Anderson Meson Physics Facility with the 


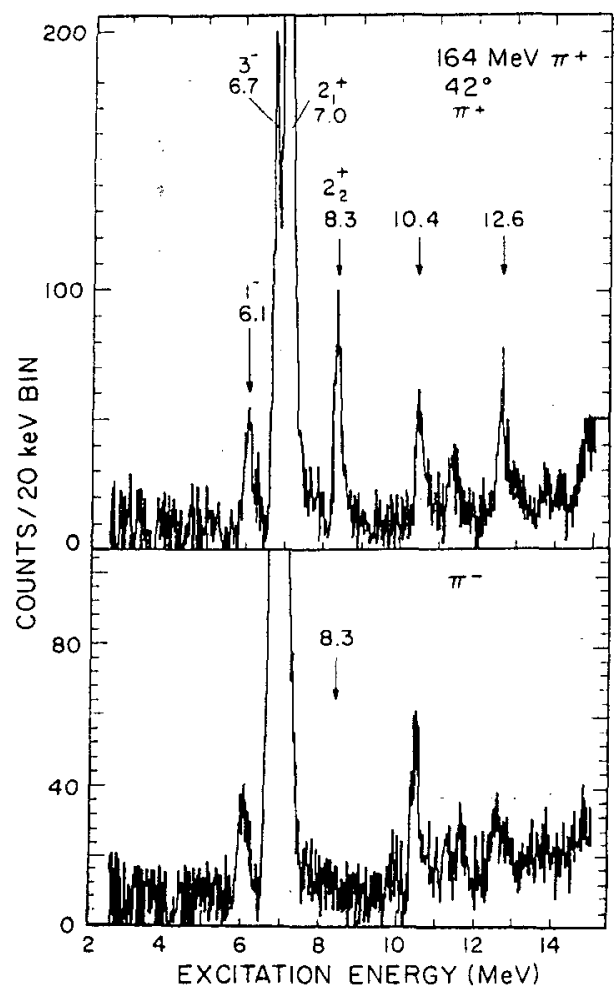

FIG. 1. Energy loss spectra for $\pi^{+}$and $\pi^{-}$scattering from ${ }^{14} \mathrm{C}$ at $\theta_{\mathrm{lab}}=42^{\circ}$ and $T_{\pi}=164 \mathrm{MeV}$.

energetic pion channel and spectrometer which has been described in detail elsewhere. ${ }^{6}$ The target consisted of $\sim 9 \mathrm{~g}$ of carbon powder enriched to $83 \%$ in ${ }^{14} \mathrm{C}$. The ${ }^{14} \mathrm{C} /{ }^{12} \mathrm{C}$ atom ratio was 4.87 \pm 0.24 . ${ }^{7}$ The carbon powder was pressed to a thickness of $\sim 0.10 \mathrm{~g} / \mathrm{cm}^{2}$ into six target cells, each consisting of a U-shaped stainless-steel frame covered with $0.025-\mathrm{mm}$-thick windows made of stainless steel. Since the ${ }^{14} \mathrm{C}$ target was smaller than the beam spot size, strips of natural graphite were placed above and below the ${ }^{14} \mathrm{C}$ target cells in order to obtain ${ }^{12} \mathrm{C}$ and ${ }^{14} \mathrm{C}$ spectra simultaneously. Additional spectra were taken from stainless-steel foils as target. These spectra enabled us to derive "clean" spectra via a channel-by-channel subtraction, shown in Figs. 1 and 2. The energy resolution obtained was about $200 \mathrm{keV}$ (full width at half maximum).

Cross sections at $42^{\circ}$ and $66^{\circ}$ for selected states are presented in Table $\mathrm{I}$. The ${ }^{14} \mathrm{C}$ cross sections were normalized with use of the $\pi^{*}$ cross sections for the $4.44-\mathrm{MeV}$ state in ${ }^{12} \mathrm{C}$, the ${ }^{12} \mathrm{C}(4.44 \mathrm{MeV})$ yield from the ${ }^{14} \mathrm{C}$ target, and the known ${ }^{14} \mathrm{C} /{ }^{12} \mathrm{C}$ atom ratio. We employed this method of normalization since it does not rely on the assumption

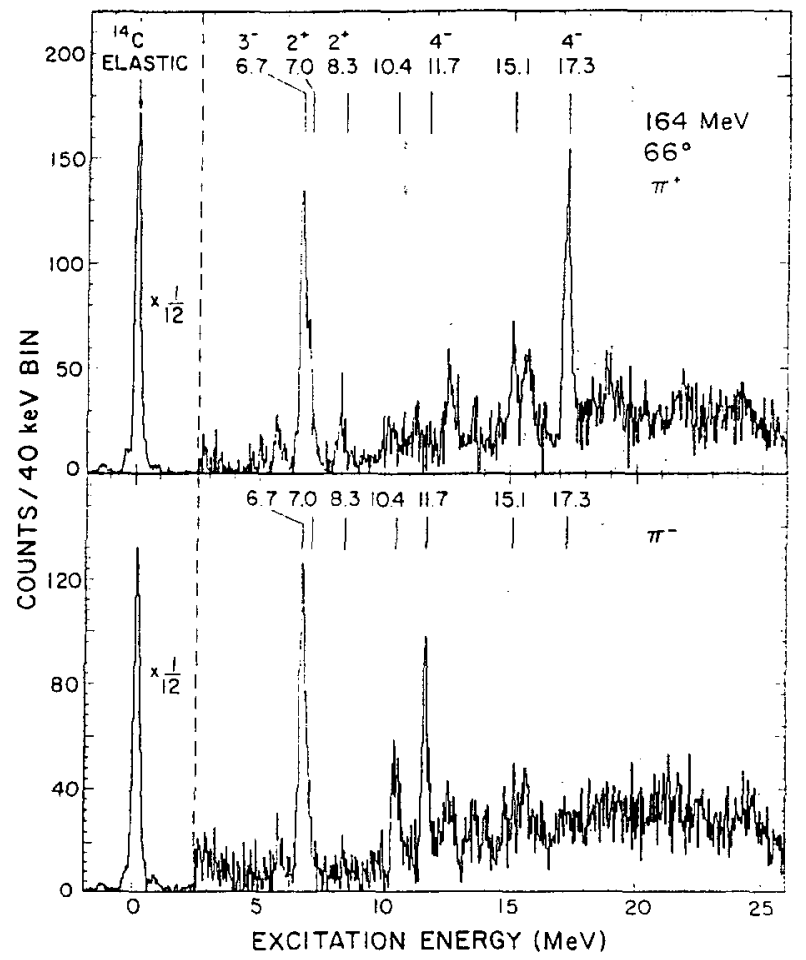

FIG. 2. Same as Fig. 1, but at $66^{\circ}$.

of uniform target thickness. The absolute normalization was obtained by comparing $\pi^{ \pm}$-hydrogen yields with the $\pi$-proton cross sections of Dodder. ${ }^{8}$ The ${ }^{12} \mathrm{C}$ cross sections (Table I), which were measured using the graphite strips, are in good agreement with previous work. ${ }^{9}$

The most surprising feature of the present data is the sharp contrast between the near equality of $\sigma\left(\pi^{+}\right)$and $\sigma\left(\pi^{-}\right)$for the $2_{1}^{+}$state at $7.01 \mathrm{MeV}$ and the highly asymmetric cross sections for the $2_{2}{ }^{+}$ state at $8.32 \mathrm{MeV}$. At $\theta_{1 \mathrm{ab}}=42^{\circ}$, which is near the first maximum of known $\Delta l=2$ angular dis tributions, no $\pi^{-}$yield could be detected above background (Fig. 1). The experimental cross sections and errors (Table $I$ ) yield a lower limit of $R>26$ for the $2_{2}{ }^{+}$state. This is dramatically larger than the free $\pi$-proton value of 9 .

Similarly, at $\theta_{1 \mathrm{ab}}=66^{\circ}$ (Fig. 2), a state at 11.67 $\mathrm{MeV}$ is observed only in $\left(\pi^{-}, \pi^{-\prime}\right)$ and a state at $17.26 \mathrm{MeV}$ is seen essentially only in $\left(\pi^{+}, \pi^{+}\right)$. The lower limits for the cross-section ratios are $R^{-1}>17$ and $R>11$, respectively. Angular distributions for these states (not shown) have maxima near $66^{\circ}$ and shapes similar to those of previously measured $\Delta J=4$ transitions in ${ }^{12} \mathrm{C}$ (Ref, 3 ), ${ }^{13} \mathrm{C}$ (Ref. 5), and ${ }^{16} \mathrm{O}$ (Ref. 4), all of which are due to excitations of the "stretched" $\left(d_{5 / 2}, p_{3 / 2}{ }^{-1}\right)$ 
TABLE I. The measured $\pi^{+}$and $\pi^{-}$differential cross sections (lab), ${ }^{a}$ cross-section ratios $R$, and asymmetries $A$ b for states in ${ }^{14} \mathrm{C}$ and the $4.44-\mathrm{MeV}$ state in ${ }^{12} \mathrm{C}$.

\begin{tabular}{lccccccc}
\hline Target & $\begin{array}{c}E_{x} \\
(\mathrm{MeV})\end{array}$ & $J^{\pi}$ & $\begin{array}{c}\theta_{\mathrm{lab}} \\
(\mathrm{deg})\end{array}$ & $\begin{array}{c}d \sigma / d \Omega_{\pi}^{+} \\
(\mathrm{mb} / \mathrm{sr})\end{array}$ & $\begin{array}{c}d \sigma / d \Omega_{\pi}^{-} \\
(\mathrm{mb} / \mathrm{sr})\end{array}$ & $R$ & $A$ \\
\hline${ }^{14} \mathrm{C}$ & 7.01 & $2^{+}$ & $42^{\circ}$ & $1.01 \pm 0.08$ & $0.90 \pm 0.07$ & $1.12 \pm 0.07$ & $-0.06 \pm 0.03$ \\
${ }^{14} \mathrm{C}$ & 8.32 & $2^{+}$ & $42^{\circ}$ & $0.14 \pm 0.01$ & $<0.0054^{\mathrm{c}}$ & $>26^{\mathrm{c}}$ & $-0.99 \pm 0.09$ \\
${ }^{14} \mathrm{C}$ & $11.67^{\mathrm{d}}$ & ${\left(4^{-}\right)}^{\circ}$ & $66^{\circ}$ & $<0.0028^{\mathrm{c}}$ & $0.049 \pm 0.005$ & $<1 / 17^{\mathrm{c}}$ & $0.96 \pm 0.04$ \\
${ }^{14} \mathrm{C}$ & $17.26^{\mathrm{d}}$ & \left.${\left(4^{-}\right)}^{-}\right)$ & $66^{\circ}$ & $0.094 \pm 0.008$ & $0.005 \pm 0.003$ & $>11^{\mathrm{c}}$ & $-0.89 \pm 0.06$ \\
${ }^{12} \mathrm{C}$ & 4.44 & $2^{+}$ & $42^{\circ}$ & $2.85 \pm 0.17$ & $2.88 \pm 0.18$ & $0.99 \pm 0.06$ & $0.006 \pm 0.03$ \\
\hline
\end{tabular}

${ }^{a}$ The errors quoted are based on an estimate of all known uncertainties but do not include $a \pm 20 \%$ error in the overall normalization. In calculating $R$ and $A$, the cancellation of some errors which are common to $\pi^{+}$and $\pi^{-}$was taken into account.

${ }^{\mathrm{b}} A \equiv\left[\sigma\left(\pi^{-}\right)-\sigma\left(\pi^{+}\right)\right] /\left[\sigma\left(\pi^{-}\right)+\sigma\left(\pi^{+}\right)\right]$.

${ }^{c}$ At the $90 \%$ confidence level.

${ }^{d}$ The estimated uncertainty in the energies of these states is $\pm 0.1 \mathrm{MeV}$.

$\mathrm{p}-\mathrm{h}$ configuration. On the basis of this similarity to previous pion data, we suggest a $J^{\pi}$ assignment of $4^{-}$for these states.

It has been known for some time that the $p$-shell model ${ }^{10}$ predicts only one low-lying $2^{+}$state, yet the two experimental $2^{+}$states at 7.01 and 8.32 $\mathrm{MeV}$ share the $p$-shell strength for transfer. reac:tions, ${ }^{11}$ electromagnetic transitions, ${ }^{11}$ and the $\left(\pi^{-}, \gamma\right)$ reaction. ${ }^{12}$ On the basis of previous work $^{13,14}$ a reasonable approximation to the wave functions of the two $2^{+}$states $(i=1,2)$ is

$$
\begin{aligned}
\mid{ }^{14} \mathrm{C} ; 2_{i}{ }^{+}, T & =1\rangle \\
& \left.=\alpha_{i}\left|\left(p^{-2 p}\right)_{2+}\right\rangle+\beta_{i} \mid\left(p^{-4}\right)_{0+}\left(s d^{2 \pi}\right)_{2+}\right),
\end{aligned}
$$

with $\alpha_{1}=\alpha_{2}=1 / \sqrt{2}$ and $\beta_{1}=-\beta_{2}=-1 / \sqrt{2}$, and for the ground state (g.s.)

$$
\begin{aligned}
& \left|{ }^{14} \mathrm{C} 0_{1}{ }^{+}, T=1\right\rangle \\
& \quad=\alpha_{0}\left|\left(p^{-2 p}\right)_{0+}\right\rangle+\beta_{0}\left|\left(p^{-4}\right)_{0+}\left(s d^{2 n}\right)_{0+}\right\rangle .
\end{aligned}
$$

The relative phases of the $p$-shell (proton) and $s-d$-shell (neutron) parts of the $2^{+}$states indicate constructive interference of the proton and neutron components in the transition from the g.s. to the $2_{1}{ }^{+}$state and destructive interference for the $2_{2}{ }^{+}$state. This destructive interference could result in a near vanishing of $\sigma\left(\pi^{-}, 2_{2}{ }^{+}\right)$even if $\beta_{0}$ is relatively small because of the enhancement of the neutron part of the transition amplitude in $\pi^{-}$scattering.

To prove this point quantitatively, we performed momentum-space distorted-wave impulse-approximation ${ }^{15}$ calculations. The $\mathrm{p}-\mathrm{h}$ matrix elements for the $p$-shell proton holes and the $s-d-$ shell neutron particles were obtained from the work of Ellis and Engeland ${ }^{16}$ and the coefficient $\alpha_{0}\left[\beta_{0}\right.$ $\left.=-\left(1-\alpha_{0}{ }^{2}\right)^{1 / 2}\right]$ was taken as an adjustable parameter. Harmonic-oscillator radial wave functions were used for the bound states of the active nucleons with an oscillator parameter $b=1.7 \mathrm{fm}$ for both protons and neutrons. ${ }^{13}$ Both spin transfer values of $\Delta S=0$ and $\Delta S=1$ were included in calculating the scattering amplitudes. To account for the truncation of the model space, effective charges for both protons and neutrons were used to enhance the $\Delta S=0$ piece of the nuclear transition matrix elements. ${ }^{15}$ The polarization charges for the protons and neutrons were set equal $\left(\delta_{D}\right.$ $=\delta_{n}$ ) and treated as a second adjustable parameter. Further details of the calculation will be presented in a subsequent publication.

With $\delta_{p}=\delta_{n}=0.4, \alpha_{0}=0.81$, and $\beta_{0}=-0.19$, the calculations yield (in $\mathrm{mb} / \mathrm{sr}$ at $\left.42^{\circ}\right) \sigma\left(\pi^{+}, 2_{1}{ }^{+}\right.$) $=1.04, \sigma\left(\pi^{-}, 2_{1}{ }^{+}\right)=0.80, \sigma\left(\pi^{+}, 2_{2}{ }^{+}\right)=0.21$, and $\sigma\left(\pi^{-}, 2_{2}{ }^{+}\right)=0.008$. The data indicate an even more complete cancellation for the $2_{2}{ }^{+}$than these calculations do. Possible reasons for the remaining discrepancies are presently being investigated. Nevertheless, the basic features of the data are correctly described as due to an interference of the neutron and proton parts of the transition amplitude.

The data for the two proposed $4^{-}$states at 11.67 and $17.26 \mathrm{MeV}$ are also suggestive of cancellation effects. In any simple model, several $4^{-}, T$ $=1$ states are expected with large components of the "stretched" $\left(d_{5 / 2}, p_{3 / 2}{ }^{-1}\right)$ configuration. The $4^{-}$state lowest in energy should have a large 
overlap with a state formed by a $d_{5 / 2}$ neutron coupled to a $\frac{3^{-}}{2}, T=\frac{1}{2}{ }^{13} \mathrm{C}$ core. ${ }^{13}$ This state would be reached mainly by a neutron $\mathrm{p}-\mathrm{h}$ excitation from a $T=\frac{1}{2}$ core component in the ground state of ${ }^{14} \mathrm{C}$. The experimental ratio for the $4_{1}{ }^{-}$state at $11.67 \mathrm{MeV}, R^{-1}>17$, is larger than the free $\pi$-neutron value of $R^{-1} \simeq 9$, indicating the presence of small contributions from proton $\mathrm{p}-\mathrm{h}$ excitations to the transition density amplitude with a sign opposite to the one of the principal neutron $\mathrm{p}-\mathrm{h}$ excitation.

Although the lower limit of $R>11$ for the other prominent $4^{-}$state at $17.26 \mathrm{MeV}$ is essentially consistent with a pure proton excitation $(R \simeq 9)$, such an interpretation is unlikely since it would imply a transition to a state strongly mixed in isospin. Cross-section ratios estimated from a shell-model calculation by Kurath ${ }^{17}$ for $4^{-}$model states at 12.7 and $16.9 \mathrm{MeV}$ are in good agreement with our data, although the estimated absolute cross sections are about a factor of 2 too large. A complete comparison of all the data with these predictions will be presented in a forthcoming publication.

In summary, our measurements on ${ }^{14} \mathrm{C}$ have shown for the first time that $\pi^{+} / \pi^{-}$cross-section ratios for nuclear excitations can be considerably larger than the value of 9 for scattering from free nucleons at the $(3,3)$ resonance. In addition, candidates for the first $4^{-}$states of "stretched" $\left(d_{5 / 2}, p_{3 / 2}{ }^{-1}\right)$ configuration in ${ }^{14} \mathrm{C}$ have been located. We have interpreted the extraordinarily large $\pi^{+} /$ $\pi^{-}$ratios as being due to cancellations of the neutron and proton components of the transition amplitudes. The direct manifestation of such cancellation phenomena in a simple comparison of the measured $\pi^{+}$and $\pi^{-}$spectra demonstrates the value of pion scattering for elucidating the structure of nuclear states.

Discussions with P. J. Ellis, H. T. Fortune, M. A. Franey, D. Kurath, and C. Fred Moore are gratefully acknowledged. The authors also thank D. Kurath for making his calculations for the negative-parity states available to us prior to the experiment.

This work was supported in part by the U. S. Department of Energy and the Robert A. Welch Foundation.

${ }^{1}$ S. Iverson et al., Phys. Lett. $82 \mathrm{~B}, 51$ (1979).

${ }^{2} \mathrm{C}$. A. Wiedner et al., Phys. Lett. 78B, 201 (1978).

${ }^{3}$ C. L. Morris et al., Phys. Lett. 86B, 31 (1979).

${ }^{4}$ D. B. Holtkamp et al., Phys. Rev. Lett. 45,420 (1980).

${ }^{5}$ D. Dehnhard et al., Phys. Rev. Lett. 43, 1091 (1979).

${ }^{6}$ H. A. Thiessen and S. Sobottka, Los Alamos Scientific Laboratory Report No. LA-4534-MS (unpublished).

${ }^{7}$ Taken from target assay provided by Oak Ridge National Laboratory.

${ }^{8}$ D. Dođder, private communication.

${ }^{9} \mathrm{~J}$. Piffaretti et al., Phys. Lett. 71B, 324 (1977); B. Chabloz et al., Phys. Lett. 81B, 143 (1979).

${ }^{10} \mathrm{~S}$. Cohen and D. Kurath, Nucl: Phys. 73,1 (1965).

${ }^{11}$ F. Ajzenberg-Selove, Nucl. Phys. A2 $\overline{68}, 1$ (1976), and to be published.

${ }^{12} \mathrm{H}$. W. Baer et al., Phys. Rev. C 12, 921 (1975).

${ }^{13}$ S. Lie, Nucl. Phys. A181, 517 (1972).

${ }^{14}$ N. F. Mangleson et al., Nucl. Phys. A117, 161

(1968); H. T. Fortune, private communication.

${ }^{15}$ T. -S. H. Lee and D. Kurath, Phys. Rev. C 21, 293 (1980).

${ }^{16}$ P. J. Ellis and T. Engeland, Nucl. Phys. Al 44,161 (1.970).

${ }^{17} \mathrm{D}$. Kurath, private communication. 


\title{
Measurement of Isospin Mixing in ${ }^{4} \mathrm{He}$ and Its Implications for Charge-Symmetry Breaking
}

\author{
C. L. Blilie, D. Dehnhard, D. B. Holtkamp, ${ }^{(a)}$ S. J. Seestrom-Morris, and S. K. Nanda \\ School of Physics and Astronomy, University of Minnesota, Minneapolis, Minnesota 55455 \\ W. B. Cottingame ${ }^{(a)}$ \\ Department of Physics, New Mexico State University, Las Cruces, New Mexico 88003 \\ D. Halderson \\ Department of Physics, Western Michigan University, Kalamazoo, Michigan 49008
}

C. L. Morris

Los Alamos National Laboraiory, Los Alamos, New Mexico 87545

C. Fred Moore and P. A. Seidl

Department of Physics, University of Texas, Austin, Texas 78712

H. Ohnuma

Department of Physics, Tokyo Institute of Technology, Oh-Okayama, Meguro, Tokyo 152, Japan

and

\author{
K. Maeda \\ College of General Education, Tohoku University, Kawauchi, Sendai 980, Japan
}

(Received 10 April 1985)

\begin{abstract}
$\pi{ }^{4} \mathrm{He}$ inelastic cross sections have been measured at $T_{\pi}=180 \mathrm{MeV}$. In the region of the $J^{\pi}=1^{-}$states $\left(E_{x} \simeq 23\right.$ to $\left.30 \mathrm{MeV}\right)$ the $\pi^{+} / \pi^{-}$cross-section ratio $R_{\pi}$ was found to be $1.05 \pm 0.08$ at $\theta_{\text {lab }}=30^{\circ}$. Such a small deviation from 1.0 implies that isospin mixing between the $T=0$ and $T=1$ states in ${ }^{4} \mathrm{He}$ is quite weak, in striking contrast to the strong isospin mixing deduced from the large ratio of photonucleon cross sections, $R_{\gamma}=\sigma(\gamma, p) / \sigma(\gamma, n)=1$.7. Thus the conclusion drawn from the photonucleon work, that there is a large charge-symmetry-breaking nuclear force in ${ }^{4} \mathrm{He}$, needs to be reexamined.
\end{abstract}

PACS numbers: $25.80 . \mathrm{Fm}, 25.10 .+\mathrm{s}, 27.10 .+\mathrm{h}$

A recent critical review ${ }^{1}$ of the numerous measurements ${ }^{2}$ of the $(\gamma, p)$ and $(\gamma, n)$ photonucleon cross sections on ${ }^{4} \mathrm{He}$ presents a cross-section ratio $R_{\gamma} \equiv \sigma(\gamma, p) / \sigma(\gamma, n)$ between 1.5 and 1.9 at excitation energies $E_{x}$ below $30 \mathrm{MeV}$. Measurements of the cross sections for the proton ${ }^{3}$ and neutron ${ }^{4}$ capture reactions, ${ }^{3} \mathrm{H}(p, \gamma)$ and ${ }^{3} \mathrm{He}(n, \gamma)$, confirm the $(\gamma, p)$ and $(\gamma, n)$ results. The experimental value of $R_{y}-1.7$ is in striking contrast to the value of 1.0 which would be expected if the proton-proton and neutron-neutron forces were equal so that the excited states of ${ }^{4} \mathrm{He}$ would be states of good isospin $T$. The $\gamma$ radiation in the photonucleon reactions involves predominantly electric dipole radiation $(E 1)$. Thus the large value of $R_{\gamma}$ may be due to isospin mixing between the $J^{\pi}=1^{-}, T=0$, and $T=1$ states in ${ }^{4} \mathrm{He}$ (Ref. 1). Because Coulomb effects can cause $R_{y}$ to be: only slightly larger $(=10 \%)$ than 1.0 at 2 to $3 \mathrm{MeV}$ above the $p+{ }^{3} \mathrm{H}$ threshold $(19.82 \mathrm{MeV}),{ }^{5-7}$ the au. thors of Ref. 1 suggested the presence of a large charge-symmetry-breaking (CSB) nuclear force in ${ }^{4} \mathrm{He}$ which would cause strong isospin mixing between the $1^{-}$states. An analysis ${ }^{1}$ of the photonucleon data with the two-level isospin-mixing formula of Barker and Mann ${ }^{8}$ yielded a CSB matrix element $\left\langle T=1\left|H_{\mathrm{CSB}}\right| T\right.$ $=0\rangle$ of nearly $500 \mathrm{keV}$; i.e., much larger than the value expected from the Coulomb force $(\simeq 100$ to $150 \mathrm{keV})$. An, even larger matrix element $(=2.5$ $\mathrm{MeV}$ ) was extracted ${ }^{9}$ in a calculation which included $3 \hbar \omega$ excitations. The existence of large CSB forces such as invoked in Refs. 1 and 9 would fundamentally change our present understanding of the nuclear force.

It has been shown ${ }^{10,11}$ that CSB forces of the magnitude required to reproduce the experimental value of $R_{\gamma}$ generate large differences between the polarization $(P)$ and the analyzing power $(A)$ in the reaction ${ }^{3} \mathrm{H}(p, n)^{3} \mathrm{He}$. Experimentally, ${ }^{12,13}$ however, only small differences have been observed between $A$ and $P$, in very good agreement with theoretical predictions ${ }^{11}$ which include only the Coulomb interaction as a CSB 
force. It is therefore necessary to measure the degree of isospin mixing by another method.

Inelastic scattering of $\pi^{+}$and $\pi^{-}$near the $[3,3]$ resonance has been established ${ }^{14,15}$ to be a reliable method for the determination of isospin mixing. For the $J^{\pi}=1^{+}$isospin doublet in ${ }^{12} \mathrm{C}(12.71 \mathrm{MeV}, T=0$, and $15.11 \mathrm{MeV}, T=1)$ the CSB matrix element has been found ${ }^{14}$ to be $=150 \mathrm{keV}$. This result agrees very well with electromagnetic measurements and theoretical expectations for isospin mixing due to the Coulomb force. Thus, in order to obtain an independent determination of isospin mixing in ${ }^{4} \mathrm{He}$, we have measured differential cross sections $\sigma\left(\pi^{+}\right)$and $\sigma\left(\pi^{-}\right)$for inelastic pion scattering from ${ }^{4} \mathrm{He}$ at $T_{\pi}=180 \mathrm{MeV}$.

The energetic pion channel and spectrometer system (EPICS) ${ }^{16}$ at the Los Alamos Meson Physics Facility (LAMPF) was used to measure spectra at $\theta_{\mathrm{lab}}=20^{\circ}$, $30^{\circ}$, and $40^{\circ}$ up to $E_{x}=45 \mathrm{MeV}$ excitation energy. The target was helium gas, cooled to a temperature $T=17 \mathrm{~K}$ at a pressure $P=2 \mathrm{~atm}$. The target container was a cylinder of $0.23-\mathrm{m}$ height and $0.127-\mathrm{m}$ diameter with nickel walls of $40-\mathrm{mg} / \mathrm{cm}^{2}$ areal density. The areal density of the cooled helium was $\simeq 65$ $\mathrm{mg} / \mathrm{cm}^{2}$. The energy resolution was $=500 \mathrm{keV}$ (FWHM) and the angular acceptance was $3^{\circ}$.

The absolute differential cross sections were determined by a measurement of $\pi$-hydrogen yields, with methane as a target, and normalizing the yields to $\pi+p$ cross sections, calculated from the phase shifts of Thiessen and Sobottka. ${ }^{17}$ We also measured spectra from the empty target container to determine the background contribution from the target can. For $\theta_{\text {lab }}=30^{\circ}$ the background amounted to about $15 \%$ of the total counts.

The experimental spectra at $\theta_{\text {lab }}=30^{\circ}$ (after background subtraction) were converted to differential cross sections $\sigma(\pi) \equiv d^{2} \sigma / d \Omega d E$. The results are shown in Fig. 1 for $E_{x} \leqslant 36 \mathrm{MeV}$. The error bars are due to statistics and the uncertainties in background subtraction only. Uncertainties in target thicknesses, pion decay fractions, wire-chamber efficiencies, computer live time, spectrometer acceptance, beam monitoring, and predicted $\pi+p$ cross sections lead to a conservative estimate of $\pm 15 \%$ uncertainty in the absolute differential cross sections.

In contrast to the photonucleon reactions, which are dominated by electric dipole absorption, inelastic scattering by pions excites all the states in ${ }^{4} \mathrm{He}$, except for the $J^{\pi}=0^{-}$state. All excited states of ${ }^{4} \mathrm{He}$ are particle unbound. Thus the spectra show a broad continuum of states above threshold $(\simeq 20 \mathrm{MeV})$. Nevertheless, the first excited $0^{+}$state at $20.5 \mathrm{MeV}$ and the $2^{-}$ state near $22 \mathrm{MeV}$ are clearly visible in Fig. 1. As indicated by the theoretical curves (to be discussed below), the $1^{-}$continuum peaks near $25 \mathrm{MeV}$. At
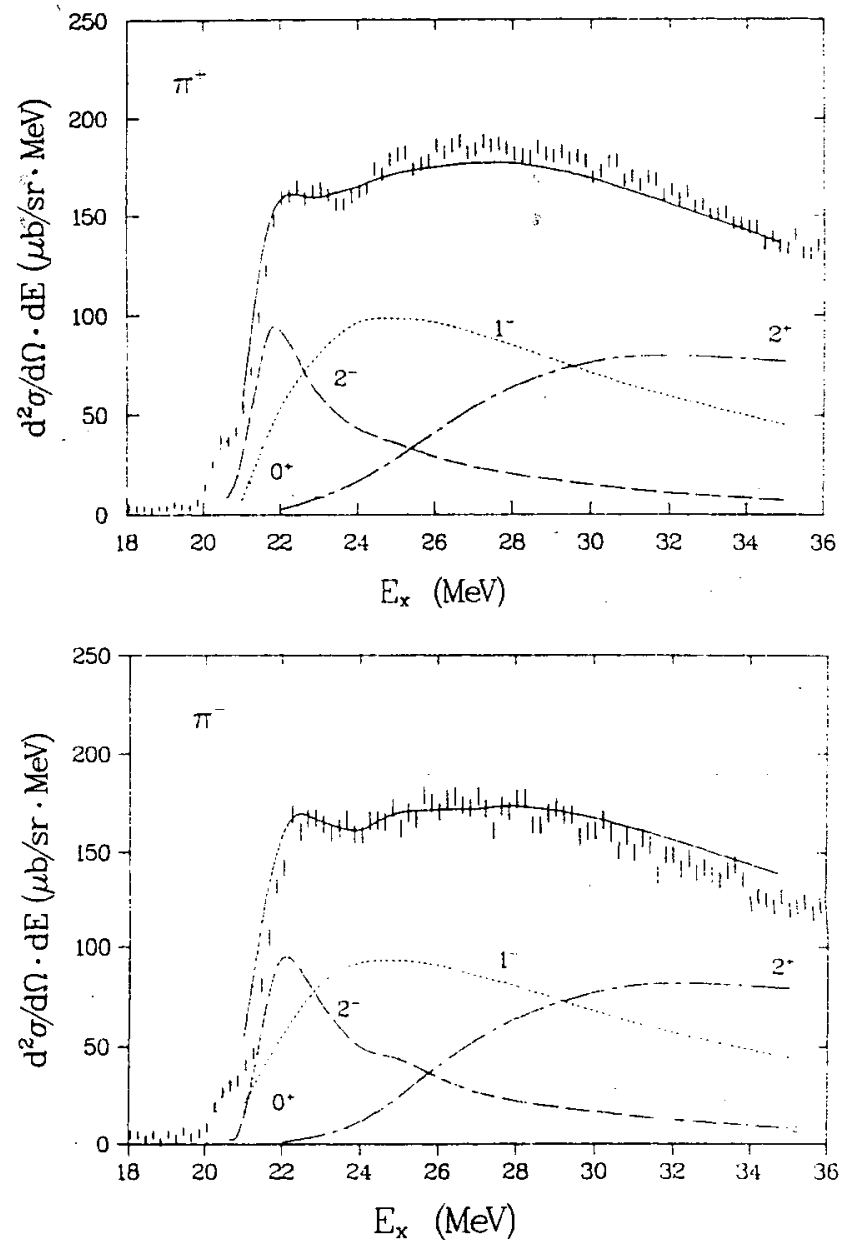

FIG. 1. Spectra of ${ }^{4} \mathrm{He}\left(\pi^{+}, \pi^{+^{\prime}}\right)$ and ${ }^{4} \mathrm{He}\left(\pi^{-}, \pi^{-\prime}\right)$ at $\theta_{\mathrm{lab}}=30^{\circ}$ and $T_{\pi}=180 \mathrm{MeV}$. The curves are the results of distorted-wave impulse-approximation (DWIA) calculations as described in the text. The DWIA curves for the $2^{-}$states have been multiplied by a factor of 1.35 for both $\pi^{+}$and $\pi^{-}$ in order to fit the data better.

$E_{x}>31 \mathrm{MeV}$, the $2^{+}$continuum makes the largest contribution to the cross sections.

- Our principal result is contained in Fig. 2 which shows near unity values for the cross-section ratio $R_{\pi}=\sigma\left(\pi^{+}\right) / \sigma\left(\pi^{-}\right)$at all $E_{x}$ beyond the threshold region $\left(E_{x} \geqslant 22 \mathrm{MeV}\right)$. Only the $\left(E_{x}\right.$-dependent) statistical and background subtraction errors are shown for $R_{\pi}$ which was averaged over $400-\mathrm{keV}$ intervals. Several sources of absolute cross-section uncertainty, such as target thicknesses and spectrometer acceptance, do not contribute to the uncertainty in $R_{\pi}$. The other sources of (mostly $E_{x}$-independent) error contribute a systematic uncertainty of $\pm 8 \%$. Averaged over the region of the $1^{-}$states $\left(E_{x}=23\right.$ to $\left.30 \mathrm{MeV}\right)$, where $R_{\gamma} \simeq 1.7 \pm 0.2$, we find $R_{\pi}=1.05 \pm 0.08$ at $\theta_{\text {lab }}=30^{\circ}$. The error of \pm 0.08 is dominated by the systematic uncertainty in $R_{\pi}$. Values of $R_{\pi}$ near 


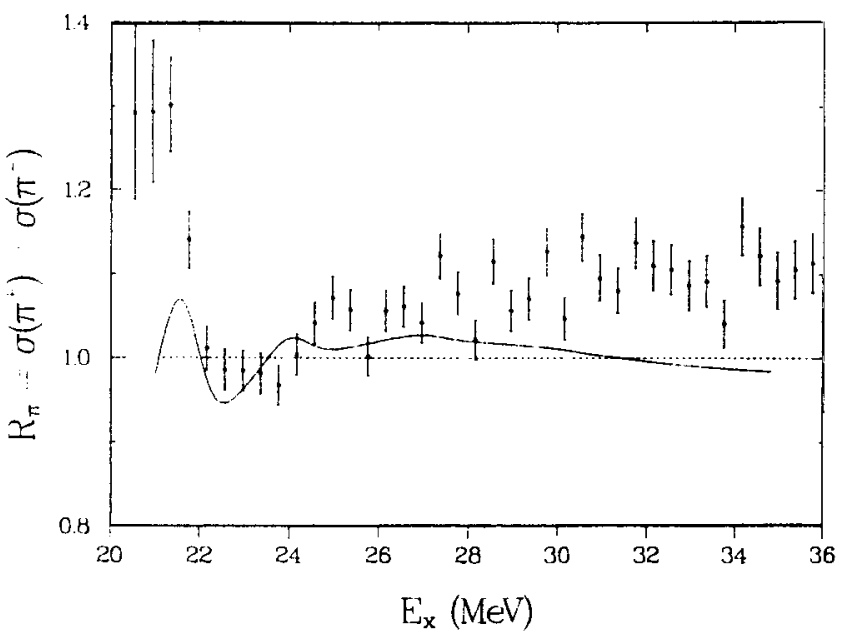

FIG. 2. $R_{\pi}$ as a function of excitation energy $E_{x}$ at $\theta_{\mathrm{lab}}=30^{\circ}$ and $T_{\pi}=180 \mathrm{MeV}$. Solid line: DWIA prediction.

threshold are larger than 1.0 because the separation threshold for protons $(19.82 \mathrm{MeV})$ is lower than that for neutrons $(20.58 \mathrm{MeV})$. At $20^{\circ}$ and $40^{\circ}$ the values of $R_{\pi}$ in the region of the $1^{-}$states are also consistent with 1.0. At $20^{\circ}$ the background was larger than at the other angles which made a realistic estimate of the error too difficult. At $40^{\circ}$ the $2^{-}$states are more strongly excited than at $30^{\circ}$ where the differential cross sections for the $1^{-}$states are expected to peak. Thus the data at $30^{\circ}$ provide the most precise determination of $R_{\pi}$ in the region of the $1^{-}$states.

In Ref. 1 the large value of $R_{\gamma}$ was attributed to isospin mixing between $T=0$ and $T=1, J^{\pi}=1^{--}$ states. Application of the two-state mixing formalism of Ref. 8 led to a ratio of the $T=0$ reaction amplitude $\left(a_{0}\right)$ to the $T=1$ amplitude $\left(a_{1}\right)$ of $\left|a_{0} / a_{1}\right|=0.13$ for the transition to an isospin-mixed state near $25 \mathrm{MeV}$, which was presumed to be predominantly a $T=1$ state. Within the two-level mixing model this value of $\left|a_{0} / a_{1}\right|$ represents a $T=0$ admixture in the $T=1$ state of amplitude $|\beta|=0.13$. Such a large admixture should be easily detectable in inelastic pion scattering because of the isospin dependence of the pion-nucleon interaction.

At pion energies near the $[3,3]$ resonance the pionnucleon interaction enhances the $T=0$ parts of the transition density amplitudes over the $T=1$ parts by a factor of +2 for $\pi^{-}$and a factor of -2 for $\pi^{+}$. Thus in the plane-wave Born approximation the crosssection ratio would be $R_{\pi}=[(\alpha-2 \beta) /(\alpha+2 \beta)]^{2}$ $=2.9$ for a $T=1$ state with a $T=0$ admixture of amplitude $\beta=-0.13$, where $\alpha=\left(1-\beta^{2}\right)^{1 / 2}$. The observed value of $R_{\pi}$ shows that isospin mixing is much smaller, i.e., $\beta$ is of the order of $1 \%$ if the two-level mixing formalism describes this case.

As pointed out in Refs. 1 and 9 , the $T=0, S=0$, $1^{-}$state in a $1 \hbar \omega$ model space is entirely due to spurious center-of-mass motion. Shell-model calculations generate higher-order nonspurious $T=0, S=0$ states but mixing with the $T=1$ states is much more complicated than in two-level ${ }^{8,9}$ mixing of isolated states. Since the ${ }^{4} \mathrm{He}$ states are all very broad and overlap, isospin mixing changes continuously with $E_{x}$ as do the contributions from $S=1$ states. For equal spectroscopic amplitudes the $S=1$ contributions to the cross sections are smaller than the $S=0$ contributions because of the relatively weaker $\pi$-nucleon spin-orbit interaction. However, they are not negligible as for photonucleon reactions.

The conclusion of small isospin mixing in ${ }^{4} \mathrm{He}$ is fully supported by distorted-wave calculations using the wave functions from the recoil-corrected continuum shell model (RCCSM). ${ }^{18}$ The Coulomb force is the only CSB force applied in the RCCSM calculations and the resulting wave functions provide very good agreement with low-energy nucleon scattering data. These include differential cross sections $(d \sigma / d \Omega)$ and $A$ for elastic scattering of neutrons from ${ }^{3} \mathrm{He}$ and protons from ${ }^{3} \mathrm{H}$, as well as $d \sigma / d \Omega, A$, and $P$ for the reaction ${ }^{3} \mathrm{H}(p, n){ }^{3} \mathrm{He}{ }^{12,13}$ They also reproduce the ${ }^{4} \mathrm{He}(\gamma, p)$ absolute cross section and the shapes of the angular distributions for the capture reactions ${ }^{3} \mathrm{He}(n, \gamma)$ and ${ }^{3} \mathrm{H}(p, \gamma) .{ }^{19-21}$ However, they predict $R_{\gamma}=1.0$. We note that, by imposing continuum boundary conditions on the excited nucleons, the RCCSM reaction calculations include the quasielastic process as well as the scattering through resonance states.

The procedure followed to calculate the pion differential cross sections $d^{2} \sigma / d \Omega d E_{x}$ by the distortedwave impulse approximation has been discussed by Halderson et al. ${ }^{22}$ The transition densities were calculated with a modified version of ALLWORLD ${ }^{23}$ and entered into the program MSUDWPI ${ }^{24}$ to generate the $d^{2} \sigma / d \Omega d E_{x}$ for $E_{x}$ between 20 and $35 \mathrm{MeV}$. For the distorted waves we employed the wave equation of Stricker, McManus, and Carr. ${ }^{25}$ The optical potential parameters were determined from the free $\pi$-nucleon phase shifts evaluated $20 \mathrm{MeV}$ below the incident pion energy in the spirit of Cottingame and Holtkamp. ${ }^{26}$ The nuclear density was of Gaussian form, $\exp \left(-r^{2} / a^{2}\right)$, with $a=1.3 \mathrm{fm}$. The DWIA cross sections were found to be quite sensitive to the distorting potentials. However, the predicted cross-section ratio $R_{\pi}$ above $23 \mathrm{MeV}$ is always near 1.0 , ranging from about 1.0 to 1.1 .

The theoretical predictions for the cross sections shown in Fig. 1 were obtained with an energydependent optical potential in the outgoing channel. They are presented for the individual multipolarities $\left(0^{+}, 2^{-}, 1^{-}, 2^{+}\right)$as well as for the sum (solid line). The $\pi^{+}$and $\pi^{-}$predictions for the $2^{-}$states were renormalized by a factor of 1.35 as suggested by the data. 
For the other states the curves shown are the predicted cross sections without a renormalization.

In conclusion, cross sections measured for inelastic pion scattering from ${ }^{4} \mathrm{He}$ show only small $\pi^{+} / \pi^{-}$ asymmetries. The absolute differential cross sections are reproduced very well by predictions using the RCCSM wave functions. The RCCSM has been very succesșful in fitting a large body of data for the mass-4 system. The only data for which the RCCSM seems to fail are the absolute cross sections for ${ }^{4} \mathrm{He}(\gamma, n)$ and thus the ratio $R_{y}$ below $E_{x}=35 \mathrm{MeV}$. Even without as thorough a theoretical treatment as the RCCSM provides, the pion scattering casts considerable doubt on the interpretation of the experimental value of $R_{\gamma}$ in terms of isospin mixing due to a large CSB force. Our data show only a very small amount of isospin mixing between the excited states of ${ }^{4} \mathrm{He}$.

The authors are indebted to Dr. R. L. Boudrie, Dr. P. J. Ellis, Dr. M. A. Franey, and Dr. E. R. Siciliano for helpful discussions and to Mr. M. K. Jones for his help with the figures. This work was supported in part by the U.S. Department of Energy, the Japan Society for Promotion of Science, the U.S.-Japan Cooperative Science Program of the National Science Foundation, and the Robert A. Welch Foundation.

(a) Present address: Los Alamos National Laboratory, Los Alamos, NM 87545.

IJ. R. Calarco, B. L. Berman, and T. W. Donnelly, Phys. Rev. C 27, 1866 (1983).

2B. L. Berman, D. D. Paul, P. Meyer, and D. L. Olson, Phys. Rev. C 22, 2273 (1980), and references therein.

3 J. R. Calarco, S. S. Hanna, C. C. Chang, E. M. Diener, E. Kuhlmann, and G. A. Fischer, Phys. Rev. C 28, 483 (1983)

${ }^{4}$ L. Ward, D. R. Tilley, D. M. Skopik, N. R. Roberson, and H. R. Weller, Phys. Rev. C 24, 317 (1981).

${ }^{5}$ D. Halderson and R. J. Philpott, Nucl. Phys. A359, 365 (1981).

${ }^{6}$ A. H. Chung, R. G. Johnson, and T. W. Donnelly, Nucl. Phys. A235, 1 (1974).

7J. T. Londergan and C. M. Shakin, Phys. Rev. Lett. 28, 1729 (1972).

${ }^{8}$ F. C. Barker and A. K. Mann, Philos. Mag. 2, 5 (1957).

${ }^{9}$ F. C. Barker, Aust. J. Phys. 37, 583 (1984).

10R. J. Philpott and D. Halderson, in The $(p, n)$ Reaction and the Nucleon-Nucleon Force, edited by C. D. Goodman et al. (Plenum, New York, 1980), p. 491

11D. Halderson and R. J. Philpott, Phys. Rev. C 28, 1000 (1983)

12T. R. Donogue et al., Phys. Rev. Lett. 37, 981 (1976).

${ }^{13 W}$. Tornow, R. C. Byrd, P. W. Lisowski, and R. L. Wolter, Nucl. Phys. A371, 235 (1981).

${ }^{14}$ C. L. Morris et al., Phys. Lett. 86B, 31 (1979), and Phys. Lett. 99B, 387 (1981).

15D. B. Holtkamp et al., Phys. Rev. Lett. 45, 420 (1980).

${ }^{16} \mathrm{H}$. A. Thiessen and S. Sobottka, Los Alamos Scientific Laboratory Report No. LA-4534-MS (unpublished).

${ }^{17}$ G. Rowe, M. Salomon, and R. Landau, Phys. Rev. C 18. 584 (1978)

${ }^{18}$ D. Halderson and R. J. Philpott, Nucl. Phys. A321, 295 (1979)

${ }^{19} \mathrm{G}$. King, K. Wienhard, J. R. Calarco, and S. S. Hanna, Stanford University Report No. 7, 1976-1977 (unpublished).

${ }^{20}$ R. C. McBroom, Ph.D. thesis, University of Florida, 1980 (unpublished).

${ }^{21}$ H. R. Weller, N. R. Roberson, G. Miter, L. Ward, and D. R. Tilley, Phys. Rev. C 25, 2111 (1982).

22D. Halderson, R. J. Philpott, J. A. Carr, and F. Petrovich, Phys. Rev. C 24, 1095 (1981).

23J. A. Carr, F. Petrovich, and J. Kelley, program ALLWORLD (unpublished).

24J. A. Carr, MSUDWPI, modified version of program DWPI of R. A. Eisenstein and G. A. Miller (unpublished).

${ }^{25} \mathrm{~K}$. Stricker, H. McManus, and J. A. Carr, Phys. Rev. C 22, 2043 (1980).

26 W. B. Cottingame and D. B. Holtkamp, Phys. Rev. Lett. 45, 1828 (1980). 


\title{
Test of the $\Delta$-Hole Model through the Energy Dependence of $\left(\pi, \pi^{\prime} \gamma\right)$ Angular Correlations on ${ }^{12} \mathrm{C}$
}

\author{
R. Olszewski, ${ }^{(a)}$ R. Baran, A. Hofmann, S. Krell, ${ }^{(b)}$ H. W. Ortner, J. Orzechowski, G. Schmidtlein, \\ and $F$. Vogler \\ Physikalisches Institut der Universität Erlangen-Nürnberg, D-8520 Erlangen, West Germany \\ G. R. Smith ${ }^{(\mathfrak{c})}$ and W. List \\ Kernforschungszentrum, Institut für Kernphysik und Institut für Experimentelle Kernphyisk \\ der Universität Karlsruhe "D-7500 Karlsruhe, West Germany \\ D. Dehnhard \\ University of Minnesota, Minneapolis, Minnesota 55455 \\ and \\ M. Thies \\ Natuurkundig Laboratorium der Vrije Universteit, 1007 MC Amsterdam. The Netherlands
}

(Received 5 August 1986)

\begin{abstract}
For the reaction ${ }^{12} \mathrm{C}\left(\pi, \pi^{\prime}\right){ }^{12} \mathrm{C}^{*}\left(2^{+}, 4.44 \mathrm{MeV}\right),{ }^{12} \mathrm{C}^{*} \rightarrow{ }^{12} \mathrm{C}$ (g.s. $)+\gamma$, pion- $\gamma$ angular correlations have been measured in and out of the reaction plane. The data taken at a pion momentum transfer of $q=0.47 \mathrm{fm}^{-1}$ for incident kinetic energies of $116,140,162,180$, and $226 \mathrm{MeV}$ confirm $\Delta$-hole model predictions, in which the $\Delta$ propagation in the nucleus is explicitly taken into account.
\end{abstract}

PACS numbers: $25.80 . \mathrm{Fm}, 14.20 . \mathrm{Gk}, 27.20 .+\mathrm{n}$

One of the most challenging goals of modern nuclear physics is to understand the role of excited nucleons in a nucleus. In this context the $\Delta(3,3)$ resonance as the lowest excited state of the nucleon with strangeness zero is of special interest. Its behavior in the nuclear medium can be studied most suitably by pion-nucleus scattering in the region of incident energies between about 100 and $300 \mathrm{MeV}$. Theoretically these medium effects are adequately considered in the so-called isobar-doorway models by the explicit introduction of isobar-hole intermediate states and an elaborate treatment of their propagation. ${ }^{1-4}$ The $\Delta$-hole model derived from this concept ${ }^{5,6}$ together with calculations in the static limit are the theoretical basis for the discussion of the data presented here.

In the $\Delta$-hole calculations starting from the opticalmodel expansion of the multiple-scattering formalism, medium corrections are taken into account by treating some properties of the $\Delta$ resonance which are different from those of the free $\Delta$ : the reduced phase space available to the nucleon in the decay of the $\Delta$ in the presence of other nucleons (Pauli blocking), binding effects, and true pion absorption in nonmesonic channels $(\Delta N \rightarrow N N)$. In the static limit the same calculations are performed with neglect of these medium corrections and the $\Delta$ propagation. ${ }^{6}$ Their results can be considered as a reference point for the dynamical phenomena to be investigated.

In inelastic pion scattering reactions to discrete final states of a nucleus the $\Delta$-hole model often yields a description of the differential cross sections nearly equivalent to that from calculations in the static limit. For these reactions the consideration of the magneticsubstate population of the excited state is of particular interest. On the one hand the transition amplitudes for the different magnetic substates are expected to be more sensitive to medium effects than the more inclusive differential cross section; on the other hand, symmetry considerations within the $\Delta$-hole model show that different excitation mechanisms populate the individual substates differently. Therefore, observables sensitive to the magnetic-substate populations are particularly suited to test the validity of the $\Delta$-hole model.

Pion-gamma angular-correlation data are sensitive to the magnetic-substate population of pion scattering to a discrete final state with subsequent gamma decay. A first $\left(\pi, \pi^{\prime} \gamma\right)$ experiment in the $\Delta$-resonance region has been performed at the Swiss Institute of Nuclear Research (SIN) at an incident pion energy of $162 \mathrm{MeV}$ on the $2^{+}(4.44 \mathrm{MeV})$ state in ${ }^{12} \mathrm{C}^{7}$ In this experiment no energy resolution was available for the scattered pions, and the angular-correlation data were taken only in the reaction plane at a single incident energy. Already from these rather modest data there were indications that the $\Delta$-nucleus dynamics was important for the description of the pion-nucleus interaction.

In this Letter we report on a new $\left(\pi, \pi^{\prime} \gamma\right)$ angular correlation experiment with considerably improved accuracy and range. The angular correlations were mea- 
sured in and out of the reaction plane at different incident energies covering the region of the $\Delta(3,3)$ resonance. The experiment was performed in the $\pi \mathrm{Ml}$ area at SIN. The nuclear state studied was again the $2^{+}(4.44 \mathrm{MeV})$ state in ${ }^{12} \mathrm{C}$. The positive pions scattered from a graphite target with an area density of about $1.70 \mathrm{~g} / \mathrm{cm}^{2}$ were detected with the SUSI spectrometer facility ${ }^{8}$ in coincidence with the deexcitation gamma rays of ${ }^{12} \mathrm{C}$. The gamma rays were detected by six movable $\mathrm{NaI}$ detectors, three of them placed in the reaction plane and three of them at $45^{\circ}$ below the reaction plane. The data discussed here were taken at a pion momentum transfer of $q=0.47 \mathrm{fm}^{-1}$ for incident kinetic energies of $116,140,162,180$, and $226 \mathrm{MeV}$. To demonstrate the quality of our data, Fig. 1 shows a spectrum of the scattered pions coincident with the 4.44-MeV deexcitation gamma rays of ${ }^{12} \mathrm{C}$ compared to the spectrum without the coincidence requirement, which is totally dominated by the peak of elastically
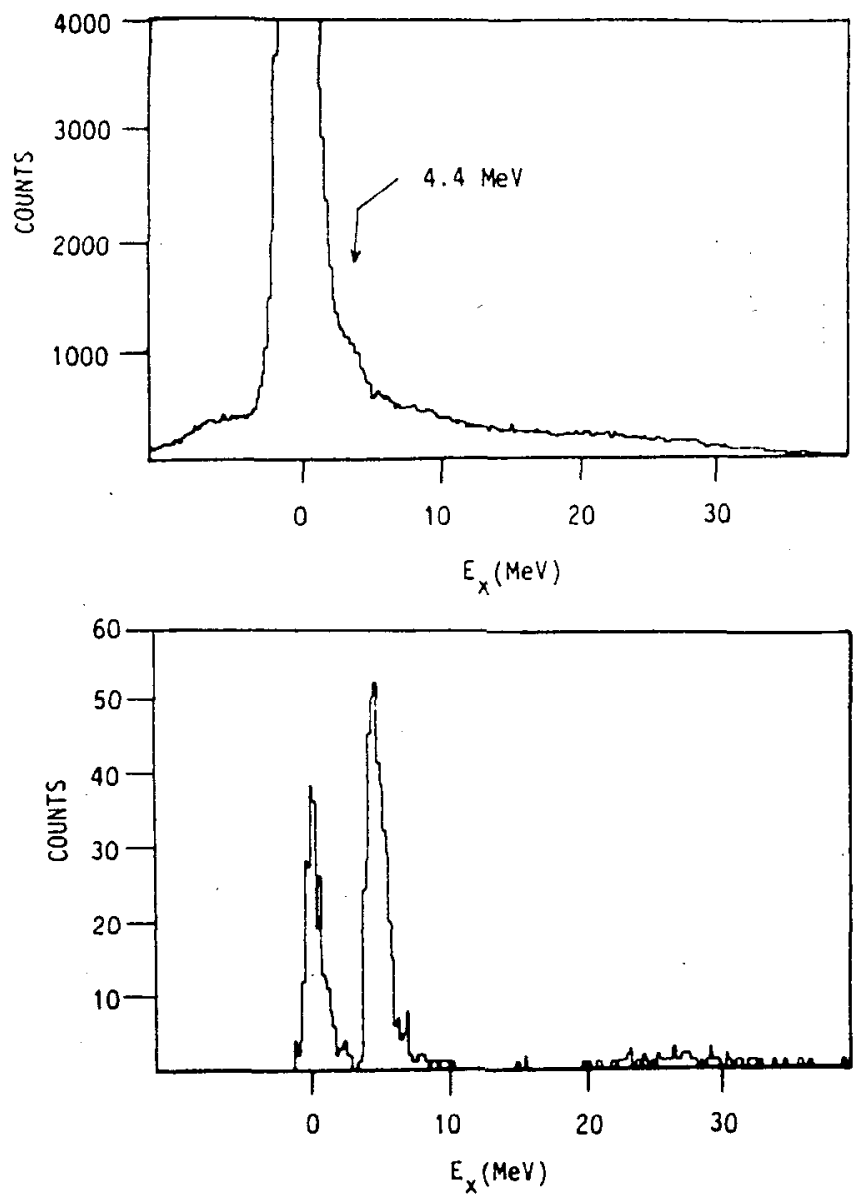

FIG. 1. Spectrum of pions with an incident kinetic energy of $162 \mathrm{MeV}$ scattered from ${ }^{12} \mathrm{C}$ at a momentum transfer of $q=0.47 . \mathrm{fm}^{-1}$. Upper part: without coincidence requirement. Lower part: in coincidence with the 4.44-MeV gamma rays detected in one of the $\mathrm{Nal}$ detectors. scattered pions. Although, as a result of random coincidences, the peak of elastically scattered pions is not completely suppressed in the coincident spectra, the inelastic events obtained from these spectra are practically free of background. A detailed description of the experimental setup together with the appropriate test procedures and monitoring systems, data analysis, and discussion of possible systematic errors will be pub-lished in a forthcoming paper. ${ }^{9}$

To present our data we use the angular-correlation function defined as

$$
W=4 \pi\left(d^{2} \sigma / d \Omega_{\pi^{\prime}} d \Omega_{\gamma}\right)\left(d \sigma / d \Omega_{\pi^{\prime}}\right)^{-1} .
$$

The determination of the differential cross section $d \sigma / d \Omega_{\pi}$, requires the determination of the yield $N_{\text {in }}$ of inelastically scattered pions. ${ }^{9}$. For the doubledifferential cross section $d^{2} \sigma / d \Omega_{\pi}, d \Omega_{\gamma}$ the yield $N_{\text {coi }}$ of coincident events has to be measured. If both yields are measured simultaneously, the angularcorrelation function $W$ can be derived from the simple relation

$$
W=4 \pi N_{\text {coi }} / N_{\text {in }} \epsilon_{\gamma} d \Omega_{\gamma},
$$

where experimental uncertainties associated with the pion spectrometer as well as the dependence on target thickness and pion flux cancel out. The remaining product $\epsilon_{\gamma} d \Omega_{\gamma}$ of efficiency and solid angle of the $\mathrm{NaI}$ detectors has been determined in a separate experiment. ${ }^{10}$ This new approach of determining the angular-correlation function $W$ itself, rather than the double-differential cross section $d^{2} \sigma / d \Omega_{\pi^{\prime}} d \Omega_{\gamma}$, as has been done so $\mathrm{far}^{7,11}$ results in the considerably improved accuracy of the data.

Choosing the quantization axis perpendicular to the reaction plane and regarding the reaction as a $0^{+} \rightarrow 2^{+} \rightarrow 0^{+}$transition, we can parametrize the angular-correlation function $W_{\text {in }}$ for measurements in the reaction plane as

$$
\begin{aligned}
W_{\text {in }}\left(\theta_{\gamma}\right. & \left.=90^{\circ}, \phi_{\gamma}\right) \\
& =A+C \sin ^{2} 2\left(\phi_{\gamma}-\phi_{2}\right) .
\end{aligned}
$$

For the out-of-plane geometry the angular correlation function $W_{\text {out }}$ is then given by

$$
\begin{aligned}
& W_{\text {out }}\left(\theta_{\gamma}=45^{\circ}, \phi_{\gamma}\right) \\
& \quad=A^{\prime}+C^{\prime} \sin ^{2} 2\left(\phi_{\gamma}-\phi_{2}\right)+D \sin ^{2}\left(\phi_{\gamma}-\phi_{0}\right),
\end{aligned}
$$

with $A^{\prime}=\frac{1}{8}(15-6 A-4 C-4 D)$ and $C^{\prime}=\frac{1}{4} C$.

The five independent parameters $A, C, D_{2} \phi_{2}$, and $\phi_{0}$ can be determined from the present experiment. They are functions of the transition amplitudes to the different magnetic substates with $m=-2,0,+2$ of the $2^{+}(4.44 \mathrm{MeV})$ state in ${ }^{12} \mathrm{C}$. The amplitudes for $m= \pm 1$ are zero according to Bohr's theorem. ${ }^{12}$ The angular-correlation functions were calculated in the 
framework of the $\Delta$-hole model and the static limit, with use of exactly the same methods and input parameters as described in Ref. 6. These calculations exhibit characteristic differences between the two approaches, particularly in their energy dependence in the region of the first minimum of the differential cross section, which corresponds to the momentum transfer of $q=0.47 \mathrm{fm}^{-1}$ chosen in our experiment. These characteristic differences, which clearly influence the pattern of the angular-correlation function, motivated us to study the energy dependence of the angular-correlation functions.

Our results are shown in Fig. 2. The solid curves are
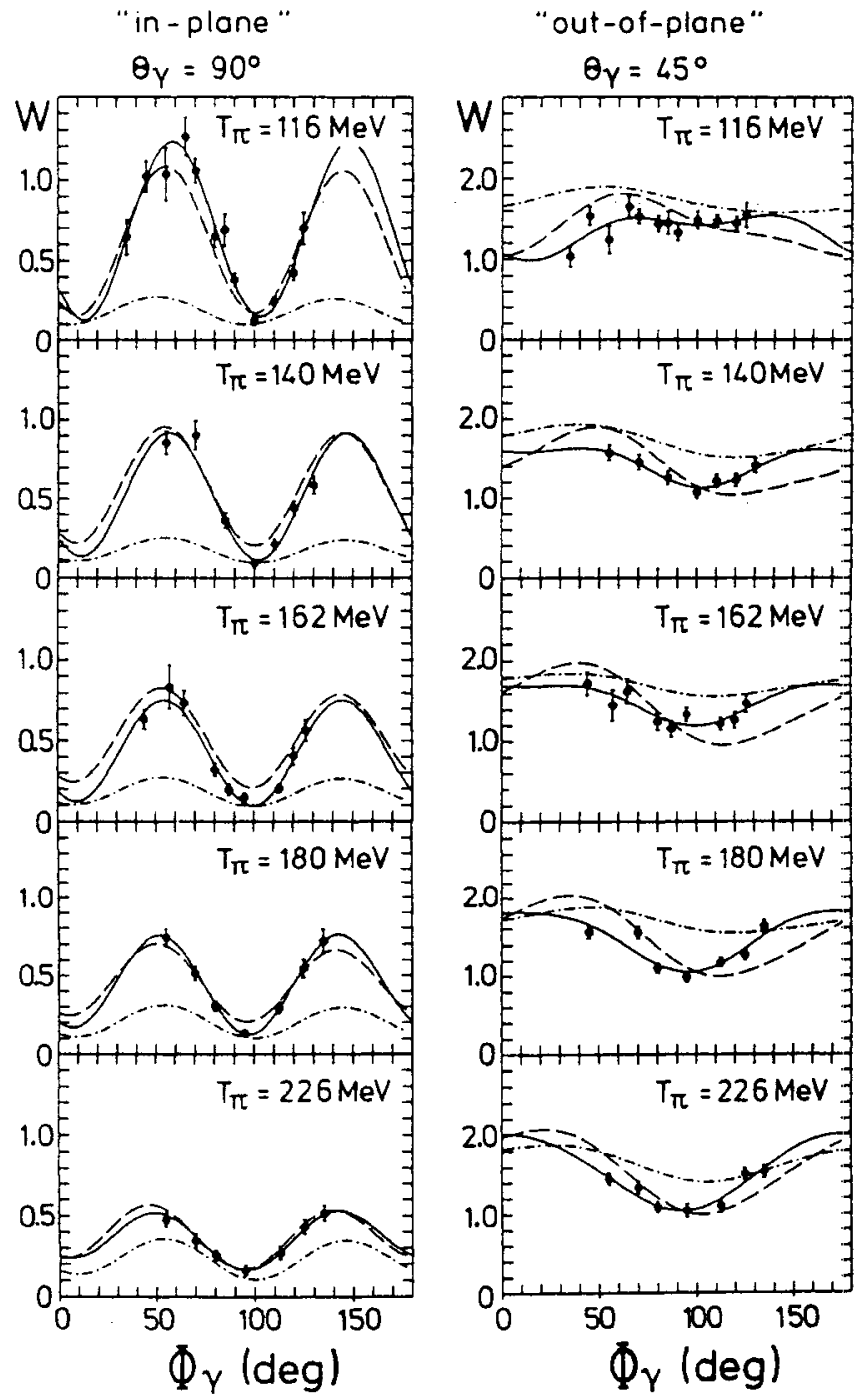

FIG. 2. In-plane and out-of-plane angular-correlation function $W$ for the reaction ${ }^{12} \mathrm{C}\left(\pi, \pi^{\prime} \gamma\right)$ at a momentum transfer of $q=0.47 \mathrm{fm}^{-1}$ for different incident kinetic energies. The solid curves are best fits according to Eqs. (3) and (4). The dashed and dot-dashed curves represent predictions of the $\Delta$-hole model and the static limit, respectively. best fits to the data points according to Eqs. (3) and (4). Their good agreement with the experimental points confirms the reliability of the present data. The dashed and dot-dashed curves are the predictions of the $\Delta$-hole model and the static limit, respectively. The finite solid angles of the detectors are taken into account by a suitable averaging of the calculations. ${ }^{9}$ Obviously, in the $\Delta$-hole model the amplitude of $W_{\text {in }}$ decreases significantly with increasing energy, whereas the amplitude of $W_{\text {out }}$ remains nearly constant. The correlation-function amplitudes in the static limit are consistently smaller and nearly energy independent. The experimental data follow the predictions of the $\Delta$-hole model. The difference between the two models depends sensitively on the nonlocality of the transition operator, expected to play an important role at small $q$, and on the distortion effects. ${ }^{6}$ We find that both of these effects are about equally important for understanding the discrepancies between the $\Delta$-hole and the static calculations, shown in Fig. 2. In any case, our data show that medium effects become more important in the region below resonance. To get more insight about this fact we plan to measure pion-gamma angular correlations as a function of the pion scattering angle.

In conclusion, we have shown that the $\Delta$-nucleus dynamics as included in the $\Delta$-hole model is needed to describe the presented $\left(\pi, \pi^{\prime} \gamma\right)$ angular-correlation data, especially in their energy dependence. Calculations in a static limit ignoring the propagation of the intermediate $\Delta$ resonance cannot reproduce the experimental data. There is, however, a convincingly good agreement with the predictions of the $\Delta$-hole model.

One of us (D.D.) is greatly indebted to the kind hospitality extended to him at SIN, where part of the work was completed. This work has been supported by the German Bundesministerium für Forschung und Technologie.

(a) Present address: Physics Department, University of British Columbia, Vancouver, B.C. V6T 2A6, Canada.

(b) Present address: Physikalisches Institut der Universität Tübingen, D-7000 Tübingen, West Germany.

(c) Present address: TRIUMF, 4004 Wesbrook Mall, Vancouver, B.C. V6T 2A6, Canada.

${ }^{1}$ L. S. Kisslinger and W. L. Wang, Ann. Phys. (N.Y.) 99, 374 (1976).

${ }^{2}$ M. Hirata, F. Lenz, and K. Yazaki, Ann. Phys. (N.Y.) 108, 116 (1977).

${ }^{3} \mathrm{~K}$. Klingenbeck, M. Dillig, and M. G. Huber, Phys. Rev. Lett. 41, 387 (1978).

${ }^{4}$ E. Oset and W. Weise, Nucl. Phys. A329, 365 (1979).

${ }^{5}$ M. Hirata, J. H. Koch, F. Lenz, and E. J. Moniz, Ann. Phys. (N.Y.) 120, 205 (1979); Y. Horikawa, M. Thies, and F. Lenz, Nucl. Phys. A345, 386 (1980). 
${ }^{6} \mathrm{~F}$. Lenz, M. Thies, and Y. Horikawa, Ann. Phys. (N.Y.) 140, 266 (1982).

${ }^{7}$ F. Vogler, R. Olszewski, M. Meyer, E. L. Mathie, G. R. Smith, E. Boschitz, S. Chakravarti, D. Dehnhard, and M. Thies, Phys. Lett. B 134, 161 (1984).

8J. P. Albanese, J. Arvieux, E. T. Boschitz, R. Corfu, J. P. Egger, P. Gretillat, C. H. Q. Ingram, C. Lunke, E. Pedroni, C. Perrin, J. Piffaretti, L. Pflug, E. Schwartz, C. Wiedner, and J. Zichy, Nucl. Instrum. Methods 158, 363 (1979).

${ }^{9} \mathrm{R}$. Olszewski et al., to be published.

10S. Krell, A. Hofmann, R. Olszewski, H. W. Ortner, and F. Vogler, to be published.

IIR. J. Sobie, T. E. Drake, J. Gaydos, R. R. Johnson, R. Tacik, D. R. Gill, B. K. Jennings, and N. de Takacy, Phys. Lett. B143, 338 (1984).

12 A. Bohr, Nucl. Phys. 10, 486 (1959). 


\title{
${ }^{12} \mathbf{C}\left(\pi^{ \pm}, \pi^{ \pm \prime} p\right)^{1 /} B$ Reaction near the Giant Dipole Region
}

\author{
Sung Hoon Yoo, A. Williams, S. Mordechai, (a) and C. Fred Moore \\ Unicersity of Texas at Austin. Austin. Texas 78712-1081 \\ C. L. Morris and S. J. Seestrom-Morris : \\ Los Alamos National Laboratory, Los Alamos, New Mexico 87545
}

M. K. Jones, S. M. Sterbenz, and D. Dehnhard

University of Minnesota. Minneapolis. Minnesota 55453

D. S. Oakley

Unicersity of Colorado, Boulder. Colorado 80309

A. Fazely

Louisiana State University, Baton Rouge, Louisiana 70803

(Received 13 February 1989)

\begin{abstract}
Cross sections for the ${ }^{12} \mathrm{C}\left(\pi^{ \pm}, \pi^{ \pm \prime} p\right){ }^{\prime \prime} \mathrm{B}$ reaction have been measured and compared with the results of distorted-wave impulse-approximation calculations that employ the factorization approximation for quasifree scattering. Near the giant dipole resonance of ${ }^{12} \mathrm{C}$ the calculations underestimate the cross sections for $\left(\pi^{-}, \pi^{-} p\right)$. The angular distributions for $\left(\pi^{+}, \pi^{+\prime} p\right)$ and $\left(\pi^{-}, \pi^{-\prime} p\right)$ are different and indiwate that in this region there is interference between direct decay and semidirect decay.
\end{abstract}

P.ACS numbers: $25.80 . F m, 24.30 . C \% 27.20 .+n$

The response of the continuum of nuclear states above nucleon breakup threshold to inelastic scattering has been the subject of intensive research. ${ }^{1}$ Transitions to the giant resonances (GR's) can proceed in competition with the quasielastic scattering process. Thus, a detailed understanding of the reaction mechanism is needed in order to unravel the structure of the continuum. For example, the extent to which isospin is a good quantum number for the giant resonances is of great importance in the siudy of nuclear structure. ${ }^{2}$ We believe that coincidence measurements between inelastically scattered probes and emitted secondary particles provide a powerful tool for these investigations. The isospin selectivity of $\pi^{+}$and $\pi^{-}$scattering makes pion probes particularly useful in a study of the continuum.

Coincidence experiments involving pions as a probe can be divided into two groups: experiments in the region where quasielastic scattering dominates [ $E_{x}$ (target) $\geq 40 \mathrm{MeV}$ and backward angles] ${ }^{3-7}$ and experiments in the region of the giant resonances $\left[E_{x}\right.$ (target) $\leq 4.0$ $\mathrm{MeV}$ and forward angles]. ${ }^{8.9}$ Indeed, Chant, Rees, and Roos 11 have shown that quasifree single-nucleon knockout calculations, carried out with the code TIIRIELILL: ${ }^{11}$ are in good agreement with the measured ${ }^{2} \mathrm{C}\left(\pi^{+}, \pi^{+\prime} p\right)^{\prime \prime} \mathrm{B}$ (g.s.) cross sections of Ziock et al. ${ }^{4}$ at large momentum transfer and high $E_{x}\left({ }^{12} \mathrm{C}\right)$. These calculations use a factorized form of the distorted-wave impulse approximation (DWIA), and include optical-model distortions of the incoming pion, outgoing pion, and final-state proton. The processes that contribute to the lower $E_{1}$ (target) of the continuum, however, are more complicated. It has been suggested ${ }^{9.12}$ that, in this GR region, direct decay (due to quasifree-knockout scattering) and semidirect decay (due to resonant inelastic scattering to states of good isospin) compete with each other in a coherent way.

The current study is aimed at the excitation region near the giant dipole resonance (GDR) of ${ }^{12} \mathrm{C}$. In this Letter, we compare cross sections for the ${ }^{12} \mathrm{C}\left(\pi^{ \pm}, \pi^{ \pm \prime} p\right)$ reaction with DWIA calculations performed with the code THREEDEE in order to evaluate the importance of quasifree-knockout scattering in the excitation-energy region near the GDR. We also present values for the ratio $R=\sigma\left(\pi^{+}, \pi^{+\prime} p\right) / \sigma\left(\pi^{-}, \pi^{-1} p\right)$, which may provide information on the isospin structure of the continuum.

The experiment was performed on the Energetic Pion Channel and Spectrometer (EPICS) ${ }^{13}$ at Los Alamos National Laboratory. Scattered pions were detected in the focal plane of the high-resolution EPICS spectrometer. Protons were detected in coincidence with the scattered pions using five plastic-BGO (bismuth-germanate) "phoswich" detectors mounted in the vacuum scattering chamber. Measurements were made at an incident pion energy of $180 \mathrm{MeV}$. The target was a carbon foil of natural isotopic composition and of areal density $91 \mathrm{mg} / \mathrm{cm}^{2}$ mounted at $60^{\circ}$ with respect to the beam direction. The energy loss in the detector entrance foil and in the target limited the minimum detectable proton energy to about $4 \mathrm{MeV}$ (at the center of the target). The pion scattering angle $\theta_{\text {litb }}$ was chosen to be $20^{\circ}$, near the maximum in the angular distribution for the GDR in "C. "Protons were detected at laboratory scattering angles $H_{: 1}$ $=-120^{\circ},-90^{\circ},-60^{\circ}, 60^{\circ}$, and $90^{\circ}$ with respect to the incident beam. A relative measure of the pion beam 
intensity was provided by a toroidal pickoff upstream of the pion production target which measured the primary proton beam current. Typical average pion fluxes were $\approx 2.8 \times 10^{7} / \mathrm{sec}$ for $\pi^{+}$and $1.5 \times 10^{7} / \mathrm{sec}$ for $\pi^{-}$. The data were normalized by comparing yields measured for $\pi^{+}$and $\pi^{-}$scattering from hydrogen (using a $\mathrm{CH}_{2}$ target of areal density $73 \mathrm{mg} / \mathrm{cm}^{2}$ ) to cross sections calculated using the $\pi-N$ phase shifts of Rowe, Salomon, and Landau. ${ }^{15}$ The energy resolution in the $\left(\pi, \pi^{\prime}\right)$ and $\left(\pi, \pi^{\prime} p\right)$ reactions is about $200 \mathrm{keV}$ and $4 \mathrm{MeV}$, respectively.

Missing-mass spectra for $\pi^{+}$and $\pi^{-}$scattering are shown in Fig. 1. Singles spectra for $\pi^{+}$(red) and $\pi^{-}$ (blue) are presented in the upper portion. The $\pi^{+}$and $\pi^{-}$cross sections are nearly equal everywhere as expected for a self-conjugate nucleus because of charge symmetry except for a known isospin-mixed doublet near 19 MeV. ${ }^{16}$

Figure 1(b) shows the pion missing-mass spectrum, gated by the requirement of detecting a coincident proton at an energy which implies that the excitation energy of the residual nucleus, ${ }^{11} \mathrm{~B}$, is less than $10 \mathrm{MeV}$, and summed over all proton detectors. In contrast to the singles spectra, the $\left(\pi, \pi^{\prime} p\right)$ coincidence yields are larger for $\pi^{+}$than $\pi^{-}$throughout the spectrum. The missingmass spectrum gated by detecting a proton leading to excited states of ${ }^{11} \mathrm{~B}$ higher than $10 \mathrm{MeV}$ is plotted in Fig.

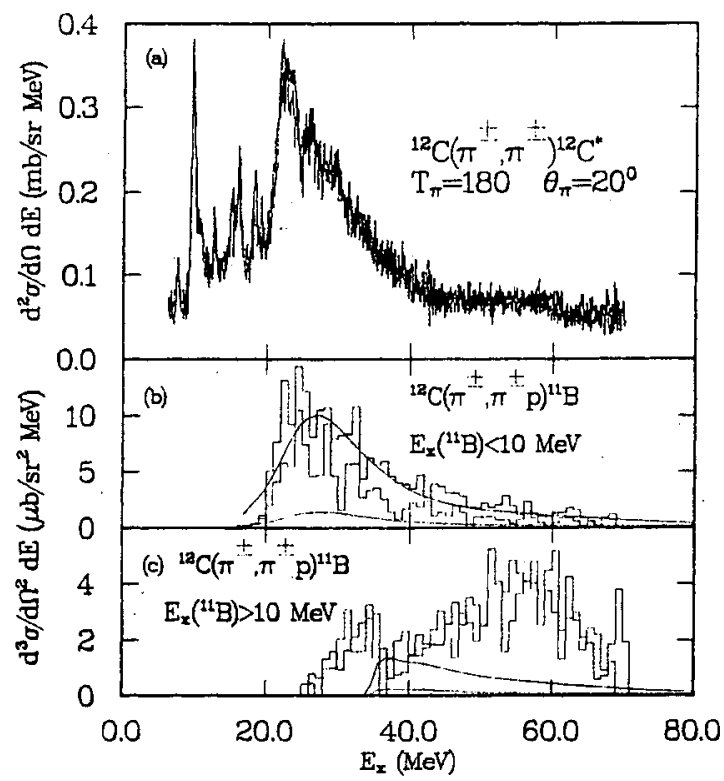

FIG. 1. Missing-mass spectra: blue for $\pi^{-}$and red for $\pi^{+}$. (a) ${ }^{12} \mathrm{C}\left(\pi^{ \pm}, \pi^{ \pm \prime}\right)^{12} \mathrm{C}^{*}$ singles spectra at $\theta_{\pi}=20^{\circ}$ and $T_{\pi}=180$ $\mathrm{MeV}$. (b) ${ }^{\prime 2} \mathrm{C}\left(\pi^{ \pm}, \pi^{ \pm} p\right)^{\prime \prime} \mathrm{B}$ coincidence spectra, gated by $E_{.}\left({ }^{\prime \prime} \mathrm{B}\right)<10 \mathrm{MeV}$. Red (blue) solid curve is a DWIA calculation of $\pi^{+}\left(\pi^{-}\right)$1p-shell knockout. (c) ${ }^{12} \mathrm{C}\left(\pi^{ \pm}, \pi^{ \pm} p\right)^{\prime \prime} \mathrm{B}$ reaction, gated by $E_{x}\left({ }^{\prime \prime} \mathrm{B}\right) \geq 10 \mathrm{MeV}$. Red (blue) solid curve is a DWIA calculation of $\pi^{+}\left(\pi^{-}\right)$is-shell knockout.
1 (c). In this case, which includes multiparticle breakup states, the $\left(\pi^{+}, \pi^{+\prime} p\right)$ and $\left(\pi^{-}, \pi^{-\prime} p\right)$ cross sections are about the same at all energies. The $4-\mathrm{MeV}$ threshold for proton detection in our phoswich detectors seems to have a minimal effect on the results of our coincidence data, since we are considering the decay of ${ }^{12} \mathrm{C}^{*}$ states above $E_{x}=20 \mathrm{MeV}$.

The inequality of the $\left(\pi^{-}, \pi^{-1} p\right)$ and $\left(\pi^{+}, \pi^{+} p\right)$ cross sections for $E_{x}\left({ }^{\prime \prime} \mathrm{B}\right)<10 \mathrm{MeV}$ can be qualitatively understood by simple arguments. If the reaction process is quasifree knockout, we expect a ratio $R$ close to 9 as for $\pi^{+}$and $\pi^{-}$scattering from free protons. Nearly as large a ratio is obtained from the factorized DWIA calculations performed with the code THREEDEE. The curves plotted in Fig. 1 (b) correspond to scattering from a proton bound in the $1 p$ shell. A spectroscopic factor $C^{2} S=3.98$, the summed $1 p$-shell spectroscopic factor predicted by Cohen and Kurath, ${ }^{17}$ and a separation energy $S_{p}=15.8 \mathrm{MeV}$ were assumed. The calculations in Fig. 1 (c) are for scattering from a $1 s$-shell proton with $C^{2} S=2.0$, the value of the shell-model limit, and $S_{f}$, $=34.3 \mathrm{MeV}$. Both sets of calculations show $\pi^{+}$scattering to be larger than $\pi^{-}$by nearly the free $\pi$-p ratio (for example, we calculate $R-7.36$ for $1 p$ knockout near the GDR). However, the experimental ratio is only 1.59 on the average (Table I).

Both the shape and magnitude of the $\left(\pi^{+}, \pi^{+} p\right)$ spectrum gated by $E_{x}\left({ }^{\prime} \mathrm{B}\right)<10 \mathrm{MeV}$ are well described by the DWIA. However, the $\left(\pi^{-}, \pi^{-\prime} p\right)$ data for the same gate are much larger than the predicted values [Fig. [(b)]. This discrepancy would be larger if we had used the $1 p$-shell spectroscopic factor of 2.9 and $1 s$-shell spectroscopic factor of 1.8 reported in Ref. 10. For the data from $E_{x}$ ("' $\left.\mathrm{B}\right)>10 \mathrm{MeV}$ gate, the agreement between experiment and the DWIA prediction is poor for both $\pi^{+}$and $\pi^{-}$scattering [Fig. 1(c)].

The angular distributions of the emitted protons are plotted in Fig. 2 as a function of the outgoing proton angle in the center-of-mass system of the recoil ${ }^{12} \mathrm{C}$. The cross section for events leading to the ground state or the low-excitation states of ${ }^{11} \mathrm{~B}$, i.e., $E_{x}\left({ }^{\prime \prime} \mathrm{B}\right)<10 \mathrm{MeV}$, summed over $E_{x}\left({ }^{12} \mathrm{C}\right)$ from 20 to $30 \mathrm{MeV}$, is shown in

TABLE 1. The ratios $R=\sigma\left(\pi^{+}, \pi^{+} p\right) / \sigma\left(\pi^{-}, \pi^{-} p\right)$ at each proton detector and the ratio of summed cross section. The $\sigma\left(\pi^{ \pm}, \pi^{ \pm \prime} p\right)$ data were obtained by summing cross sections from 20 to $30 \mathrm{MeV}$ in $E_{\mathrm{r}}\left({ }^{12} \mathrm{C}\right)$ with the $E_{x}\left({ }^{11} \mathrm{~B}\right)<10 \mathrm{MeV}$ gate, at $T_{\pi}=180 \mathrm{MeV}$ and $\theta_{\text {lat }}=20^{\circ}$.

\begin{tabular}{ccc}
\hline$\theta_{p \mathrm{c} . \mathrm{m} .}$ & $R$ (DWIA) & $R$ (Experiment) \\
\hline$-60.3^{\circ}$ & 8.59 & $1.99 \pm 0.41$ \\
$-28.5^{\circ}$ & 7.89 & $3.09 \pm 0.62$ \\
$3.9^{\circ}$ & 6.75 & $2.50 \pm 0.61$ \\
$127.3^{\circ}$ & 5.29 & $0.47 \pm 0.12$ \\
$155.5^{\circ}$ & 6.02 & $0.33 \pm 0.11$ \\
$R$ of summed $\sigma$ & 7.36 & $1.59 \pm 0.16$ \\
\hline
\end{tabular}




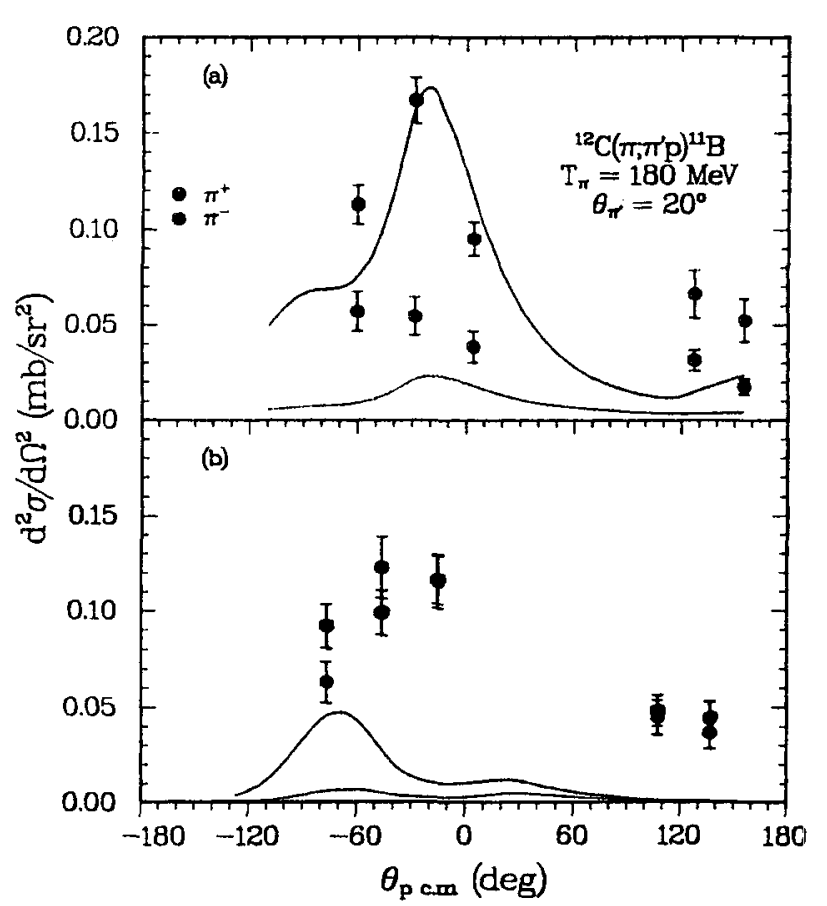

FIG. 2. Angular distribution in the center-of-mass system of the recoil ${ }^{12} \mathrm{C}^{*}$. (a) The missing-mass spectra were summed over $E_{x}\left({ }^{12} \mathrm{C}\right)=20$ to $30 \mathrm{MeV}$ and $E_{x}\left({ }^{11} \mathrm{~B}\right)<10 \mathrm{MeV}$. Red (blue) solid curve is a DWIA calculation of $\pi^{+}\left(\pi^{-}\right)$lp-shell knockout. (b) The missing-mass spectra were summed over $E_{1}\left({ }^{\prime 2} \mathrm{C}\right)=41$ to $70 \mathrm{MeV}$ and $E_{x}\left({ }^{11} \mathrm{~B}\right) \geq 10 \mathrm{MeV}$. Red (blue) solid curve is a DWIA calculation of $\pi^{+}\left(\pi^{-}\right) \mid s$-shell knockunt calculations.

Fig. 2(a). In Fig. 2(b) data for the higher-excitation states of ${ }^{11} \mathrm{~B}, E_{x}\left({ }^{\prime \prime} \mathrm{B}\right) \geq 10 \mathrm{MeV}$, were summed over $E_{x}\left({ }^{12} \mathrm{C}\right)$ from 41 to $70 \mathrm{MeV}$. The curves are the DWIA calculations described earlier. The $\left(\pi^{+}, \pi^{+} p\right)$ data with the $E_{x}\left({ }^{11} \mathrm{~B}\right)<10 \mathrm{MeV}$ gate are reasonably well described by the DWIA. However, the $\left(\pi^{-}, \pi^{-1} p\right)$ data exhibit no clear peak in the recoil direction in contradiction to the DWIA. In Table I we present the ratio. $R$ of the data leading to $E_{x}\left({ }^{11} \mathrm{~B}\right)<10 \mathrm{MeV}$ and the DWIA predictions for $1 p$-shell knockout summed from 20 to 30 $\mathrm{MeV}$ of $E_{x}\left({ }^{12} \mathrm{C}\right)$. The experimental value of $R$ varies from a maximum of $R=3.09 \pm 0.62$ near the quasifreeknockout direction to a minimum of $R=0.33 \pm 0.11$ in the opposite direction. However, the calculations give $R$ $=8$ and 6 at these two angles, respectively.

The calculations discussed so far include only the quasifree-knockout process. Another process that can contribute to this $\left(\pi^{ \pm}, \pi^{ \pm \prime} p\right)$ reaction in the GR region is a semidirect one where the pion excites a state of ${ }^{12} \mathrm{C}$ in the GR region that subsequently decays through emission of a proton. If the state in ${ }^{12} \mathrm{C}$ has good isospin and if the difference between the neutron and proton penetrabilities is neglected, $R$ must be equal to 1 at all emitted proton angles. In this case the decay of the state is governed by branching ratios and these are independent of the manner in which the state was created. Furthermore, the angular distribution should be symmetric about $90^{\circ}$. The data for $20 \leq E_{x}\left({ }^{12} \mathrm{C}\right) \leq 30 \mathrm{MeV}$ with the $E_{x}\left({ }^{11} \mathrm{~B}\right)<10 \mathrm{MeV}$ gate could be qualitatively explained by a mixture of these two processes: direct (due to quasifree knockout) and semidirect (due to inelastic scattering to states of good isospin). The angular distributions of the decay proton indicate that the $\left(\pi^{+}, \pi^{+\prime} p\right)$ is dominated by the direct decay whereas, at least, the $\left(\pi^{-}, \pi^{-i} p\right)$ must have a strong contribution from the semidirect process. This process must, of course, also contribute to $\left(\pi^{+}, \pi^{+} p\right)$, although the good agreement between absolute experimental cross sections and THREEDEE predictions suggests a predominance of the quasifree process. We propose that the observed strong angle dependence of $R$ is probably due to an interference between the amplitudes for these two processes.

In order to describe a situation which lies between the limits of quasifree knockout and the excitation and decay of states of good isospin, one may use a form of the doorway model. ${ }^{18}$ In this model, the interaction of the pion probe with the nucleus leads to proton particle-hole and neutron particle-hole states in the continuum with amplitudes approximately in the ratio of the free pion-nucleon couplings. These continuum states couple either to the GR states with width $\Gamma_{R}$, or they decay directly into a potential scattering state with width $\Gamma_{D}$ (the quasifree process). The decay of the GR states would lead to equal amplitudes for proton and neutron emission, i.e., $R=1$, but the interference with the quasifree process causes $\pi^{+} / \pi^{-}$asymmetries. The enhancement of the proton decay observed in the current $\left(\pi^{-}, \pi^{-1} p\right)$ data above the DWIA calculations indicates that $\Gamma_{R}$ and $\Gamma_{D}$ must be comparable in size for the continuum near the $G R$ region of ${ }^{12} \mathrm{C}$. This interference would be less important for $\left(\pi^{+}, \pi^{+\prime} p\right)$ because the direct amplitude is 3 times larger than for $\left(\pi^{-}, \pi^{-1} p\right)$. Therefore, the decay of the continuum in the region of the GR is largely governed by how it was excited. The absolute cross sections predicted by THREEDEE depend on the choice of spectroscopic factors, but not on the ratio $R$. Thus, we base our conclusion on the failure of simple DWIA calculations to reproduce $R$. We have made no attempt to include higher-order effects in the $\pi$-nucleus scattering such as those which have been predicted to arise from the $\Delta$-hole model. ${ }^{7}$

These observations in the region of the GDR in ${ }^{12} \mathrm{C}$ can be contrasted with the result obtained at higher excitations energies in ${ }^{12} \mathrm{C}$ [Figs. $1(\mathrm{c})$ and $2(\mathrm{~b})$ ]. In this high-excitation region, $40 \leq E_{x}\left({ }^{12} \mathrm{C}\right) \leq 70 \mathrm{MeV}$, we find that more than half of the cross section seen in the coincidence spectrum corresponds to the data leading to $E_{x}\left({ }^{\prime \prime} \mathrm{B}\right) \geq 10 \mathrm{MeV}$. For these events we observe a broad bump in Fig. 1 (c) centered near $55 \mathrm{MeV}$ of excitation in ${ }^{12} \mathrm{C}$. The angular distribution of protons associated with this bump also appears to peak near the recoil 
direction but the ratio $R$ [Fig. 2(b)] is near unity at all angles. The DWIA calculations for $1 s$-shell knockout do not resemble these data at all. The near equality of the $\left(\pi^{+}, \pi^{+\prime} p\right)$ and $\left(\pi^{-}, \pi^{-1} p\right)$ cross sections suggests that protons and neutrons are involved equally in the reaction. However, we do not believe that, for $E_{x}\left({ }^{12} \mathrm{C}\right) \geq 40$ $\mathrm{MeV}$, states of good isospin would play a major role except for possible double resonances. ${ }^{19}$ It is also possible that direct two-, three-, and four-nucleon removal are important here. Lourie et al. ${ }^{20}$ also observed considerable strength in this region of $E_{x}\left({ }^{12} \mathrm{C}\right)$ in the ${ }^{12} \mathrm{C}\left(e, e^{\prime} p\right)$ reaction. They interpreted this strength as due to multinucleon reaction mechanisms. The failure of the onenucleon-knockout calculations with THREEDEE to reproduce the magnitude and the near equality of the $\pi^{+}$and $\pi^{-}$data suggests the importance of one of these processes for $\left(\pi, \pi^{\prime} p\right)$.

In summary, we have measured cross sections for the ${ }^{12} \mathrm{C}\left(\pi^{ \pm}, \pi^{ \pm \prime} p\right)$ reactions, and have compared the results with the factorized DWIA calculations. Neither the DWIA calculations, which assume a quasifree-knockout process, nor the assumption that the reaction is dominated by states of good isospin in ${ }^{12} \mathrm{C}$ (the GDR) can explain the data. This leads to the speculation that inelastic scattering to the giant dipole region in ${ }^{12} \mathrm{C}$ contains two components, one of which is direct (quasifree), the other being semidirect (resonance). The ratio $R$ shows an angular dependence that indicates coherent interference between these amplitudes.

The authors thank Professor N. Chant and Professor Phil Roos for sending us the code THREEDEE and for useful discussions and Dr. Richard Silbar for helpful conversations. This work was supported in part by the U.S. Department of Energy, the Robert A. Welch Foundation, and the U.S.-Israel Binational Science Foundation.

\footnotetext{
(a) Permanent address: Ben-Gurion University of the Negev, Beer-Sheva 84l05, 1srael.

'C. C. Chang, in Giant Multiple Resonance, edited by F. E. Bertrand (Harwood-Academic, Chur, Switzerland, 1979), p. 191.
}

'S. S. Hanna, in Ref. 1, p. 1.

${ }^{3}$ H. J. Ziock, R. J. Ellis, K. O. H. Ziock, J. Bolger. E. Boschitz, J. Arvieux, R. Corfu, and J. Piffaretti, Phys. Rev. Lett. 43, 1919 (1979).

${ }^{4}$ H. J. Ziock, R. J. Ellis, K. O. H. Ziock, J. Bolger. E. Boschitz, J. Arvieux, R. Corfu, and J. Piffaretti, Phys. Rev. C 24, 2674 (1981).

${ }^{5}$ E. Piasetzky, D. Ashery, A. Altman, A. I. Yavin, F, W. Schlepütz, R. J. Powers, W. Bertl, L. Felawka, H. K. Walter, R. F. Winter, and J. v. d. Pluym, Phys. Rev. Lett. 46, 1271 (1981); Phys. Rev. C 25, 2687 (1982).

6J. R. Hurd, J. S. Boswell, R. C. Minehart, L. B. Rees, Y. Tzeng, H. J. Ziock, and K. O. H. Ziock, Nucl. Phys. A462, 605 (1987).

${ }^{7}$ G. S. Kyle, P.-A. Amaudruz, Th. S. Bauer, J. J. Domingo, C. H. Q. Ingram, J. Jansen, D. Renker, J. Zichy, R. Stamminger, and F. Vogler, Phys. Rev. Lett. 52, 974 (1984).

${ }^{8}$ M. Jones, Ph.D. thesis, University of Minnesota, 1989 (unpublished).

${ }^{9}$ Christopher L. Morris, Bull. Am. Phys. Soc. 33, 1093 (1988).

${ }^{10}$ N. A. Chant. L. Rees, and P. G. Roos, Phys. Rev. Lett. 48, 1784 (1982).

${ }^{\prime \prime}$ N. S. Chant, computer code THREEDEE; L. Rees, N. S. Chant, and P. G. Roos, Phys. Rev. C 26, 1580 (1982).

${ }^{12} \mathrm{H}$. Überall, Electron Scattering from. Complex Nuclei (Academic, New York, 1979), Vol. B, p. 729.

${ }^{13}$ H. A. Thiessen and S. Sobottka, Los Alamos National Laboratory Report No. LA-4534-MS (unpublished).

${ }^{14}$ L. C. Bland, Ph.D. thesis, University of Pennsylvania, 1984 (unpublished); Los Alamos National Laboratory Report No. LA-9960-T, 1984 (unpublished).

${ }^{15}$ G. Rowe, M. Salomon, and R. H. Landau, Phys. Rev. C 18. 584 (1978).

${ }^{16}$ W. B. Cottingame, K. G. Boyer, W. J. Braithwaite, S. J. Green, C. J. Harvey, R. J. Joseph, D. B. Holtkamp, C. Fred Moore, J. J. Kraushaar, R. J. Peterson, R. A. Ristinen, J. R. Shepard, G. R. Smith, R. L. Boudrie, N. S. P. King, C. L. Morris, J. Piffaretti, and H. A. Thiessen, Phys. Rev. C 36, 230 (1987).

${ }^{17}$ S. Cohen and D. Kurath, Nucl. Phys. A 101, I (1967).

${ }^{18}$ H. Feshbach, A. K. Kerman, and R. H. Lemmer, Annu. Rev. Phys. 41, 230 (1967).

${ }^{19} \mathrm{~S}$. Mordechai, N. Auerbach, M. G. Burlein, H. T. Fortune, S. J. Greene, C. Fred Moore, C. L. Morris, J. M. O'Donnell. M. W. Rawool, J. D. Silk, D. L. Watson, S. H. Yoo, and J. O. Zunbro, Phys. Rev. Lett. 61, 531 (1988).

${ }^{20} \mathrm{R}$. W. Lourie et al., Phys. Rev. Lett. 56, 2364 (1986). 


\title{
Asymmetry Measurement of Pion Elastic Scattering from Polarized ${ }^{13} \mathrm{C}$ in the Energy Region of the $\boldsymbol{P}_{33}$ Resonance
}

\author{
Yi-Fen Yen, B. Brinkmöller, ${ }^{(a)}$ D. Dehnhard, S. M. Sterbenz, and Yi-Ju Yu \\ University of Minnesota, Minneapolis, Minnesota 55455
}

Brian Berman, (b) G. R. Burleson, K. Cranston, A. Klein, (c) and G. S. Kyle

New Mexico State University, Las Cruces, New Mexico 88003

R. Alarcon, T. Averett, J. R. Comfort, J. J. Görgen, B. G. Ritchie, and J. R. Tinsley ${ }^{(d)}$

Arizona State Unicersity, Tempe, Arizona 85287

M. Barlett, G. W. Hoffmann, K. Johnson, C. F. Moore, M. Purcell, H. Ward, and A. Williams

University of Texas at Austin, Austin. Texas 78712-1081

J. A. Faucett, S. J. Greene, J. J. Jarmer, J. A. McGill, (e) C. L. Morris, S. Penttilä, and N. Tanaka

Los Alamos National Laboratory, Los Alamos. New Mexico 87545

H. T. Fortune, E. Insko, R. Ivie, J. M. O'Donnell, and D. Smith

University of Pennsylvania, Philadelphia, Pennsylvania 19104

M. A. Khandaker

University of Marylana', College Park, Maryland 20742

S. Chakravarti

California State Polytechnic University, Pomona, California 91768

(Received II December 1990)

\begin{abstract}
Analyzing powers $A_{y}$ were measured for $\pi^{+}$and $\pi^{-}$elastic scattering from polarized ${ }^{13} \mathrm{C}$ at energies near the $P_{33}$ resonance. At $T_{x}=132 \mathrm{MeV}$ the values of $A_{y}$ are significantly different from zero for $\pi^{-}$. For $\pi^{+}$at $132 \mathrm{MeV}$ and for both $\pi^{-}$and $\pi^{+}$at all other energies, the $A_{y}$ are mostly consistent with zero. These data differ from the predictions of present pion-nucleus reaction theories, especially at large momentum transfers.
\end{abstract}

PACS numbers: $25.80 . \mathrm{Dj}, 24.70 .+\mathrm{s}$

Since significant nuclear polarizations can now be achieved by the dynamic nuclear-polarization (DNP) technique, ${ }^{1}$ it has become possible to measure the leftright asymmetries or analyzing powers $A_{y}$ in pion scattering from nuclear targets. ${ }^{2-4}$ Measurements of $A_{j}$ allow sensitive tests of $\pi$-nucleus interaction models ${ }^{2.5,6}$ which include medium modifications of the free pionnucleon interaction in nuclei. In addition, such measurements contain information on the isoscalar spin-flip strength of nuclear transitions which is difficult to obtain otherwise.

A recent experiment at the Paul Scherrer Institute (PSI) by Tacik et al. ${ }^{4}$ and Meier et al. ${ }^{7}$ on ${ }^{15} \mathrm{~N}\left(\pi^{+}, \pi^{+}\right)$ at $T_{n}=164 \mathrm{MeV}$ revealed unexpectedly small asymmetries in sharp contrast to the large $A_{y}$ predicted by theory. In this Letter we report $A_{y}$ measurements on polarized ${ }^{13} \mathrm{C}$. Angular distributions of $A_{y}$ and of differential cross sections $d \sigma(\theta) / d \Omega$ were measured for both $\pi^{-}$and $\pi^{+}$at one energy below $(132 \mathrm{MeV})$ and one above (about $230 \mathrm{MeV}$ ) the $P_{33}$ resonance. At $T_{\pi}=132$ $\mathrm{MeV}$, the $A_{y}$ of $\pi^{-}$scattering were found to be nonzero near the angular regions corresponding to the first and the second minima of the differential cross sections. A survey was also made at several energies (114-180 $\mathrm{MeV})$ across the resonance for $\pi^{-}$scattering to search for predicted large $A_{y}$ at momentum transfers $(q)$ near the second minimum of $d \sigma(\theta) / d \Omega$, but $A_{y}$ consistent with zero were found.

The scattering amplitude of pion scattering from a spin- $\frac{1}{2}$ nucleus can be expressed as

$$
t=f(\theta)+i g(\theta) \hat{\mathbf{n}} \cdot \sigma,
$$

where $\sigma$ is the nuclear Pauli spin matrix, $\hat{\mathbf{n}}=\left(\mathbf{k} \times \mathbf{k}^{\prime}\right) /$ $\left|\mathbf{k} \times \mathbf{k}^{\prime}\right|$ with $\mathbf{k}$ and $\mathbf{k}^{\prime}$ being the momenta of the incoming and outgoing pions, and $f(\theta)$ and $g(\theta)$ are, respectively, the spin-independent ànd spin-dependent pion-nucleus scattering amplitudes. The former proceeds without a spin transfer $(\Delta S=0)$ to the nucleus; the latter involves a spin transfer $(\Delta S=1) . d \sigma(\theta) / d \Omega$ for pion elastic scattering from nuclei with nonzero spin is dominated by the spin-independent amplitude. A contribution from the smaller spin-dependent amplitude is usually difficult 
to observe since $d \sigma(\theta) / d \Omega=|f(\theta)|^{2}+|g(\theta)|^{2}$. However, the left-right asymmetry, defined as

$A_{y}=\frac{(d \sigma / d \Omega)_{1}-(d \sigma / d \Omega)_{1}}{(d \sigma / d \Omega)_{1}+(d \sigma / d \Omega)_{1}}=\frac{2 \operatorname{Im}\left[f(\theta) g(\theta)^{*}\right]}{|f(\theta)|^{2}+|g(\theta)|^{2}}$

involves the interference of $f(\theta)$ and $g(\theta)$, and is therefore sensitive to the spin-dependent scattering amplitude.

Pion elastic scattering is more sensitive to the isoscalar than the isovector part of the interaction. Thus, our data complement measurements of the (isovector-dominated) magnetic form factor obtained from electron scattering. By measuring both $\pi^{+}$and $\pi^{-}$scattering, we also obtain information on the isovector terms. As mentioned above, asymmetry data should provide tests of models of the pion-nucleus interaction. Indeed, inclusion of secondorder terms in the theoretical calculations ${ }^{2.5 .6}$ provides larger changes in the predicted $A_{y}$ than in $d \sigma(\theta) / d \Omega$.

The experiment was carried out at the Clinton P. Anderson Meson Physics Facility (LAMPF). The material of the polarized target was $99 \%{ }^{13} \mathrm{C}$-enriched 1 -butanol $\left({ }^{13} \mathrm{C}_{4} \mathrm{H}_{10} \mathrm{O}\right)$ in the form of frozen beads of about $1 \mathrm{~mm}$ in diameter. The beads were contained in a cylindrical Teflon cell immersed in a liquid- ${ }^{3} \mathrm{He}$ bath inside a copper microwave cavity. The temperature of the target was kept below $0.5 \mathrm{~K}$. The target was polarized either up or down with respect to the reaction plane by ${ }^{1-3}$ DNP in a magnetic field of $2.5 \mathrm{~T}$. Polarizations of ${ }^{13} \mathrm{C}$ and ${ }^{1} \mathrm{H}$ were measured by a nuclear-magnetic-resonance system. The polarizations were calibrated by measuring the thermal equilibrium ${ }^{3}$ signals periodically. The average polarization of ${ }^{13} \mathrm{C}$ was about $28 \%$. A thin ${ }^{13} \mathrm{C}$ slab target was used to collect additional differential-crosssection data.

The left-right asymmetries were obtained by detecting the pions scattered from the polarized target with opposite spin orientations at the same scattering angles. The outgoing pions were detected by the Large Acceptance Spectrometer. ${ }^{8.9}$ The angular acceptance of $8^{\circ}$ was divided into two $4^{\circ}$ angular bins for the angular-distribution data (Fig. 2) and into three $2.7^{\circ}$ angular bins for the energy-dependence data (Fig. 3). The energy resolution was 2-3 MeV (FWHM) (Fig. 1), limited primarily by target thickness and inhomogeneity. Contributions from the unresolved $3.09-\mathrm{MeV}$ peak are expected to be very small ${ }^{10}$ even at the minima of the elastic cross sections. The tails from the unresolved $3.68 / 3.85-\mathrm{MeV}$ doublet make only small contributions in the region of the elastic peak.

In terms of measurable quantities, the asymmetry of pion scattering from polarized ${ }^{13} \mathrm{C}\left(J=\frac{1}{2}\right)$ is

$$
A_{y}=\frac{N_{1}-N_{1}}{\left(N_{1}-B\right) P_{1}+\left(N_{1}-B\right) P_{1}}=\frac{\text { DIFF }}{\text { SUM }} .
$$

Here $P_{1}$ and $P_{1}$ are the transverse polarizations of the ${ }^{13} \mathrm{C}$ target with spin orientation parallel (spin up) and antiparallel (spin down) to $\hat{\mathbf{n}}$. $N_{1}$ and $N_{1}$ are the corresponding scattering yields. The background $B$ in the region of the elastic peak originated from the target container (copper microwave cavity and Teflon cell). and from the oxygen in the butanol. A two-step subtraction was made (Fig. 1) using data from a replica of the cryogenic target but without the butanol and ${ }^{3} \mathrm{He}$, and data from this replica filled with $\mathrm{H}_{2} \mathrm{O}$.

We extracted $A_{y}$ by first generating spectra of $A_{y}$ [Eq. (3)] and its uncertainty $\Delta A_{y}$. Errors in background subtraction in the DIFF spectra are primarily statistical. Small nonstatistical uncertainties may arise from the relative normalizations. Larger nonstatistical uncertainties exist in the background subtraction when generating the SUM spectra, for example, due to the difference in peak shapes. However, the effect of these uncertainties on $\Delta A_{y}$ is small because $\Delta A_{y}$ is dominated by the relatively poor statistics in DIFF spectra. A statistically. weighted average of $A_{y}$ was calculated. Consistent results within error bars were attained when the asymmetry was calculated either from the channels within the half-width of the elastic peak, or slightly larger regions, or from using a peak-fitting method. (At all angles the elastic peak was clearly visible in the SUM spectra.)

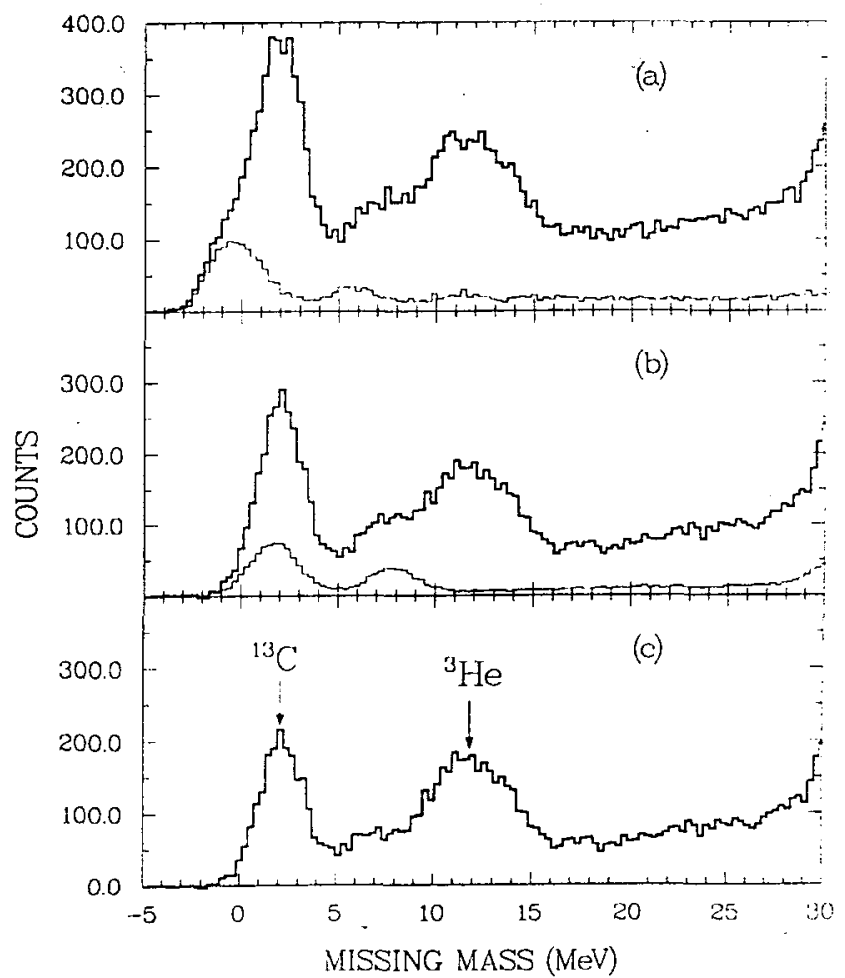

FIG. 1. Typical foreground- and background-subtracted missing-mass spectra for pion scattering from polarized ${ }^{13} \mathrm{C}_{4} \mathrm{H}_{10} \mathrm{O}$ : (a) thick line, full spectrum; thin line, $\mathrm{Cu}$ background spectrum; (b) thick line, spectrum with $\mathrm{Cu}$ background subtracted; thin line. oxygen background spectrum; (c) thick line, spectrum with $\mathrm{Cu}+{ }^{16} \mathrm{O}$ background subtracted. 
The angular distributions of $A_{y}$ at $T_{\pi}=132 \mathrm{MeV}$ for both $\pi^{-}$and $\pi^{+}$scattering from this experiment are presented in Fig. 2. Nonzero $A_{y}$ were observed for $\pi^{-}$ scattering at $T_{\pi}=132 \mathrm{MeV}$ at angles near the first and second minima of $d \sigma(\theta) / d \Omega, \approx 60^{\circ}$ and $\approx 100^{\circ}$, respectively, whereas for $\pi^{+}$scattering the $A_{y}$ were consistent with zero at all angles. A larger $A_{y}$ is expected and observed for $\pi^{-}$scattering than for $\pi^{+}$scattering because there is an excess neutron in ${ }^{13} \mathrm{C}$ to which a $\pi^{-}$ couples more strongly than a $\pi^{+}$in the region of the $P_{33}$ resonance. ${ }^{11}$ Measurements were also done at $T_{\pi}=226$ $\mathrm{MeV}$ for $\pi^{+}$and at $T_{\pi}=231 \mathrm{MeV}$ for $\pi^{-}$. Asymmetries (not shown) consistent with zero were found for both $\pi^{+}$ and $\pi^{-}$in the angular range of $40^{\circ}$ to $80^{\circ}$ at these energies.

The $A_{y}$ from the search for predicted large values in $\pi^{-}$scattering near the second minimum of $d \sigma(\theta) / d \Omega$, $1.75<q<2.05 \mathrm{fm}^{-1}$, were all found to be very small (Fig. 3). Specifically, at $T_{n}=165 \mathrm{MeV}$ our data are consistent with zero, a result similar to that found in the experiment ${ }^{4}$ on ${ }^{15} \mathrm{~N}$.

The error bars shown in Figs. 2 and 3 include only the statistical and background subtraction errors. Systematic errors in deriving the yields of the scattered pions (due to the uncertainties in the absolute beam flux, target thickness, and solid angle) cancel in the calculation of $A_{y}$ [Eq. (3)]. The uncertainty in the determination of the target polarization was estimated to be $3 \%$.

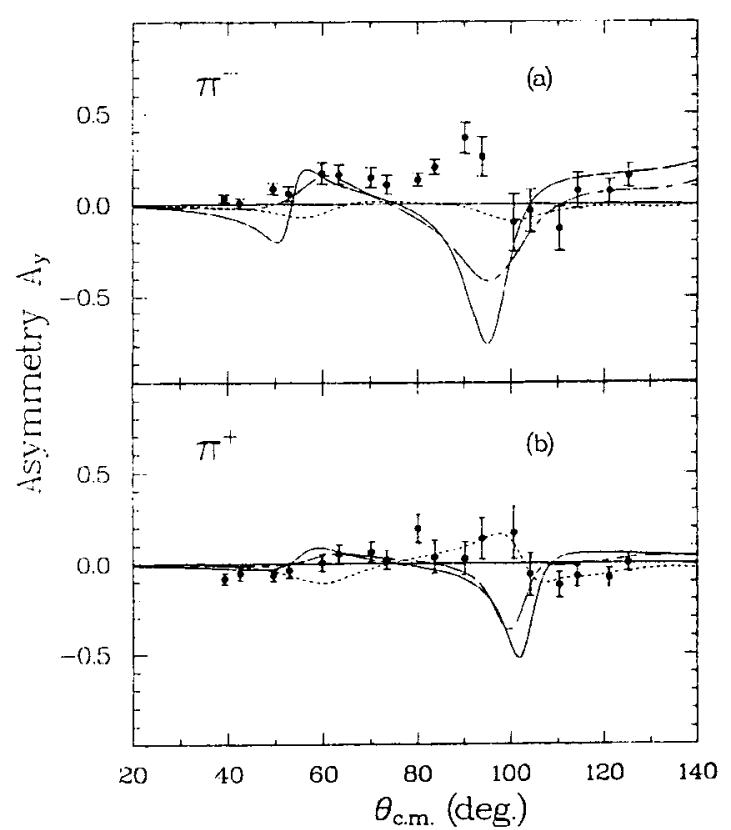

FIG. 2. Asymmetries $A_{y}$, for elastic scattering of (a) $\pi^{-}$and (b) $\pi^{+}$from ${ }^{13} \overrightarrow{\mathrm{C}}$ at $T_{\pi}=132 \mathrm{MeV}$. The curves are from DWIA calculations of this work (solid lines) and from predictions of Mach (Ref. 6) with densities due to Tiator (Ref. 17) (dashed lines) and with CK (Refs. 14 and 15) densities (chain-dashed lines).
In an attempt to reproduce the data, we have calculated ${ }^{12}$ theoretical $A_{y}$ using a model which employs a firstorder optical potential. We used the optical-model program ${ }^{13}$ PIPIT (which does not include spin transfer) in conjunction with the inelastic-scattering code ARPIN. ${ }^{14}$ The spin-independent and spin-dependent parts of the elastic transition amplitude were obtained from ARPIN, and $d \sigma(\theta) / d \Omega$ and $A_{y}$ were calculated in the distortedwave impulse approximation (DWIA) with the distorted waves from PIPIT. The pion-nucleon $t$ matrix was calculated at an energy below the actual pion-nucleon centerof-mass energy which appears to correct for some second-order effects. We found that at $T_{\pi}=132 \mathrm{MeV}$ an energy shift of $14 \mathrm{MeV}$ for $\pi^{+}$and of $4 \mathrm{MeV}$ for $\pi^{-}$ gave better fits with the elastic-scattering cross sections than the $20-\mathrm{MeV}$ shift used ${ }^{10}$ at $162 \mathrm{MeV}$. We chose the difference of $10 \mathrm{MeV}$ between the values for $\pi^{+}$and $\pi^{-}$to be of the order of the $\pi$-nucleus Coulomb energy differences at the nuclear surface. A Gaussian off-shell model ${ }^{13}$ was employed with a momentum range parameter ${ }^{12,13}$ of $3 \times 10^{-6} \mathrm{MeV}^{-2}$ at all energies.

At small momentum transfers $q$ (scattering angles below $70^{\circ}$ ), where the Cohen-Kurath (CK) model ${ }^{15}$ provides us with reliable ground-state (g.s.) spin transition densities, we found reasonable agreement between the data at $132 \mathrm{MeV}$ and our calculations (Fig. 2, solid lines). However, at large $q$ (scattering angles of $\approx 100^{\circ}$ ), these calculations give incorrect signs for $A_{y}$. This failure is not unexpected because at large $q$ the $A_{y}$ depend strongly on both the reaction mechanism and the nuclear-structure model. We note that standard nuclear-structure models have thus far failed to reproduce

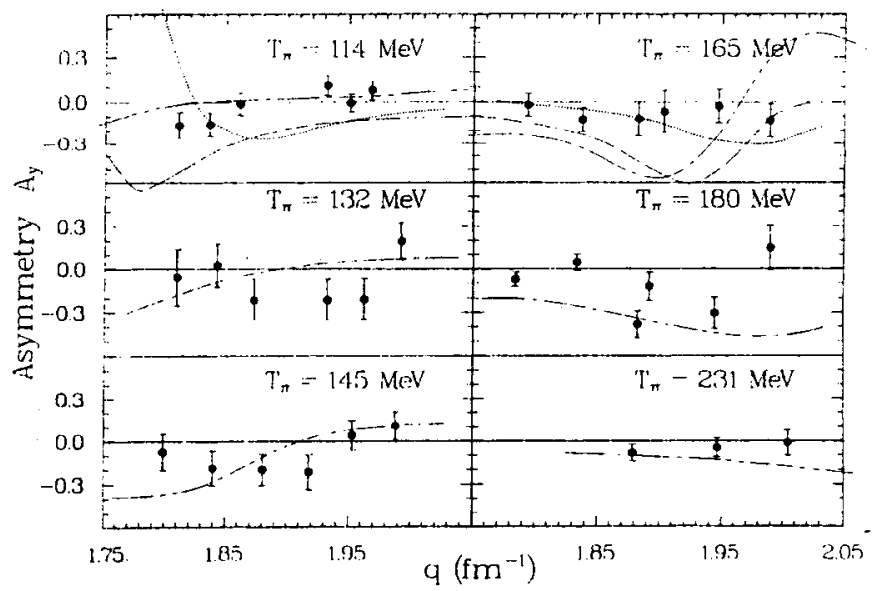

FIG. 3. $A$, for elastic.scattering of $\pi^{-}$from ${ }^{13} \mathrm{C}$ at energies across the $[3,3]$ resonance at momentum transfers near the second minimum of the differential cross sections. The curves at 114 and $165 \mathrm{MeV}$ are the predictions of Thies using the $\Delta$ hole model (Ref. 5) (dotted lines) and the closure approximation to this model (chain-dotted lines). The chain-dashed lines are calculations of Mach (Ref. 6) with CK (Refs. 14 and 15) densities. 
the measured magnetic form factor ${ }^{16}$ for $q>1.6 \mathrm{fm}^{-1}$.

With the $C K$ densities, Mach $^{6}$ provided a prediction (chain-dashed curves in Figs. 2 and 3), based on a firstorder optical potential as above, but including a phenomenological $\rho^{2}$ term which was adjusted to fit $d \sigma(\theta) / d \Omega$ at various energies. Here $\rho$ is the nuclear density. Mach's predictions yield values of $A_{y}$ similar to our calculations except at $\theta_{\text {c.m. near }} 55^{\circ}$. The fits with the elastic cross sections are generally better in the calculations of Mach than in our calculations, which predict a deeper first minimum than observed experimentally.

Additional calculations (dashed lines in Fig. 2) were performed by Mach with the same reaction model, but with densities provided by Tiator. ${ }^{17}$ These densities had been derived in an attempt to reproduce the anomalously low cross sections for photoproduction of charged pions on ${ }^{13} \mathrm{C}$. The DWIA curves from these calculations are in disagreement with the data around $60^{\circ}$ and also at the larger angles for $\pi^{-}$; for $\pi^{+}$a small peak is predicted at $95^{\circ}$ which is consistent with the data although the error bars do not rule out zero $A_{y}$.

The large differences between the results of these calculations with the Tiator densities ${ }^{17}$ (dashed curves) and the CK densities ${ }^{14.15}$ (chain-dashed curves) demonstrate a strong dependence of $A_{y}$ on the nuclear-structure model. However, the $d \sigma(\theta) / d \Omega$ (for both $\pi^{-}$and $\pi^{+}$) calculated with the two densities are almost identical. A wave-function dependence of $A_{y}$ was also found in work ${ }^{18}$ on ${ }^{6} \mathrm{Li}$.

The small $A_{y}$ observed in the energy-dependence data and some theoretical predictions are shown in Fig. 3. At 165 and $114 \mathrm{MeV}$ the data disagree with the theoretical curves obtained with $\mathrm{CK}$ densities ${ }^{14,15}$ by Mach (chaindashed curves) and very similar ones obtained by us (not shown). $\Delta$-hole model predictions of Thies and coworkers ${ }^{5}$ are also available at some of these energies and shown as dotted lines (full calculation) and chain-dotted lines (closure approximation). The wave functions used in these two $\Delta$-hole calculations are again different from those of CK and Tiator. But the large difference in the predicted $A_{y}$ (Fig. 3) from two different approaches to the reaction mechanism shows that the $A_{y}$ are sensitive to the reaction model used. At $165 \mathrm{MeV}$ the data show a preference for the closure approximation calculation. $\Delta$-hole model calculations are not yet available at the other energies.

Further data on polarized ${ }^{13} \mathrm{C}$ have been taken at 100 $\mathrm{MeV}$ at TRIUMF (Ref. 19) and at $162 \mathrm{MeV}$ at LAMPF. ${ }^{20}$ Both data sets are presently being analyzed.

The asymmetry data of this and other recent work ${ }^{4.18}$ are not yet understood theoretically. They contain new information on the nuclear spin transition density and the pion-nucleus reaction mechanism. Further theoretical effort is needed to describe the high- $q$ behavior of the nuclear transition density, and specifically, its isoscalar spin-dependent part. Once that task is accomplished, our data may provide tests of pion-nucleus interaction theories which include higher-order terms such as the $\Delta$ nucleus spin-orbit force.

We wish to thank Dr. R. Mach and Dr. M. Thies for permission to present the results of their theoretical calculations and Dr. P. J. Ellis for a critical reading of the manuscript. We also acknowledge a grant for computing time from the Minnesota Supercomputer Institute. This work was supported in part by the U. S. Department of Energy, the University of Minnesota Graduate School, and the Robert A. Welch Foundation.

(a) Present address: Universität Karlsruhe, D-7500 Karlsruhe, Germany, and Paul Scherrer Institute, $\mathrm{CH}-5234$ Villigen, Switzerland.

(b) Present address: University of New Mexico, Albuquerque, NM 87131.

(c) Present address: Department of Physics, Old Dominion University, Norfolk, VA 23529.

(d) Present address: EG \&G/EM, 130 Robin Hill Road, Goleta, CA 93116.

(e) Present address: MS 1049, Superconducting Super Collider (SSC) Laboratory, 2550 Beckleymeade Avenue, Dallas, TX 75237.

'A. Abragam and M. Goldman, Nuclear Magnetism: Order and Disorder (Oxford Univ. Press, New York, 1982), Chap. 6.

${ }^{2}$ See, e.g., Proceedings of the LAMPF Workshop on Physics with Polarized Nuclear Targets, edited by G. Burleson et al. (Los Alamos National Laboratory Report No. LA-10772-C. 1986).

3J. J. Jarmer et al, Nucl. Instrum. Methods Phys. Res., Sect. A 250, 576 (1986).

${ }^{4} R$. Tacik et al., Phys. Rev. Lett. 63, 1784 (1989).

${ }^{5} \mathrm{M}$. Thies (private communication); M. Hirata, F. Lenz, and M. Thies, Phys. Rev. C 28, 785 (1983).

${ }^{6} \mathrm{R}$. Mach (private communication); R. Mach and S. S. Kamalov, Nucl. Phys. A511, 601 (1990).

${ }^{7} \mathrm{R}$. Meier et al., Phys. Rev. C 42, 2222 (1990).

${ }^{8}$ E. Colton, Nucl. Instrum. Methods 178, 95 (1980).

${ }^{9}$ A. L. Williams et al., Phys. Lett. B 216, 11 (1989).

${ }^{10}$ S. J. Seestrom-Morris et al., Phys. Rev. C 26, 594 (1982).

"C. L. Morris et al., Phys. Rev. C 17, 227 (1978); D.

Dehnhard et al., Phys. Rev. Lett. 43, 1091 (1979).

${ }^{12} \mathrm{~S}$. Chakravarti et al., University of Minnesota report (unpublished).

${ }^{13}$ R. A. Eisenstein and F. Tabakin, Comput. Phys. Commun. 12, 237 (1976).

${ }^{14}$ T.-S. H. Lee and D. Kurath, Phys. Rev. C 21, 293 (1980).

${ }^{15}$ S. Cohen and D. Kurath, Nucl. Phys. A226, 253 (1974).

${ }^{16}$ R. S. Hicks et al., Physs. Rev. C 26, 339 (1982).

${ }^{17}$ L. Tiator, Phys. Lett. 125B, 367 (1983).

${ }^{18}$ S. Ritt el al., Phys. Rev. C 43, 745 (1991).

${ }^{19}$ TRIUMF Experiment No. 504 (unpublished).

${ }^{20}$ LAMPF Experiment No. 1025 (unpublished). 


\title{
Analyzing Powers for Pion Charge Exchange on Polarized ${ }^{13} \mathrm{C}$
}

\author{
J. J. Görgen, J. R. Comfort, J. R. Tinsley, ${ }^{(a)}$ T. Averett, J. DeKorse, B. Franklin, and B. G. Ritchie \\ Physics Department, Arizona State University, Tempe, Arizona 85287 \\ G. Kyle, A. Klein, ${ }^{(b)}$ B. Berman, ${ }^{(c)}$ G. Burleson, and K. Cranston
}

Physics Department, New Mexico State University, Las Cruces, New Mexico 88003

J. A. Faucett, J. J. Jarmer, J. N. Knudson, S. Penttilä, and N. Tanaka

Los Alamos National Laboratory, Los Alamos, New Mexico 87545

B. Brinkmöller, ${ }^{(d)}$ D. Dehnhard, and Yi-Fen Yen

Physics Department, Unicersity of Minnesota, Minneapolis, Minnesota 55455

S. Høibråten

Department of Physics, University of Colorado, Boulder, Colorado 80309

\author{
H. Breuer, B. S. Flanders, ${ }^{(e)}$ M. A. Khandaker, D. L. Naples, and D. Zhang \\ Department of Physics and Astronomy, University of Maryland, College Park, Maryland 20742 \\ M. L. Barlett, ${ }^{())}$G. W. Hoffmann, and M. Purcell \\ Department of Physics. The University of Texas, Austin. Texas 78712 \\ (Received 13 September 1990)
}

\begin{abstract}
Results of the first pion single-charge-exchange experiment on a polarized nuclear target are reported. Analyzing powers $A_{y}$ for the $\left(\pi^{+}, \pi^{0}\right)$ reaction at $T_{\pi^{+}}=163 \mathrm{MeV}$ on a polarized ${ }^{13} \mathrm{C}$ target were measured over an angular range between $20^{\circ}$ and $60^{\circ}$ in the laboratory system. Calculations in the distorted-wave impulse approximation do not reproduce these new data.

PACS numbers: $25.80 . \mathrm{Fm}, 24.10 . \mathrm{Eq}, 24.70 .+\mathrm{s}, 27.20 .+\mathrm{n}$
\end{abstract}

We report first results arising from a new approach to the study of isovector spin-dependent interactions of pions in nuclei. These results are obtained from a measurement of the analyzing power of the $\left(\pi^{+}, \pi^{0}\right)$ reaction on a transwersely polarized ${ }^{13} \mathrm{C}$ target at a beam energy of $163 \mathrm{MeV}$.

Parity and rotational invariance determine the elementary pion-nucleon interaction $t$ matrix to be of the form

$$
t_{b a}=f(\theta)+i g(\theta) \hat{\mathbf{n}} \cdot \boldsymbol{\sigma},
$$

where $\hat{\mathbf{n}}=\left(\mathbf{k} \times \mathbf{k}^{\prime}\right) /\left|\mathbf{k} \times \mathbf{k}^{\prime}\right|$ ( $\mathbf{k}$ and $\mathbf{k}^{\prime}$ are the momenta of the incident and outgoing pions), and $\sigma$ is the Pauli spinor for the nucleon. The complex amplitudes $f(\theta)$ and $g(\theta)$ each have isoscalar and isovector parts. Elastic pion scattering is primarily sensitive to the isoscalar terms, and analyzing powers for $\pi^{ \pm}$elastic scattering at energies near the $P_{33}$ resonance from polarized protons ${ }^{1}$ and polarized nuclei in the $1 p$ shell $^{2}$. have recently been reported. Although the asymmetries from the elementary process are sizable, those for spin- $\frac{1}{2}$ nuclear targets were found to be small. ${ }^{2.3}$ The interpretation of these results is not yet clear.

There is currently very little information on the isovector part of $g(\theta)$ since pion single-charge-exchange (SCX) cross sections at forward angles are dominated by the isovector part of $f(\theta)$ and no experiments to date have isolated a transition dominated by spin transfer. However, SCX reactions on targets with spin create new possibilities. In the case of spin- $\frac{1}{2}$ targets, the pionnucleus amplitude may be written in a form similar to Eq. (1), but with effective-medium-modified amplitudes $F(\theta)$ and $G(\theta)$ replacing the elementary ones. In addition to cross sections, the amplitudes yield analyzing powers given by (with $100 \%$ target polarization)

$$
A_{y}(\theta)=\frac{(d \sigma / d \Omega)_{1}-(d \sigma / d \Omega)_{1}}{(d \sigma / d \Omega)_{1}+(d \sigma / d \Omega)_{!}}=\frac{2 \operatorname{Im}\left(F G^{*}\right)}{|F(\theta)|^{2}+|G(\theta)|^{2}}
$$

where the arrow indicates the nucleon spin direction relative to $\hat{n}$ and the denominator is equal to the spinaveraged cross section. It is important to note from Eq. (2) that the analyzing power $A_{y}$ is sensitive to the important phase relationship between $F(\theta)$ and $G(\theta)$. Our experiment provides information on the isovector part of that phase in the nuclear medium for the first time.

The ${ }^{13} \mathrm{C}$ spin-parity of $\frac{1}{2}-$ keeps the number of spindependent observables to a minimum and therefore represents a sound starting point for exploring pion-nucleus spin physics. The isobaric-analog state (IAS) transition to the ground state of ${ }^{13} \mathrm{~N}$ has minimal background, and the ${ }^{13} \mathrm{C}\left(\pi^{+}, \pi^{0}\right)$ unpolarized cross sections have been 
previously measured at $165 \mathrm{MeV}^{4}$

Measurements were made at the low-energy pion channel at the Clinton P. Anderson Meson Physics Facility (LAMPF) with the LAMPF $\pi^{0}$ spectrometer $^{5}$ which was positioned at laboratory scattering angles of $25^{\circ}$. $38^{\circ}$, and $55^{\circ}$. It was set at a distance of $1.5 \mathrm{~m}$ from the target for the $25^{\circ}$ measurements and at $1.0 \mathrm{~m}$ for the $38^{\circ}$ and $55^{\circ}$ measurements. The angular acceptance was about $24^{\circ}$ at the $1-\mathrm{m}$ setting and about $18^{\circ}$ at 1.5 $m$. The angular resolution of the spectrometer was about $6^{\circ}$, and two or three separate angles were binned from the data at each spectrometer setting.

The target material consisted of about $10 \mathrm{~cm}^{3}$ of frozen beads, about $1.5 \mathrm{~mm}$ in diameter, composed of ethylene glycol $\mathrm{OH}-\left(\mathrm{CH}_{2}\right)_{2}-\mathrm{OH}$ doped with $7 \times 10^{19}$ molecules/m! of EHBA.Cr ${ }^{(v)} 6$ The carbon was enriched to $99 \%{ }^{13} \mathrm{C}$ atoms. The beads were contained in a Teflon basket which was placed inside a thin-walled copper cell. The cell was $2 \mathrm{~cm}$ thick and the effective thickness of the carbon was about $620 \mathrm{mg} / \mathrm{cm}^{2}$. The target cell was cooled to about $0.5 \mathrm{~K}$ with a ${ }^{3} \mathrm{He}$ evaporation refrigerator. A polarization normal to the scattering plane of about $27 \%$ was induced by using the dynamic nuclear polarization technique ${ }^{7}$ in a uniform magnetic field of $2.5 \mathrm{~T}$. The target polarization was measured with the nuclear-magnetic-resonance (NMR) method. The NMR signal was normalized by using the thermal equilibrium technique. ${ }^{\text {? }}$

The relative $\pi^{+}$beam intensity was measured by means of two toroidal current monitors through which the primary proton beam passed and by an ion chamber through which the $\pi^{+}$beam passed. The absolute beam intensity was determined periodically by measuring the " $\mathrm{C}(\beta)$ activity produced in thin scintillator disks. ${ }^{8}$ It was helpful for comparisons of the derived cross sections with those from Ref. 4, but was not needed for the analyzing powers.

Backgrounds arising from accidental two-photon coincidences and from the cryostat were subtracted from the raw data. The background spectra were obtained by-using a replica of the real polarized target with water replacing the ethylene glycol target beads and the ${ }^{3} \mathrm{He}$ refrigerant. The yield due to non- ${ }^{13} \mathrm{C}$ contaminants in the IAS region is small, due to a favorable $Q$ value for the IAS transition of $+2.89 \mathrm{MeV}$; the nearest significant contaminant reaction ${ }^{3} \mathrm{He}\left(\pi^{+}, \pi^{0}\right) 3 p$ is separated from the IAS by $4.7 \mathrm{MeV}$. The spectra were then analyzed by fitting one or more peaks, along with background functions, to the low-excitation region. The shape of the peak was determined from a Monte Carlo simulation of the entire experimental arrangement and it correctly reproduces the signal shape observed with the same experimental apparatus for the elementary $\pi^{-} p \rightarrow \pi^{0} n$ reaction. ${ }^{9}$ The peak shape had a full width at half maximum of about $5 \mathrm{MeV}$.

Figure 1 shows spectra for detected events with $X=\left|E_{1}-E_{2}\right| /\left(E_{1}+E_{2}\right) \leq 0.15$, where $E_{1}$ and $E_{2}$ are

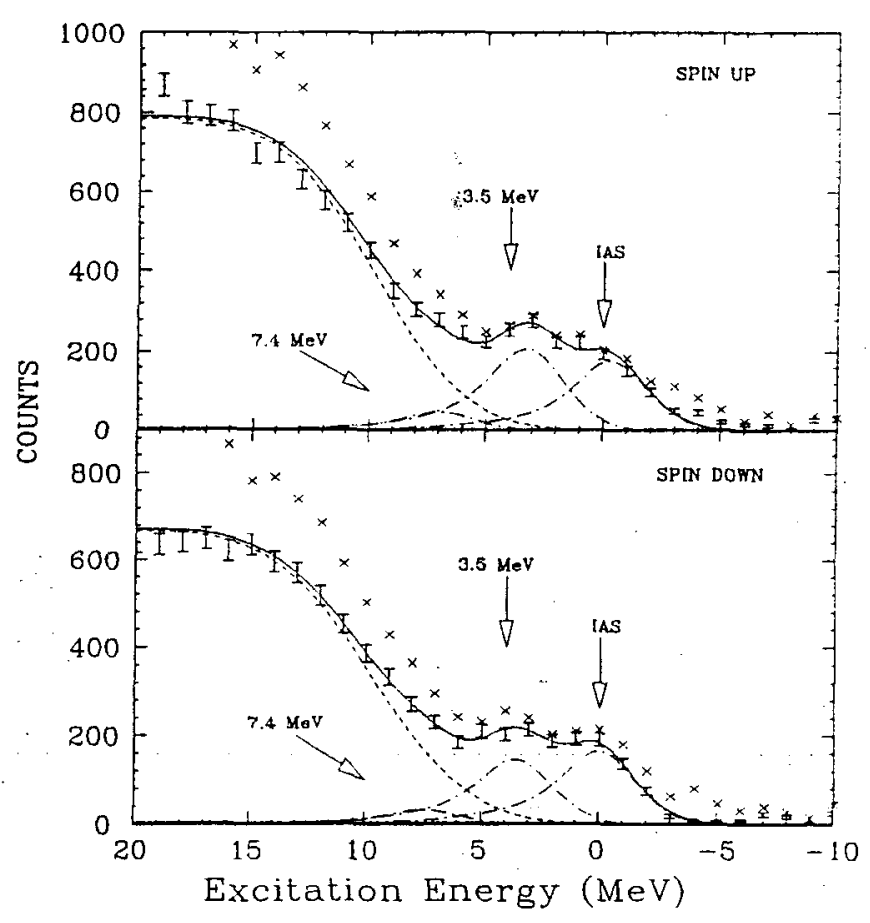

FIG. 1. Spectra for the ${ }^{13} \overrightarrow{\mathrm{C}}\left(\pi^{+}, \pi^{0}\right)$ reaction at $163 \mathrm{MeV}$ and at $22.4^{\circ}$. The solid curve is the fitted sum of three peaks shown individually as dash-dotted curves. The dashed curve represents the fitted nuclear background. The crosses represent the data prior to subtraction of the instrumental background.

the laboratory energies of the two photons resulting from the $\pi^{0}$ decay. ${ }^{5}$ Structure that can be associated with three separate states is evident. The spectra cannot be well described with fits based on the assumption of a single peak with a phenomenological background, and the extracted cross sections were typically less than those reported in an earlier experiment. ${ }^{4}$ The kinematics of such a peak were also inconsistent with ${ }^{13} \mathrm{C}$. However, good fits were obtained by including peaks for two excited states as well. The positions of the three peaks were found to follow the kinematics of the ground state and states near $3.5-$ and $7.4-\mathrm{MeV}$ excitation in ${ }^{13} \mathrm{C}$. The summed cross sections of the ground state and $3.5-\mathrm{MeV}$ state are in good agreement with the earlier results. ${ }^{4}$

The features seen in Fig. 1 were present in all of the other spectra as well. Separate fits were made to the spin-up and spin-down spectra. We assumed that the same shape of the background, adjusted only in normalization, could be used for both spin orientations of each angle bin. Various phenomenological forms for the background were tested. A third-order polynomial form produced results that agreed with those obtained with the adopted exponential-plus-constant form. Both of these forms had essentially zero magnitudes at the ${ }^{13} \mathrm{~N}$ proton-decay threshold of $1.94 \mathrm{MeV}$.

The summed yields from the spin-up and spin-down 
spectra agree with the yields obtained from independent fits to the summed spectra. The events in the empirical difference spectra were too few to be useful for fittirig directly. However, the differences in the fits to the spinup and spin-down spectra were consistent with these difference spectra.

The analyzing powers were computed from the expression

$$
A_{y}(\theta)=\left(N^{+}-N^{-}\right) /\left(N^{+} P^{-}+N^{-} P^{+}\right),
$$

where $N^{+}\left(N^{-}\right)$is the normalized yield of good charge-exchange events with the target polarized parallel (antiparallel) to the normal to the scattering plane, and $P^{+}$and $P^{-}$are the corresponding target polarization values. The features of the analyzing powers were stable to changes in details of the fitting procedure such as the forms of the background and constraints on the absolute or relative positions of the peaks. Fits that included the $\frac{1}{2}^{+}$state at $2.36 \mathrm{MeV}$ gave no statistical improvement and were otherwise unsatisfactory. This state and its analog in ${ }^{13} \mathrm{C}$ are known to be very weakly populated in inelastic-scattering and charge-exchange reactions. ${ }^{10-12}$ The observed first-excited state corresponds to the collectively enhanced $\frac{3}{2}^{-}, \frac{5}{2}^{+}$doublet separated by about 3.5 $\mathrm{MeV}$ from the IAS. The peak at the best-fit excitation energy of $7.4 \mathrm{MeV}$ involves transitions to several states.

The experimental results are shown in Figs. 2 and 3. The cross sections for the excited state are comparable to

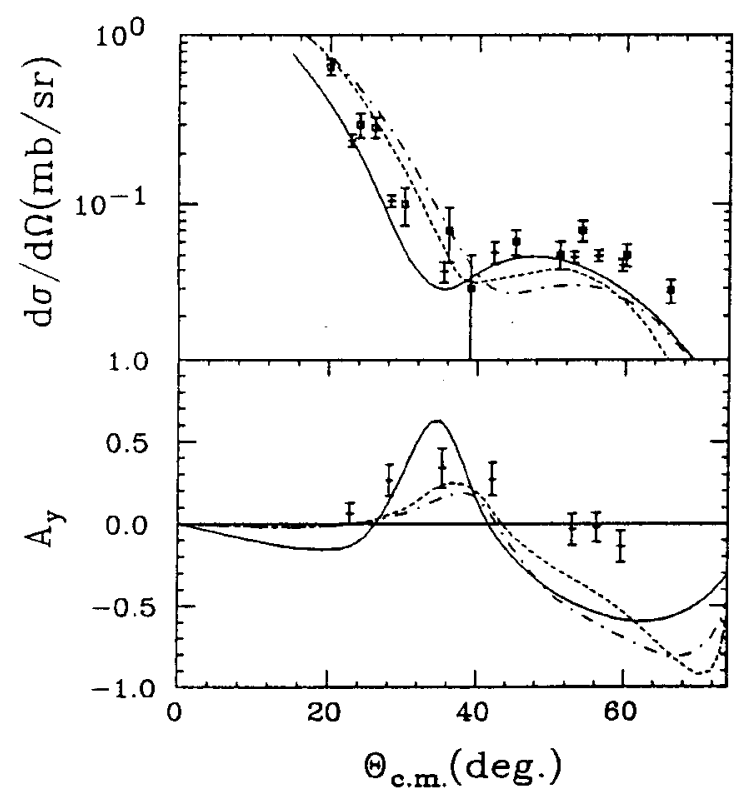

FIG. 2. Cross sections and analyzing powers for the ${ }^{13} \overrightarrow{\mathrm{C}}\left(\pi^{+}, \pi^{0}\right)$ reaction at $163 \mathrm{MeV}$ to the isobaric-analog state. The cross sections from Doron et al. (Ref. 4) are shown as open squares. The curves are results of DWIA calculations by Siegel (solid), and by Mach (CK wave functions, dashed; Tiator wave functions, dot-dashed). those for the IAS, but differ in their angular dependence. The analyzing powers for the two states are quite different. At this time the relative contributions of the $\frac{3}{2}^{-}$and $\frac{5}{2}^{+}$states to the yields for the $3.5-\mathrm{MeV}$ group are unknown.

The error bars include statistical and fitting uncertainties associated with the determination of $N^{+}$and $N^{-}$. as well as uncertainties arising from background subtractions. The fitting uncertainty was estimated from the results obtained from variations of the peak-fitting procedures and ranged from 0.05 to 0.11 . Many of the uncertainties that apply to differential cross sections cancel out in the expression for $A_{y}(\theta)$. An estimate of the systematic uncertainty of $A_{y}$ for each angle setting of the spectrometer was obtained from comparisons of the analyzing powers extracted from sets of runs with the same polarization orientation. False (nonzero) asymmetries can arise from fluctuations in the ion chamber and beam-toroid counters used to monitor the relative beam flux, fluctuations in the steering of the beam, and statistical uncertainties associated with the determination of the target polarization. Such systematic errors of 0.04-0.06 were combined in quadrature with the statistical errors associated with Eq. (3). Not included in Figs. 2 and 3 is an additional systematic uncertainty in the target polarization, estimated to be about $4.0 \%$. This uncertainty arises from possible errors in the temperature calibration for the thermal equilibrium measurements as well as from biases in estimating the back-

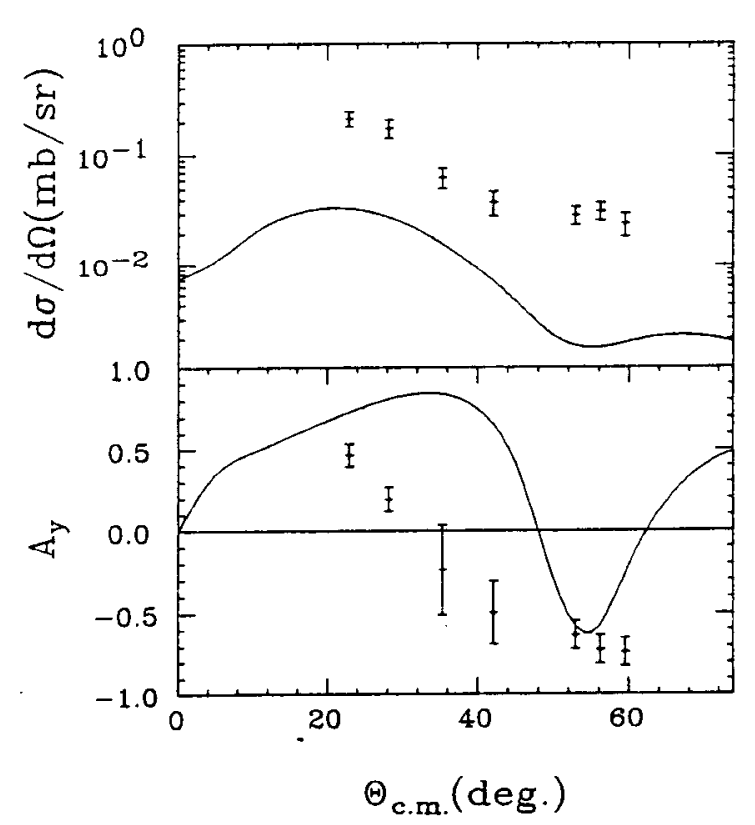

FIG. 3. Cross sections and analyzing powers for the ${ }^{13} \overrightarrow{\mathrm{C}}\left(\pi^{+}, \pi^{0}\right)$ reaction at $163 \mathrm{MeV}$ to the $\frac{1}{2}^{-}, \frac{5}{2}^{+}$doublet at 3.5 $\mathrm{MeV}$. The solid curves, for the $\frac{3}{2}^{-}$state only, have the same meaning as in Fig. 2. 
ground under the peak in the NMR spectra.

The cross sections and analyzing powers are compared with the results of theoretical distorted-wave impulseapproximation (DWIA) calculations in Figs. 2 and 3. The calculations by Siegel reproduce the observed IAS cross sections well. ${ }^{13}$ Cohen and Kurath (CK) wave functions ${ }^{14}$ were used for the nuclear structure. Although the results follow the trend of the analyzing powers at forward angles, the magnitudes are typically too large and the structures are too sharp. The calculations also do not describe well the excited-state cross sections and analyzing powers, although the $\frac{5}{2}^{+}$transition has not yet been included. Similar calculations by Mach with both CK and Tiator wave functions ${ }^{15}$ for the IAS transition give a better, but not perfect, description of the analyzing powers; however, the cross sections are less satisfactory. 16

The DWIA calculations of Siegel and Mach differ primarily in the detailed modeling with which the elementary pion-nucleon amplitudes are converted to the pionnucleus amplitudes $F(\theta)$ and $G(\theta)$. Both sets of calculations appear not to be strongly sensitive to the assumed model of nuclear structure, at least not to those that are limited to wave functions in the $1 p$ shell (cf., e.g., Ref. 13). Typically, the analyzing powers are less sensitive to changes of the models than are the cross sections.

The magnetic form factor for electron scattering from ${ }^{13} \mathrm{C}$, which is also sensitive to spin-dependent effects, appears to require nuclear structure wave functions that go beyond the $1 p$ shell. ${ }^{17}$ Although the momentum transfers of greatest sensitivity are a bit larger than those reached by the present data (about $1.4 \mathrm{fm}^{-1}$ ), a satisfactory description of the present analyzing-power data may require an extended model of nuclear structure. Attention should also be given to as yet unknown modifications of the elementary pion-nucleon interaction by the nuclear medium, especially its spin-dependent features.

This work was supported in part by the U.S. Department of Energy, the National Science Foundation, and the Robert A. Welch Foundation. We wish to thank the many members of the LAMPF staff for the tireless efforts that helped make this experiment possible, and the LAMPF management for their support and encouragement.

(a) Present address: EG\&G/EM, 130 Robin Hill Road, Goleta, CA 93116.

(b) Present address: Department of Physics, Old Dominion University, Norfolk, VA 23529.

(c) Present address: Department of Physics, University of New Mexico, Albuquerque, NM 87131.

(d) Present address: Paul Scherrer Institute, CH-5234 Villigen, Switzerland.

(e) Present address: Department of Physics, American University, Washington, DC 20016.

(f) Present address: Applied Research Laboratories. The University of Texas, Austin, TX 78713.

'M. E. Sevior et al., Phys. Rev. C 40, 2780 ( (1989).

${ }^{2}$ R. Tacik et al., Phys. Rev. Lett. 63, 1784 (1989).

${ }^{3}$ Y. F. Yen et al., in Proceedings of Seventh International Conference on Polarization Phenomena in Nuclear Physics, Paris, France, July 1990 (to be published).

${ }^{4}$ A. Doron et al., Phys. Rev. C 26, 189 (1982).

${ }^{5} \mathrm{H}$. W. Baer et al., Nucl. Instrum. Methods 180, 445 (1981).

${ }^{6} \mathrm{~J}$. J. Jarmer et al., Nucl. Instrum. Methods Phys. Res., Sect. A 250, 576 (1986).

${ }^{7}$ A. Abragam and M. Goldman, Nuclear Magnetism: Order and Disorder (Clarendon, Oxford, 1982).

${ }^{8}$ B. J. Dropesky et al., Phys. Rev. C 20, 1844 (1979).

${ }^{9}$ J. J. Görgen et al., Phys. Rev. D 42, 2374 (1990).

${ }^{10}$ D. Dehnhard et al., Phys. Rev. Lett. 43, 1091 (1979).

${ }^{11}$ R. J. Peterson, J. R. Shepard, and R. A. Emigh, Phys. Rev. C 24,826 (1981).

${ }^{12}$ H. Ohnuma et al., Nucl. Phys. A456, 61 (1986).

13p. B. Siegel and W. R. Gibbs, in Proceedings of the LAMPF Workshop on Physics with Polarized Nuclear Targets, edited by G. Burleson et al. (Los Alamos National Laboratory Report No. LA-10550-C, 1986); corrected calculations. P.B. Siegel (private communication); see also P. B. Siegel and W. R. Gibbs, Phys. Rev. C 36, 2473 (1987).

${ }^{14}$ S. Cohen and D. Kurath, Nucl. Phys. 73, 1 (1965).

${ }^{15}$ L. Tiator, Phys. Lett. 125B, 367 (1983).

${ }^{16} \mathrm{R}$. Mach and S. S. Kamalov, Nucl. Phys. A511. 601 (1990); R. Mach (private communication).

${ }^{17}$ R. S. Hicks et al., Phys. Rev. C 26, 339 (1982). 


\title{
Evidence for Direct Triton Knockout in the ${ }^{4} \mathrm{He}\left(\pi, \pi^{\prime} t\right) p$ Reaction
}

\author{
J. Langenbrunner, M. K. Jones, ${ }^{\left({ }^{\prime}\right)}$ and D. Dehnhard \\ Department of Physics, Unicersity of Minnesota, Minneapolis, Minnesora 55455 \\ C. L. Morris \\ Lös Alamos National Laboratory, Los Alamos, New Mexico 87545 \\ W. R. Gibbs \\ Department of Physics. New Mexico State Unicersity, Las Cruces, New Mexico 88003 \\ and Los Alamos National Laboratory. Los Alamos, New Mexico 87545
}

(Received 18 May 1992)

\begin{abstract}
Angular correlation functions for the ${ }^{4} \mathrm{He}\left(\pi^{ \pm}, \pi^{ \pm} t\right)_{p}$ reaction at the incident energy $T_{x}=180 \mathrm{MeV}$ and at four pion scattering angles have been measured. The ${ }^{4} \mathrm{He}\left(\pi^{ \pm}, \pi^{ \pm} t\right) p$ data complement previous ${ }^{4} \mathrm{He}\left(\pi^{ \pm}, \pi^{ \pm} p\right) t$. results by greatly extending the available range of proton emission angles. We calculate angular correlation functions of the cross sections with three different quasifree knockout models, and we show that direct triton knockout is crucial to an understanding of the data.
\end{abstract}

PACS numbers: $25.80 . \mathrm{Hp}, 25.10 .+\mathrm{s}, 27.10 .+\mathrm{h}$

Knockout of $\alpha$ particles from nuclei by the $\left(p, p^{\prime} \alpha\right)$ and $(\alpha, 2 \alpha)$ reactions have been studied for a wide range of targets and incident energies [1] and have provided information on the existence of $a$ clusters in the nuclear surface. Much less is known about knockout of the threenucleon clusters, ${ }^{3} \mathrm{He}$ and ${ }^{3} \mathrm{H}(t)$. In this work, we investigate triton and proton knockout from ${ }^{4} \mathrm{He}$ using $\pi^{+}$and $\pi^{-}$beams at incident energy $T_{\pi}=180 \mathrm{MeV}$, near the $P_{33}$ $[\Delta(1232)] \pi$-nucleon resonance. ${ }^{4} \mathrm{He}$ is the best possible target for a study of triton and proton knockout because approximately $90 \%$ of the total proton momentum distribution in ${ }^{4} \mathrm{He}$ can be attributed to the $p+t$ state (at small relative momentum) [2]. We show that the strong isospin dependence of the elementary $\pi$-nucleon force can be used to distinguish between direct proton knockout [3] and triton knockout, and we find that direct triton knockout is crucial to an understanding of the data.

If the ${ }^{4} \mathrm{He}\left(\pi, \pi^{\prime} p\right)$ reaction were to proceed exclusively by quasielastic $\pi$-proton scattering, we would expect a cross-section ratio $\sigma\left(\pi^{+}, \pi^{+} p\right) / \sigma\left(\pi^{-}, \pi^{-} p\right)$ equal to 9 because the ratio of $\pi^{+}$-proton to $\pi^{-}$-proton elastic scattering amplitudes is 3 for the $P_{33}$ resonance. Howev$\mathrm{er}$. if the reaction were to proceed exclusively by quasielastic $\pi$-triton scattering, we would expect a crosssection ratio $\sigma\left(\pi^{+}, \pi^{+} t\right) / \sigma\left(\pi^{-}, \pi^{-1} t\right)$ equal to $25 / 49$ $1 \approx 1 / 2)$ because the $\pi^{-}$interacts preferably with the two neutrons of the triton whereas the $\pi^{+}$interacts preferably with the one proton. Early experiments [4] studying pion-induced knockout reactions on ${ }^{4} \mathrm{He}$ at 110 and $160 \mathrm{MeV}$ used cloud chambers, but the data were not sorted according to the angle of the knocked-out nucleon. $N$. This fact limited the theoretical analysis to that of the $\left(\pi, \pi^{\prime} N\right)$ cross sections integrated over the nucleon emission angle. Calculations by Mach et al. [S] indicated that a three-nucleon exchange (knockout) amplitude is imporlant at large momentum transfer. However, the calculations failed to reproduce the ratio of total cross sections and this was interpreted as evidence for considerable initial-and/or final-state interaction effects. Tritons have been identified in the ${ }^{12} \mathrm{C}\left(\pi^{ \pm}, \pi^{ \pm \prime} t\right)^{9} \mathrm{~B}$ [6] reaction but the statistics were insufficient to warrant a firm conclusion and no attempt was made to model the reaction.

The experiment [3] was performed using the energetic pion channel and spectrometer systems (EPICS) [7] at the Clinton P. Anderson Meson Physics Facility (LAMPF). EPICS was positioned at four angles $\theta_{x}^{\text {lab }}$ $=30^{\circ}, 40^{\circ}, 60^{\circ}$, and $80^{\circ}$ on the left-hand side of the beam. In order to detect the particles emitted in coincidence with the pions, an array of plastic scintillators, each with a solid angle of $57 \mathrm{msr}$, was placed in the evacuated scattering chamber to the right-hand side of the beam at the angles $\theta^{\mathrm{lab}}=-30^{\circ},-45^{\circ},-60^{\circ},-75^{\circ}$. and $-90^{\circ}$. The experimental conditions and the analysis procedure were the same for incident $\pi^{+}$and $\pi^{-}$. Angular correlation functions for the $\pi$-proton coincidence data from a recent ${ }^{4} \mathrm{He}\left(\pi^{ \pm}, \pi^{ \pm} p\right) t$ experiment have been published by Jones et al. [3]. We have reanalyzed the raw data of that experiment and extracted $\pi$-triton coincidences.

The particle-dependent pulse height from the plastic scintillators and the particle's time of flight allowed identification and determination of the energy of the protons and tritons. In a plot of particle energy loss in the scintillators versus excitation energy $E_{x}\left({ }^{4} \mathrm{He}\right)$, the $p+t$ final state (with a separate pulse-height and time-of-fight signature for protons and tritons) was cleanly separated from particles from other reaction branches. $\theta_{p}^{\text {c.m. }}\left(\theta_{l}^{\text {c.m. }}\right)$ is the proton (triton) ejectile angle in the c.m. of the recoiling mass- 4 system. In the present work. the $\left(\pi, \pi^{\prime} t\right)$ data are plotted together with the $\left(\pi, \pi^{\prime} p\right)$ data as functions of $\theta_{p}^{\text {c.m. }}$, since $\theta_{p}^{\text {c.m. }}=\theta_{l}^{\text {c.m. }}+180^{\circ}$. Some data had been obtained in Ref. [3] due to $\pi$-proton coincidences observed in detectors placed at angles larger than $90^{\circ}$. Those data have large error bars and correspond to low- 
energy protons which were not well separated from the background. Data for the mirror reaction branch $n+{ }^{3} \mathrm{He}$ have not been extracted from this experiment because these events fell below detector threshold. A more complete description of the experiment can be found in Ref. [3].

The incoming pion of total energy and momentum $\left(E_{n}, \mathbf{k}_{\mathbf{x}}\right)$ scatters from ${ }^{4} \mathrm{He}$ such that the pion's outgoing energy and momentum are $\left(E_{x}^{\prime}, \mathbf{k}_{x}^{\prime}\right)$. Energy $\Delta T_{x}$ and momentum $q$ are transferred to the nucleus such that $\Delta T_{\pi}=E_{x}-E_{\pi}^{\prime}$ and

$$
\mathbf{q}=\mathbf{k}_{\boldsymbol{x}}-\mathbf{k}_{\boldsymbol{x}}^{\prime}=\mathbf{k}_{p}+\mathbf{k}_{\boldsymbol{t}},
$$

where $\mathbf{k}_{p}$ and $\mathbf{k}_{\text {t }}$ are the momenta of the proton and triton in the final state. The excitation energy $E_{x}$ in ${ }^{4} \mathrm{He}$ can be expressed in terms of $\Delta T_{\pi}$ and $q$ by

$$
E_{x}\left({ }^{4} \mathrm{He}\right)=\left[\left(\Delta T_{x}+m_{a}\right)^{2}-q^{2}\right]^{1 / 2}-m_{a},
$$

where $m_{a}$ is the mass of the ${ }^{4} \mathrm{He}$ nucleus.

In order to obtain the double-differential cross sections (angular correlation functions) shown in Fig. 1, we have integrated the triple-differential cross sections $d^{3} \sigma / d \Omega_{\pi}$ $\times d \Omega_{p} d E_{x}$ over the region of $E_{x}\left({ }^{4} \mathrm{He}\right)$ between 30 and $40 \mathrm{MeV}$. The condition that $E_{x}\left({ }^{4} \mathrm{He}\right)$ be greater than 30 $\mathrm{MeV}$ allowed us to identify recoiling tritons. At all pion angles, the $\pi^{+}$angular correlation data (solid dots) peak near $\theta_{p}^{\text {c.m. }}=0^{\circ}$ where proton knockout is expected to have its maximum. In sharp contrast, the $\pi^{-}$angular correlation data (open dots) show a cross section near $\theta_{p}^{\text {c.m. }}=0^{\circ}$ that is often much more than a factor of 9 smaller than for $\pi^{+}$. This observation is discussed extensively in Ref. [3]. However, the $\pi^{-}$data do show maxima near $\theta_{\rho}^{\text {c.m. }}=180^{\circ}$ because direct triton knockout is expected to generate a peak in the angular correlation. The $\pi^{+}$data also display relative maxima near $180^{\circ}$ but the cross sections for direct $\pi^{-}$-triton knockout are larger.

We have modeled the ${ }^{4} \mathrm{He}\left(\pi, \pi^{\prime} p\right) t$ reaction using the sum of the amplitudes for two processes: direct proton knockout and direct triton knockout. Angular correlation functions were calculated in this new two-amplitude quasifree (TAQ) model using the equation

$$
\frac{d^{3} \sigma}{d \Omega_{\pi} d \Omega_{p} d k_{\pi}^{\prime}}=\left|\phi\left(k_{t}\right) f_{\pi p}\left(\mathbf{k}_{\pi},-\mathbf{k}_{t}, \mathbf{k}_{\pi}^{\prime}, \mathbf{k}_{p}\right)+\phi\left(k_{p}\right) f_{\pi t}\left(\mathbf{k}_{\pi},-\mathbf{k}_{p}, \mathbf{k}_{\pi}^{\prime}, \mathbf{k}_{t}\right)\right|^{2} k_{p}^{2} \frac{d k_{p}}{d k_{\pi}^{\prime}} .
$$

The function $\phi(k)$ is a momentum-space wave function of the $p+t$ system, and for the present calculations, we use a Gaussian wave function which reproduces the charge form factor of ${ }^{4} \mathrm{He}$. The momenta in Eq. (3) are not mutually independent as can be seen from Eq. (1). In $f_{x p}$, $-k_{f}$ is the momentum of the proton before and $k_{p}$ is the momentum of the proton after the collision. In $f_{\pi t},-\mathbf{k}_{p}$ is the momentum of the triton before and $\mathbf{k}_{t}$ is the momentum of the triton after the collision. The $\pi$-proton amplitudes $f_{\pi p}$ were calculated from Ref. [8] and the $\pi$ triton amplitudes $f_{\mathrm{R} t}$ were calculated according to Ref. [9]. This model gives peak values of the cross section when $k_{t}$ or $k_{p}$ are zero; i.e., the limiting cases of Eq. (3) are two independent quasifree scatterings. This model differs from other quasifree models by including the term with $f_{x l}$ in Eq. (3). All TAQ model calculations were

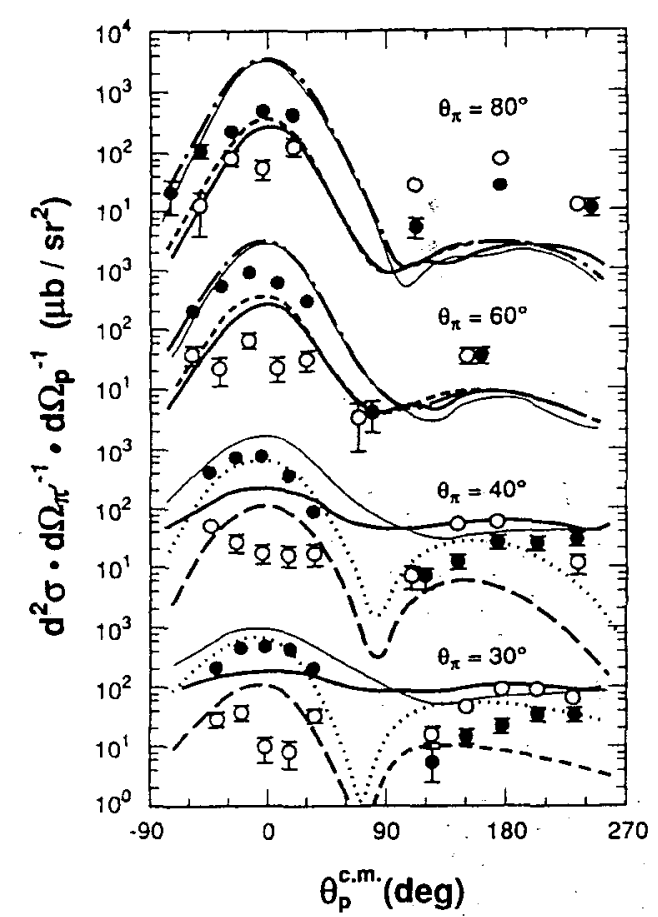

FIG. 1. Double-differential cross sections for the reactions ${ }^{4} \mathrm{He}+\pi^{+} \rightarrow p+t+\pi^{+}$(solid dots) and ${ }^{4} \mathrm{He}+\pi^{-} \rightarrow p+\imath+\pi^{-}$ (open dots) for $E_{x}$ integrated between 30 and $40 \mathrm{MeV}$. The solid curves represent our TAQ double-scattering calculations for $\pi^{+}$(thin lines) and for $\pi^{-}$(thick lines). At $\theta_{\pi}^{\text {lab }}=80^{\circ}$ and $60^{\circ}$ the dot-dashed $\left(\pi^{+}\right)$and dashed $\left(\pi^{-}\right)$curves show the TAQ single-scattering calculation. At $\theta_{k}^{\text {lab }}=40^{\circ}$ and $30^{\circ}$ the dotted $\left(\pi^{+}\right)$and long-dashed $\left(\pi^{-}\right)$curves represent the THREEDEE model calculation. Quasielastic proton scattering dominates at $\theta_{p}^{\text {c.m. }}=0^{\circ}$ whereas quasielastic triton scattering dominates at $\theta_{\rho}^{\text {c.m. }}=180^{\circ}$.

done for $E_{x}=35 \mathrm{MeV}$.

The TAQ model calculations represented by the dotdashed $\left(\pi^{+}\right)$and dashed $\left(\pi^{-}\right)$lines in Fig. 1 (only for approximation. This version of the TAQ model describes a two-body scattering problem which is being truncated, not the full scattering from four nucleons. In order to address the question of the degree of validity of the singlescattering approximation, which may not be accurate because the $\pi$-nucleon interaction is very strong, we have carried out a double-scattering TAQ calculation. The double-scattering calculations are shown as solid thin $\left(\pi^{+}\right)$and thick $\left(\pi^{-}\right)$lines in Fig. 1 (for all $\theta_{\pi}^{\text {lab }}$ ) and approximately account for a "shadowing" effect due to scattering from the spectator particle. Our present TAQ the $\theta_{\pi}^{\mathrm{lab}}=80^{\circ}$ and $60^{\circ}$ panels) assume a single-scattering 
calculations do not sufficiently describe the $\pi^{-}$-induced reaction. The inclusion of a shadowing effect does not sufficiently decrease the scattering probability to the level shown by the experiment, especially at $\theta_{p}^{\text {c.m. }}=0^{\circ}$. At this point, our calculation with double scattering should be taken only as an indication of the size of the effect. For example, for $\pi^{-}$scattering to $\theta_{\pi}^{\text {lab }}=80^{\circ}$ and $\theta_{p}^{\text {c.m. }}=0^{\circ}$, the effect is to decrease the cross section by roughly $20 \%$.

Calculations from the one-nucleon knockout model THREEDEE [10] are represented by dotted $\left(\pi^{+}\right)$and long-dashed lines $\left(\pi^{-}\right.$) in Fig. 1 (only for $\theta_{\pi}^{\text {lab }}=30^{\circ}$ and $40^{\circ}$ ) and compared to the TAQ double-scattering predictions (solid lines). The THREEDEE calculations are made in the framework of the impulse approximation. They include (using a factorization approximation) the distortions of the incoming pion and target-nucleus system, the outgoing pion and residual-nucleus system, and the knocked-out proton and residual-nucleus system. The THREEDEE predictions for double-differential cross sections depend strongly on various distortions [3]. The back-angle rise in the THREEDEE predictions near $\theta_{r^{\prime}}^{\mathrm{c} \cdot \mathrm{m} .}=180^{\circ}$ is a consequence of distortions, not of triton knockout. These calculations do not include a $\pi$-triton amplitude $f_{\pi t}$ as in Eq. (3). The THREEDEE calculations at back angles are an order of magnitude smaller than the data for $\pi^{-}$.

One can estimate the effect of the momentum distribution on quasifree scattering cross sections by using the ansatz that the recoil momentum of the spectator particle $\left(k_{\text {spec }}\right)$ is equal to its momentum before scattering. We calculated excitation energies where a $p+t$ momenturn distribution has its maximum value $\left(k_{\text {spec }}=0\right)$ and halfmaximum value [2] $\left(k_{\text {spec }}=0.45 \mathrm{fm}^{-1} \approx 90 \mathrm{MeV} / c\right)$. The values of $E_{x}$ for $k_{\text {spec }}=0$ are listed in Table $I$ in the columns denoted by $E_{x}^{\text {q.f. }}$. Deviations from $E_{x}^{\text {q.f. }}$ for $E_{x}$ such that $k_{\text {spec }}=90 \mathrm{MeV} / \mathrm{c}$ are indicated by the superscripts and subscripts in Table I. For example, at $\theta_{\pi}^{\text {lab }}=40^{\circ}$, quasifree proton knockout occurs with maximal probability when $E_{x}=34 \mathrm{MeV}$. With $E_{x}=33 \mathrm{MeV}$, direct triton knockout occurs with roughly one-half of the maximal probability because the (spectator) proton is

TABLE I. The predicted centroids of quasifree peaks in the excitation energy spectra are given for all pion scattering angles in columns labeled $E_{x}^{\text {q.f. }}\left(\pi, \pi^{\prime} p\right)$ and $E_{x}^{\text {q.f. }}\left(\pi, \pi^{\prime} t\right)$. For these values, the spectator particle is emitted with zero momentum $\left(k_{\text {spec }}=0\right)$. The superscripts and subscripts give, if added to $E_{x}^{\text {y.f. }}$, the excitation energies corresponding to $k_{\text {spec }} \approx 90 \mathrm{MeV} / c$, as discussed in the text. Subscripts are omitted when breakup is energetically forbidden. The momentum transfers $q$ are given for $E_{x}=35 \mathrm{MeV}$.

\begin{tabular}{cccc}
\hline \hline$\theta_{x}^{\text {lab }}(\mathrm{deg})$ & $q\left(\mathrm{fm}^{-1}\right)$ & $E_{x}^{\text {q.f. }}\left(\pi, \pi^{\prime} p\right)(\mathrm{MeV})$ & $E_{x}^{\text {q.f. }}\left(\pi, \pi^{\prime} \ell\right)(\mathrm{MeV})$ \\
\hline 30 & 0.73 & $28^{+18}$ & $22^{+9}$ \\
40 & 0.94 & $34 \pm 18$ & $22^{+11}$ \\
60 & 1.35 & $47^{+32}$ & $23^{+11}$ \\
80 & 1.70 & $60 \pm 25$ & $26^{+12}$ \\
\hline \hline
\end{tabular}

emitted with $\approx 90 \mathrm{MeV} / c$. Also given in the table is the momentum transfer $q$ for each of the pion scattering angles with $E_{x}=35 \mathrm{MeV}$.

We define an isospin ( $T)$ asymmetry $A_{T}\left(\theta_{p}^{\mathrm{cm}}\right)$. in analogy with the spin observable $A_{\eta}$, with the equation

$$
A_{T}\left(\theta_{P}^{\text {c.m. }}\right)=\frac{\sigma\left(\pi^{+}, \pi^{+\prime} X\right)-\sigma\left(\pi^{-}, \pi^{-\prime} X\right)}{\sigma\left(\pi^{+}, \pi^{+\prime} X\right)+\sigma\left(\pi^{-}, \pi^{-\prime} X\right)},
$$

where $X=p$ or $t$. In the plane-wave impulse approximation (PWIA) and for $P_{33}$ resonance dominance, the observable $A_{T}\left(\theta_{p}^{\text {c.m. }}\right)$ is isotropic and equal to +0.8 for pure quasielastic proton knockout. For pure quasielastic triton knockout, we expect $A_{T}\left(\theta_{p}^{\text {c.m. }}\right)$ to be isotropic and equal to $\approx-0.3$. These results are independent of the scattering angle of the pion and proton. Moreover, if the pion scattering excites ${ }^{4} \mathrm{He}$ to a resonance state with pure isospin, its decay is governed exclusively by the properties of that state, and we expect $A_{T}=0$. Thus, exclusive, kinematically complete $\pi^{+}$and $\pi^{-}$scattering should easily distinguish between proton knockout, triton knockout, or resonance formation and decay for these reasons: the prediction of isotropy in $A_{T}\left(\theta_{p}^{\text {c.m. }}\right)$ in the

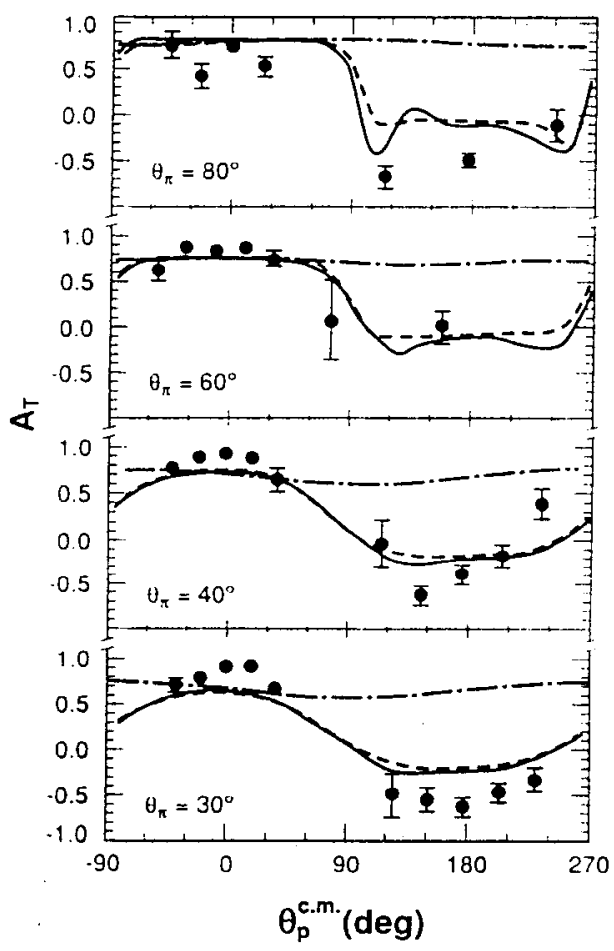

FIG.'2. Isospin asymmetry $A_{T}$ ( $\left.\theta_{p}^{\text {c.m. }}\right)$ [Eq. (4)] for the reactions ${ }^{4} \mathrm{He}+\pi^{ \pm} \rightarrow p+1+\pi^{ \pm}$, for $E_{x}$ integrated between 30 and $40 \mathrm{MeV}$. The data show. large isospin asymmetries, changing from +1.0 near $\theta_{p}^{\text {c.m. }}=0^{\circ}$ to -0.6 near $\theta_{\rho}^{\text {c.m. }}=180^{\circ}$. The solid and dashed lines of the TAQ model qualitatively describe the data with and without double scattering and show the importance of direct triton knockout near $\theta_{p}^{\text {c.m. }}=180^{\circ}$. The dotdashed lines represent proton knockout calculations (with distortions) using THREEDEE [10]. These asymmetries are essentially isotropic, because that model does not include a $\pi$-triton amplitude such as $f_{s t}$ in Eq. (3). 
case that a single amplitude is involved in the scattering, and the large values and different signs of asymmetry for quasifree proton and triton scattering.

The data for $A_{T}{ }^{\left(\theta_{p}^{\text {c.m. }}\right)}$ in Fig. 2 vary strongly with $\theta_{p}^{\text {c.m. }}$ from near +1.0 in the kinematic region of quasifree proton knockout $\left(\theta_{\rho}^{\text {c.m. }}=0^{\circ}\right)$, to about -0.6 in the kinematic region of quasifree triton knockout $\left(\theta_{p}^{\text {c.m. }}=180^{\circ}\right)$. This result requires that at least two amplitudes contribute to the reaction. The TAQ model with the $\pi$-triton scattering amplitude successfully describes the sign reversal of $A_{T}\left(\theta_{\rho}^{\text {c.m. }}\right)$ with (solid lines) or without the shadowing effect (dashed lines). The prediction using THREEDEE (dash-dotted lines) with only a $\pi$-proton interaction is essentially isotropic (near the PWIA prediction of $A_{T}$ $=+0.8$ ). This is our strongest evidence for direct triton knockout.

Use of different pion-nucleus optical potentials and proton-triton optical potentials in THREEDEE can affect the calculations of cross sections [3] by factors of 2 to 3; however, these changes yield values of $A_{T}\left(\theta_{p}^{\text {c.m. }}\right)$. which differ by less than $2 \%$. The THREEDEE model calculations deviate slightly from $A_{T}\left(\theta_{p}^{\text {c.m. }}\right)=+0.8$ because of distortions and/or contributions from the $s$-wave $\pi$ nucleon interaction. The dependence of $A_{T}\left(\theta_{p}^{\text {c.m. }}\right)$ on variations of the TAQ model is also small, because shadowing effects in the TAQ model are similar for $\pi^{-}$- and $\pi^{+}$-induced reactions.

While there is ample evidence from $A_{T}\left(\theta_{p}^{\text {c.m. }}\right)$ that triton knockout is present, the TAQ model underestimates the magnitude of $A_{T}$ near $\theta_{p}^{\text {c.m. }}=0^{\circ}$ and $180^{\circ}$ (Fig. 2). The TAQ description of the $\pi^{-}$-proton data near the quasifree proton angle is poor and the TAQ calculation of the $\pi^{+}$-proton cross section (Fig. 1) varies with proton angle more slowly than the data. The TAQ model may be improved by use of ${ }^{4} \mathrm{He}$ wave functions such as in Ref. [2]. The largest variation between the model calculations is near $\theta_{p}^{\text {c.m. }}=90^{\circ}$ and $-90^{\circ}$, where interference effects in the TAQ model are maximized, but where there are currently no data.

Clearly, additional physics issues need to be considered. Of these, nuclear resonance states may be important, but based on a recent compilation and study [11], we expect that we avoid much of the complicated nuclear structure of ${ }^{4} \mathrm{He}$ below $E_{x} \approx 30 \mathrm{MeV}$. Another possibility is a charge-exchange $(n, p)$ process which is expected to be particularly strong near $\theta_{p}^{c . m .}=0^{\circ}$ for $\pi^{-}$. The two-step charge-exchange reaction mechanism $\left(e, e^{\prime} n\right)$ followed by $(n, p)$ was studied in a model of the exclusive reaction [12] ${ }^{4} \mathrm{He}\left(e, e^{\prime} p\right)$. Calculations that combined the effects of $(n, p)$ charge exchange, meson exchange, and proton rescattering showed that an $(n, p)$ charge-exchange process increases the ${ }^{4} \mathrm{He}\left(e, e^{\prime} p\right)$ cross section by about $10 \%$ [12]. Direct triton knockout was not included in that cal- culation.

Kyle et al. [13] have modeled the ${ }^{16} \mathrm{O}\left(\pi^{ \pm}, \pi^{ \pm \prime} p\right)$ reaction by including a $\Delta-N$ interaction. In that model a $\Delta$ induced proton knockout amplitude interferes with the quasifree proton knockout process. Understanding of the present data for ${ }^{4} \mathrm{He}$ may improve with the application of such a model, but that model has not been extended yet to include a $\pi$-triton interaction.

We conclude that the isospin dependence of the $\pi$ triton interaction in the region of the $P_{3,3}$ resonance can be used to identify direct triton knockout from the nucleus. Experiments with $\pi^{+}$and $\pi^{-}$on other nuclei in which tritons (and possible ${ }^{3} \mathrm{He}$ ) are detected could provide evidence for the occurrence of preexisting clusters of mass 3. Exclusive scattering experiments with $\pi^{+}$and $\pi^{-}$on light nuclei should allow us to refine reaction models and nuclear wave functions. For the ${ }^{4} \mathrm{He}\left(\pi^{ \pm}, \pi^{ \pm \prime} t\right) p$ reaction, the direct $\pi$-triton scattering amplitude appears to be an essential component of the reaction mechanism.

This work was supported in part by the U.S. Department of Energy.

${ }^{(a)}$ Present address: Rutgers University, Piscataway, NJ 08855.

[1] D. F. Jackson, in Clustering Phenomena in Nuclei, edited by $\mathrm{P}$. Kramer and $\mathrm{K}$. Wildermuth (Vieweg-Verlag, Wiesbaden, 1983), Vol. 3.

[2] R. Schiavilla, V. R. Pandharipande, and R. B. Wiringa, Nucl. Phys. A449, 219 (1986).

[3] M. K. Jones et al., Phys. Rev. C 46, 52 (1992); 42, R807 (1990)

[4] F. Balestra et al., Nuovo Cimento 15, 542 (1976): Nuovo Cimento Lett. 41, 391 (1984).

[5] R. Mach et al., Nuovo Cimento Soc. Ital. Fis. 45A, 325 (1978).

[6] R. J. Ellis et al., Phys. Rev. C 26, 1544 (1982); Phys. Lett. 88B, 253 (1979).

[7] H. A. Thiessen and S. Sobottka, LASL Report No. LA4534-MS, 1978 (unpublished).

[8] G. Rowe, M. Salomon, and R. H. Landau, Phys. Rev. C 18, 584 (1978).

[9] W. R. Gibbs and B. F. Gibson, Phys. Rev. C 43, 1012 (1991).

[10] N. S. Chant, computer code THREEDEE (private communication); N. S. Chant, L. Rees, and P. G. Roos, Phys. Rev. Lett. 48, 1784 (1982); L. Rees, N. S. Chant, and P. G. Roos, Phys. Rev. C 26, 1580 (1982).

[11] D. R. Tilley, H. R. Weller, and G. M. Hale, Nucl. Phys. A541, 1 (1992).

[12] J. F. J. van den Brand et al., Phys. Rev. Lett. 66, 409 (1991).

[13] G. S. Kyle et al., Phys. Rev. Lett. 52, 974 (1984) 


\title{
Asymmetries for Elastic Scattering of $\pi^{+}$from Polarized ${ }^{3} \mathrm{He}$ and the $\Delta$-Neutron Spin-Spin Interaction
}

M. A. Espy, D. Dehnhard, C. M. Edwards, ${ }^{*}$ M. Palarczyk, J. L. Langenbrunner, and B. Davis School of Physics and Astronomy; University of Minnesota, Minneapolis, Minnesota 55455

G. R. Burleson, S. Blanchard, W. R. Gibbs, B. Lail, B. Nelson. B. K. Park,* and Q. Zhao Department of Physics, New Mexico State University, Las Cruces, New Mexico 88003

W. J. Cummings,,$^{\ddagger}$ P. P. J. Delheij, B. K. Jennings, R. Henderson, O. Häusser, and D. Thiessen TRIUMF, Vancouver, Canada V6T $2 A 3$

E. Brash and M. K. Jones

Rutgers University, Piscataway, New Jersey 08854

B. Larson ${ }^{k}$

Department of Physics and Astronomy, Ohio University, Athens, Ohio 45701

B. Brinkmöller"I

Physikalisches Institut der Universität, Karlsruhe, Germany

K. Maeda

Department of Physics, Tohoku University, Kawauchi, Sendai, Japan

C. L. Morris, J. M. O'Donnell, ${ }^{k}$ S. Penttilä, D. Swenson, ${ }^{* *}$ and D. Tupa Los Alamos National Laboratory, Los Alamos, New Mexico 87545

C. Bennhold

Center for Nuclear Studies, Department of Physics, The George Washington University, Washington, D.C. 20052

S. S. Kamalov

JINR Dubna, Moscow, Russia

(Received 22 November 1995)

\begin{abstract}
Asymmetries for $\pi^{+}$elastic scattering from polarized ${ }^{3} \mathrm{He}$ were measured across the $\Delta(1232) \pi$ nucleon resonance region at incident energies $T_{\pi}=142,180$, and $256 \mathrm{MeV}$. Large discrepancies were found between the data and calculations that use the multiple scatrering formalism and Faddeev walve functions. Inclusion of a $\Delta$-neutron spin-spin interaction term in the calculations gives a greatly improved representation of the data. [S0031-9007(96)00229-3]
\end{abstract}

PACS numbers: 25.80.Dj, 13.75.Cs. 24.70.+s, 25.10.+s

In this Letter we report on the first measurement of asymmetries $A_{y}$, for $\pi^{+}$elastic scattering from polarized ${ }^{3} \mathrm{He}$ at incident energies which pass through the $\Delta(1232)$ $\pi$-nucleon resonance. We find evidence for the importance of the $\Delta(1232)$-neutron spin-spin interaction in the nucleus. Knowledge of the strength of the spin-dependent (and spin-independent) parts of the $\Delta$-nucleon interaction is needed for tests of meson-exchange [1] and quark models [2] of the baryon-baryon interaction.

Measurements on polarized ${ }^{3} \mathrm{He}$ recently became possible with the development of the high-density, optically pumped ${ }^{3} \mathrm{He}$ gas target at TRIUMF [3]. This target was used in a $\pi^{+}$scattering experiment and very large values of $A_{1}$, were found at $T_{\pi}=100 \mathrm{MeV}$ [4].

These large values of $A_{y}$ are in contrast with the generally small values obtained from scattering on $p$ - shell nuclei of $\operatorname{spin} 1 / 2[5-8]$. The theoretically [9-11] unexpected small $A_{y}$ for ${ }^{15} \mathrm{~N}$ and ${ }^{13} \mathrm{C}$ imply shortcomings in our present understanding of either the $\pi$-nucleus interaction and specitically its spin-dependent part. or of the nuclear wave functions of these $p$-shell nuclei. or both. For the much simpler nucleus ${ }^{3} \mathrm{He}$. reliable wave functions have been obtained by Faddeev calculations. $[12,13]$. Thus spin-dependent effects in the $\pi$-nucleus interaction can be studied without large uncertainties in the nuclear structure. However, at $100 \mathrm{MeV}$ the $A_{y}$ for ${ }^{3} \mathrm{He}$ show only a slight dependence on the reaction model.

Thus measurements of $\pi^{+}$elastic scattering from polarized ${ }^{3} \mathrm{He}$ at energies near the centroid and above the $\Delta(1232)$ resonance. where $A_{y}$ is predicted $|14.15|$ to be very sensitive to the detaits of the reaction model. are of great interest for a study of the spin-dependent parts of the 
pion-nucleus interaction. We note that sensitivity to the small components of the ${ }^{3} \mathrm{He}$ wave function is predicted at $T_{\pi} \geq 256 \mathrm{MeV}$.

The TRIUMF target was set up in the $\mathrm{P}^{3} \mathrm{E}$ area at LAMPF, where high beam fluxes were available across the region of the $\Delta(1232)$ resonance. The scattered pions were detected with the large acceptance spectrometer (LAS) [16]. Measurements were made at incident energies $T_{\pi}=142,180$, and $256 \mathrm{MeV}$ and laboratory scattering angles ranging from $40^{\circ}$ to $100^{\circ}$. Some data were taken at $100 \mathrm{MeV}$ and found to be in agreement with the results of Ref. [4].

The ${ }^{3} \mathrm{He}$ gas was contained in a cylindrical glass cell, ahout $4.8 \mathrm{~cm}$ in diameter and $6.5 \mathrm{~cm}$ in length. Target cells were made of quarty glass that was about $1.5 \mathrm{~mm}$ thick at the cylindrical cell walls and $0.4 \mathrm{~mm}$ thick at the hemispherical endcaps (where the pion beam entered and exited the cell). The cells were filled with 6-7 atm of ${ }^{3} \mathrm{He}$ gas, a trace of $\mathrm{Rb}$, and a small amount of $\mathrm{N}_{2}$ which served as a buffer gas. $8-10 \mathrm{~W}$ of polarized laser light at $795 \mathrm{~nm}$ (the $D 1$ transition in $\mathrm{Rb}$ ) from two argon pumped Ti:sapphire lasers and solid state diode laser arrays were used to polarize the $\mathrm{Rb}$ atoms in the target cell. The electron spin polarization of the $\mathrm{Rb}$ was transferred through $\mathrm{Rb}-{ }^{3} \mathrm{He}$ collisions to the ${ }^{3} \mathrm{He}$ nucleus hy the contact hyperfine interaction. The target cell was heated continuously in the target oven to a temperature of approximalely $175^{\circ} \mathrm{C}$ in order to achieve the required $\mathrm{Rb}$ vapor density for the optical pumping. When the glass cell was hot, small amounts of ${ }^{3} \mathrm{He}$ leaked from the cell. Therefore, the pressure in the cell and the cell temperatures were monitored periodically so that a correction for the pressure loss could be made.

The target apparatus was modified during the experiment by the addition of a diode laser array [17]. The diode laser added to the optical pumping power and significantly increased the polarization after one of the argon lasers failed. ${ }^{3} \mathrm{He}$ polarization was typically $35 \%$ to $45 \%$, sometimes reaching $50 \%$. Since the helicity of the laser light determines the direction of the target polarization, the orientation of the ${ }^{3} \mathrm{He}$ spins (and thus the sign of $A_{y}$ ) was determined by use of a liquid crystal which transmits only left-hand circularly polarized light. One set of Helmholtz coils provided a vertical holding field for the polarization. Another set of Helmholtz coils provided a variable horizontal field component employed for changing the direction of the polarization. The magnitude of the polarization was measured using the nuclear magnetic resonance (NMR) technique of adiabatic fast passage (AFP) [3]. Absolute normalization factors for the NMR signals were obtained by comparing the NMR signals from the ${ }^{3} \mathrm{He}$ with the weak signals from the protons in a water-filled cell of the same dimension.

The LAS uses a magnetic quadrupole doublet, a magnetic dipole, scintillation detectors, and several sets of two-dimensional wire chambers in order to identify the scattered pions and to measure their momenta [16]. The front wire chambers allow traceback of the scattered particle trajectories to a plane that intersects the center of the target perpendicular to the central ray of the LAS. The projections of the reaction vertices onto this plane were used to discriminate between events from ${ }^{3} \mathrm{He}$ and the glass in the end caps and the top and bottom of the cylindrical cell wall. Events from the left and right sides of the cylindrical wall could not be eliminated by software cuts. Thus the beam halo striking the sides of the cell was reduced by a lead collimator that was machined to match the beam divergence. In order to enable subtraction of the remaining background, spectra were taken at many angles with an evacuated target.

The top and center panels of Fig. 1 show yields as a function of the negative $Q$ value of the reaction measured at $180 \mathrm{MeV}$ and $\theta_{\mathrm{lab}}=50^{\circ}$, normalized to the integrated beam flux for the two target spin orientations. The target polarization parallel to the norm of the reaction plane is indicated by $\uparrow$, the one antiparallel by $\downarrow$. The ${ }^{3} \mathrm{He}$ elastic peak, centered at $Q=0$, has a width of about $4 \mathrm{MeV}$ (FWHM). The difference spectrum is shown in the bottom panel of Fig. 1. A large negative $A_{y}$ is apparent in the region of the elastic peak from ${ }^{3} \mathrm{He}$.

The experimental $A_{y}$ was obtained using

$$
A_{y}=\frac{N_{\uparrow}-N_{l}}{N_{1}+N_{l}} \frac{1}{p} \text {. }
$$

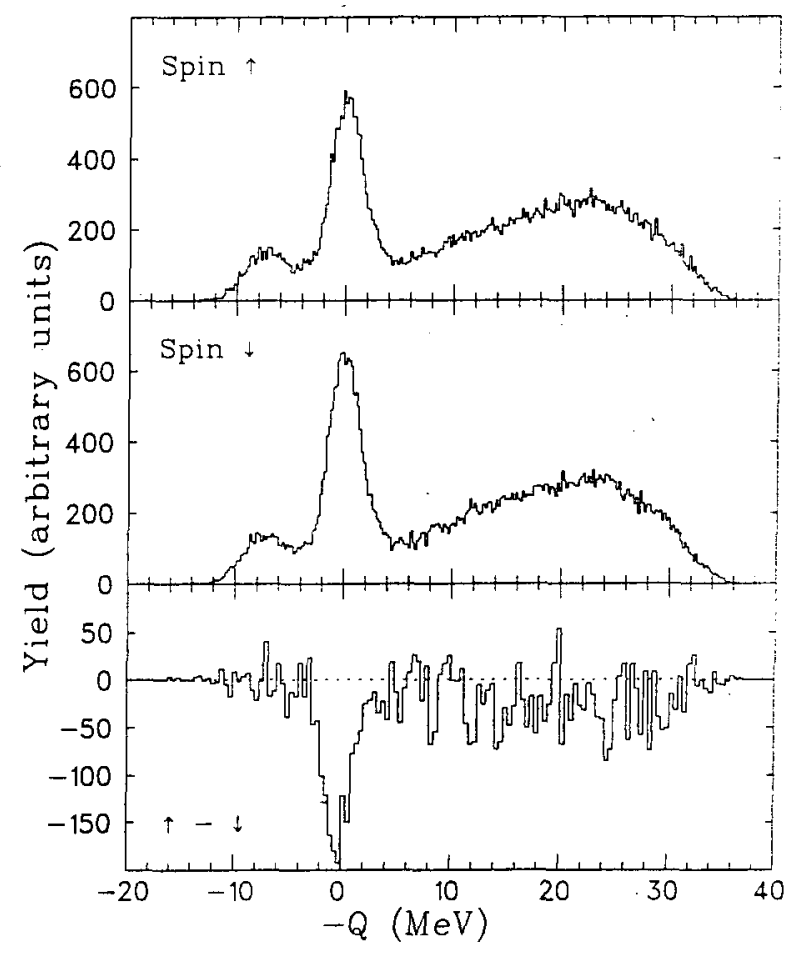

FIG. 1. Typical normalized energy spectra from elastic scattering of $\pi^{+}$on the polarized ${ }^{3} \mathrm{He}$ target at $T_{\pi}=180 \mathrm{MeV}$ and $\theta_{\text {lab }}=50^{\circ}$. Top panel: target spin parallel $(\uparrow)$; center panel: target spin antiparallel $(\downarrow)$ to the normal to the reaction plane. The difference spectrum is shown in the bottom panel. 
Here $N_{\uparrow}$ and $N_{\downarrow}$ are the normalized numbers of counts in the ${ }^{3} \mathrm{He}$ elastic peak with the ${ }^{3} \mathrm{He}$ spins oriented "up" and "down," respectively. $p$ is the target polarization. Background events cancel in the difference $N_{\uparrow}-N_{\downarrow}$ and were subtracted before taking the sum. The theoretical $A_{y}$ in pion scattering from a spin $1 / 2$ nucleus can be written in terms of the complex spin-independent $(F)$ and spindependent $(G)$ scattering amplitudes as

$$
A_{y}=\frac{2 \operatorname{Im}\left(F G^{*}\right)}{\left(|F|^{2}+|G|^{2}\right)} \text {. }
$$

Experimental and theoretical angular distributions of $A_{y}$ for $\pi^{+}$elastic scattering at $T_{\pi}=142,180$, and $256 \mathrm{MeV}$ are presented in Fig. 2. The solid lines were obtained using multiple scattering theory and three-body Faddeev wave functions $[12,13]$ for ${ }^{3} \mathrm{He}$ to calculate the first-order terms in $F$ and $G$. The dashed lines employ a hybrid model (see below) that uses the first-order values of $F$ and $G$ but includes a second-order contribution to $G$ from the $\Delta$-neutron spin-spin interaction calculated in the plane wave impulse approximation (PWIA).

Neither the conventional multiple scattering calculations of Ref. [15] (Fig. 2, solid lines) nor those of one of us (W. R. G.) and of Ref. [18] (not shown) give a satisfactory description of $A_{y}$ at 142 and $180 \mathrm{MeV}$. At both energies we observe large positive $A_{y}$ near $80^{\circ}$ as predicted by the calculation, but the maximum of $A_{y}$ is shifted towards larger angles. At scattering angles near $60^{\circ}$ the measured

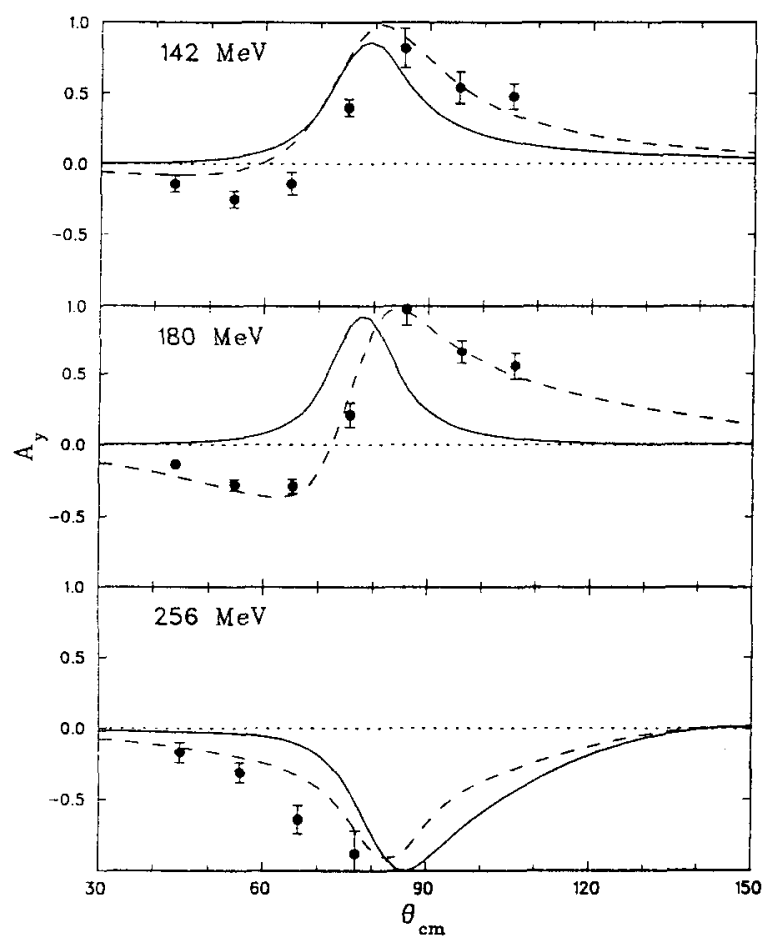

FIG. 2. Asymmetry angular distribution for elastic $\pi^{+}$. scattering from polarized ${ }^{3} \mathrm{He}$ at $T_{\pi}=142 \mathrm{MeV}$ (top), $180 \mathrm{MeV}$ (center), and $256 \mathrm{MeV}$ (bottom). Solid lines: Multiple scattering predictions of Ref. [15] using Faddeev wave functions. Dashed lines: Predictions of the hybrid model (see text).
$A_{y}$ are negative at 142 and $180 \mathrm{MeV}$, in contradiction to any of the conventional model calculations which predict positive $A_{y}$ between 100 and $180 \mathrm{MeV}$ at these angles. Use of a simple $s$-state wave function for ${ }^{3} \mathrm{He}$ and the PWIA predicts positive $A_{y}$ at all angles [14] (not shown) similar to the calculations using Faddeev wave functions (solid lines) at 142 and $180 \mathrm{MeV}$. At $256 \mathrm{MeV}$ this simple model predicts positive $A_{y}$ at all angles, whereas the multiple scattering calculations give negative $A_{y}$. The negative $A_{y}$ at $256 \mathrm{MeV}$ (but not at 142 and $180 \mathrm{MeV}$ ) can also be obtained by modifying the real part of $F$ in a way that accounts for multiple scattering [14]. Except in the minimum, the differential cross sections at 142 and $180 \mathrm{MeV}$ (not shown) are fit quite well with the multiple scattering calculations. We note that a preliminary calculation that uses a purely scalar phenomenological $\rho^{2}$ term in the optical potential to account for pion absorption [19] predicts $A_{y}$ completely out of phase with the data at $180 \mathrm{MeV}$

Large second-order elfects may be ciatsed by the 1 neutron interaction when the $\Delta(1232)$ resonance dominates the elementary $\pi$-nucleon interaction. For this resonance the isospin coupling Clebsch-Gordan coefficients result in much larger scattering amplitudes $F$ and $G$ for $\pi^{+}$elastic scattering on protons than on neutrons. But the $\pi^{+}$interacting with the paired-off protons in a $(1 s)^{3}$ ground state of ${ }^{3} \mathrm{He}$ cannot contribute to the first-order spin-dependent amplitude $G$ which results only from scattering from the unpaired neutron. $F$ has a large (first-order) component from scattering from the two protons and a small one from scattering from the neutron. A large second-order contribution to $G$ arises if the intermediate $\Delta^{++}$. generated with very high probability in $\pi^{+}$scattering on one of the two protons, interacts with the polarized neutron.

The magnitude of this second-order contribution to $G$ has been investigated by one of us (B. J.) using the simple $s$-shell model for ${ }^{3} \mathrm{He}$ with Gaussian single particle wave functions. The $\mathrm{rms}$ radius of the nucleon distribution in ${ }^{3} \mathrm{He}$ was kept fixed at the value $\langle r\rangle^{1 / 2}=1.65 \mathrm{fm}$ obtained by unfolding the finite proton size from the charge, density of ${ }^{3} \mathrm{He}[20]$. Furthermore, the model employs the PWIA for this second-order term and a meson exchange model for the $\Delta$-neutron interaction which includes the $\pi, \rho, \omega$, and $\eta$ mesons. The mesun$\Delta$ couplings were obtained from the meson-nucleon couplings by use of SU(6) symmetry and the naive quark model. Two-nucleon correlations were included phenomenologically by multiplying the wave functionwith a Gaussian correlation function that depends on the relative distance of the interacting particles. The width of the correlation Gaussian was kept fixed at a standard value of $0.75 \mathrm{fm}[21]$. The resulting second-order term in $G$ was added to the first-order multiple scattering values for $F$ and $G$.

This hybrid model gives a very good tit to $A_{y}$ at $180 \mathrm{MeV}$ (dashed line in Fig. 2). The negative $A_{y}$ near $60^{\circ}$ and the shift of the positive maximum towards larger 
angles are reproduced. At $142 \mathrm{MeV}$ the magnitude of the negative $A_{y}$ near $60^{\circ}$ is not described as well. At $256 \mathrm{MeV}$ the model including the $\Delta$-neutron interaction is in slightly better agreement with the data than the multiple scattering calculation without the $\Delta$-neutron interaction (solid line). The effect of this interaction on the differential cross section is to fill in the minimum at 142 and $180 \mathrm{MeV}$, resulting in an improved fit (not shown).

Further theoretical work is needed to determine whether our measured $A_{y}$ can be explained by some aspect of the reaction mechanism or the ${ }^{3} \mathrm{He}$ wave function, which we have not yet included. Effects from pion absorption need to be studied further by, for example, including spin and isospin parts in the second-order corrections to the optical potential ( $\rho^{2}$ term). The $\Delta$-neutron interaction should be treated as part of the full multiple scattering calculations and not simply added on as in this work. At $256 \mathrm{MeV}$ the small components in the ${ }^{3} \mathrm{He}$ wave function have to be included [15]. No predictions of $A_{y}$ for ${ }^{3} \mathrm{He}$ are currently available within the framework of the $\Delta$-hole model. Previous work (reviewed in Ref. [22]) invoked the need of a strong but purely phenomenological $\Delta$-nucleus spinorbit potential. A microscopic derivation [23] of this term gave a much smaller strength. Nevertheless, its effect on the $A_{y}$, should be considered in future calculations. Our hybrid model is the first attempt to treat the $\Delta$-nucleon interaction in $\pi^{+3} \mathrm{He}$ scattering microscopically.

On the experimental side, data for $\pi^{-}$scattering on polarized ${ }^{3} \mathrm{He}$ are needed. For $\pi^{-}$scattering the firstorder contribution to $G$ is much larger than the secondorder contribution so that the effect from the $\Delta$-nucleon interaction on $A_{v}$ is predicted to be negligible.

In conclusion, asymmetries were measured for $\pi^{+}$ elastic scattering from polarized ${ }^{3} \mathrm{He}$ at energies near the pion-nucleon $\Delta(1232)$ resonance. Thus far conventional pion-nucleus interaction theory has failed to fit the data. However, a large second-order contribution to the spindependent scattering amplitude in $\pi^{+}{ }^{3} \mathrm{He}$ scattering, resulting from the $\Delta$-neutron spin-spin interaction, significantly improves the agreement between experiment and theory.

The authors wish to thank Dr. R. L. Boudrie for his unrelenting support and the technical staff at LAMPF for assistance. This work was supported by the U.S. Department of Energy and the National Science Foundation.

*Present address: Los Alamos National Laboratory, Los Alamos. NM 87545.
${ }^{\dagger}$ Present address: Ohio University, Athens, $\mathrm{OH} 45701$.

tPresent address: Argonne National Laboratory, Argonne. IL 60439 .

"Present address: College of William \& Mary, Williamsburg, VA 23187.

kPresent address: University of Minnesota, Minneapolis, MN 55455.

TPresent address: S.A.P.-A.G., Karisruhe, Germany.

**Present address: Varian Ion Implant Systems, Gloucester, MA 01930.

[1] R. Machleidt, Adv. Nucl. Phys, 19, 189 (1989).

[2] M. Johnson and L. Kisslinger, Phys. Rev. C 52, 1022 (1995).

[3] B. Larson et al., Phys. Rev. A 44, 3108 (1991).

[4] B. Larson et al., Phys. Rev. Lett. 67, 3356 (1991): B. Larson et al, Phys. Rev. C 49, 2045 (1994).

[5] R. Tacik et al., Phys. Rev. Lett. 63, 1784 (1989).

[6] Yi-Fen Yen et cl., Phys. Rev. Lett. 66, 1959 (1991).

[7] J. T. Brack et al., Phys. Rev. C 45, 698 (1992).

[8] Yi-Fen Yen, Ph.D. thesis, University of Minnesota, 1991; Yi-Fen Yen et al., Phys. Rev. C 50, 897 (1994).

[9] R. Mach and S.S. Kamalov, Nucl. Phys. A511, 601 (1990).

[10] S. Chakravarti et al., University of Minnesota, Annual Report 1990 (unpublished); D. Dehnhard et al., Few-Body Syst. Suppl. 5, 274 (1992).

[11] P. B. Siegel and W. R. Gibbs, Phys. Rev. C 48, 1939 (1993).

[12] R. A. Brandenburg, Y.E. Kim, and A. Tubis. Phys. Rev. C 12, 1368 (1975).

[13] J. L. Friar et al., Phys. Rev. C 34, 1463 (1986).

[14] C. Bennhold, B. K. Jennings, L. Tiator, and S. S. Kamalov, Nucl. Phys. A540, 621 (1992).

[15] S. S. Kamalov, L. Tiator, and C. Bennhold, Phys. Rev. C 47, 941 (1993).

[16] A. L. Williams. Ph.D. thesis, University of Texas at Austin, 1991; Los Alamos National Laboratory Report No. LA-12209-T, 199l.

[17] TRIUMF Annual Report, 1993 (unpublished); W. J. Cummings, O. Häusser, W. Lorenzon, D. R. Swenson, and B. Larson, Phys. Rev. A 51. 4842 (1995).

[18] S. Chakravarti, C. M. Edwards, D. Dehnhard. and M.A. Franey, Few-Body Syst. Suppl. 5, 267 (1992).

[19] M. Gmitro, S. S. Kamalov, and R. Mach, Phys. Rev. C 36. 1105 (1987).

[20] H. De Vries, C. W. De Jager, and C. De Vries, At. Nucl. Data Tables 36, 495 (1987).

[21] B. H. J. McKellar and B. F. Gibson, Phys. Rev. C 30, 322 (1984).

[22] T. Ericson and W. Weise, Pions and Nuclei (Clarendon Press, Oxford, 1988), pp. 237-251.

[23] T.-S. Lee and K. Ohta, Phys. Rev. C 25, 3043 (1982). 